\begin{tabular}{|c|l|}
\hline Title & Die Eigenschwingungen und Klangfiguren der vierseitig freien rechteckigen Platte \\
\hline Author(s) & Iguchi, Shikazo \\
\hline Citation & Memoirs of the Faculty of Engineering, Hokkaido Imperial University, 6(5), 317-385 \\
\hline Issue Date & 1942-04_30 \\
\hline Doc URL & http://hdl.handle.net/2115/37736 \\
\hline Type & bulletin (article) \\
\hline File Information & 6(5)_317-386.pdf \\
\hline
\end{tabular}

Instructions for use 


\title{
Die Eigenschwingungen und Klangfiguren der vierseitig freien rechteckigen Platte.
}

\author{
Von
}

Shikazo IGUchI.

(Eingegangen am. 25. Okt. 1941).

\section{INHALTSVERZEICHNIS.}

Einleitung $\cdots \cdots \cdots \cdots \cdots \cdots$

I. Kapitel. Allgemeine Erörterung . . . . . . . . . . . . . . . . . . 319

1. Die Grundgleichung . . . . . . . . . . . . . . . . . 319

2. Allgemeine Lösung und die Randbedingungen ... . . . . . . . . . . . 319

II. Kapitel. Die Platte mit Eckstützen . . . . . . . . . . . . . . . . 321

A. Allgemeines . . . . . . . . . . . . . . . . 321

3. Die allgemeinen Formeln. . . . . . . . . . . . . . 321

4. Die Symmetrie- und Antisymmetrieeigenschaft der Schwingungstläche . . . 323

B. Die erste Schwingungsart . . . . . . . . . . . . . . 323

5. Die allgemeinen Formeln fïr die rechteckige Platte . . . . . . . . 323

6. Die Formeln und die numerischen Rechenergebnisse für die quadratische Platte. 326

C. Die zweite Schwingungsart . . . . . . . . . . . 335

7. Die allgemeinen Formeln für die rechteckige Platte . . . . . . . 335

8. Die Formeln und die numerischen Rechenergebnisse für die quadratische Platte. 338

D. Die dritte Schwingungsart . . . . . . . . . . . . . . . 341

9. Die allgemeinen Formeln fïr die rechteckige Platte . . . . . . . . 341

10. Die Formeln und die numerischen Rechenergebnisse für die quadratische Platte. 343

III. Kapitel. Die Platte ohne Eckstitze . . . . . . . . . . . . . . . . . . 348

A. Allgemeines . . . . . . . . . . . . . . . . . . 348

11. Die allgemeinen Formeln . . . . . . . . . . . . . 348

B. Die erste Schwingungsart. . . . . . . . . . . . . 350

12. Die allgemeinen Formeln für die rechteckige Platte . . . . . . . . 350

13. Die Formeln und die numerischen Rechenergebnisse für die quadratische Platte. 353

14. Näherungsformeln für die quadratische Platte . . . . . . . . . 361

c. Die zweite schwingungsart . . . . . . . . . . . . . . . . . . 362

15. Die allgemeinen Formeln für die rechteckige Platte . . . . . . . . . 362

16. Die Formeln und die numerischen Rechenergebnisse für die quadratische Platte. 364

D. Die dritte Schwingungsart. . . . . . . . . . . . . . . . 371

17. Die allgemeinen Formeln fïr die rechteckige Platte . . . . . . . . . 371

18. Die Formeln und die numerischen Rechenergebnisse für die quadratische Platte. 372

E. Experimentelle Untersuchung . . . . . . . . . . . . . . 383 


\section{EINLEITUNG.}

Die Eigenschwingungserscheinungen der elastischen quadratischen Platte mit freien Rändern sind schon seit Anfang des letzten Jahrhunderts durch die von E. F. F. Chladni(1) experimentell hergestellten Sandfiguren (Klangfiguren) wohlbekannt. Seit jener Zeit haben zahlreiche Forscher, nämlich Ch. Wheatstone,2) F. Strehlle, ${ }^{3)}$ R. König,,4) S. 'Tanaka,5) Lord Rayleigh' ${ }^{6)}$ u. a. das genannte Problem entweder experimentell oder analytisch untersucht und bedeutende und interessante Leistungen auf dem Gebiet der Akustik und Elastizitätslehre geschaffen. Was aber die theoretischen Untersuchungen betrifft, so sind sie alle wegen der Schwierigkeiten der Randbedingungen der Platte nur Näherungslösungen.

Im Beginn dieses Jahrhunderts ist die wertvolle Arbeit, von W. Ritz? erschienen, in welcher er das Problem unter Benutzung der Eigenfunktionen für den geraden Stab mit freien Enden mittels der Energiemethode gelöst hat. Insbesondere ist bemerkenswert, dass seine aus seinen Formeln angefertigten Klangfiguren mil nur wenigen Abweichungen im Einklang mit den Chladnischen. Figuren stehen. Trotzdem ist dies auch eine Näherungslösung, denn seine Formeln genügen nicht vollständig den Randbedingungen der Platte. So blieb es bis jetzt unerreichtet, das betreffende Problem streng zu lösen.

Im folgenden suche ich die Lösungen zu geben, welche sowohl die Differentialgleichung als auch die Plattenrandbedingungen vollständig befriedigen. Die Randbedingungen der allseitig freien rechteckigen Platte, ob sie in ihren vier Eckpunkten gestützt wird oder nicht, werden immer durch dieselben mathematischen Ausdrücke definiert, nur mit dem Unterschied, dass die zu der zweiten Ableitung $\frac{\partial^{2} w}{\partial x \partial y}$ proportionalen Einzelkräfte in jeder Ecke auftreten oder nicht. So handelt es sich im folgenden zunächst um die Platte, mit Eckstützen und dann weiter um die Platte ohne Eckstütze, namlich die Platte mit vollkommen freien Rändern.

Die Schwingungsflächen werden nach ihren Gestalten in drei Arten (die erste, zweite und dritte Schwingungsart) klassifiziert. Als Anwendungsbeispiel meiner Formeln werden die den verschiedenen Tonhöhen jeder Schwingungsart entsprechenden 9 Eigenwerte $(\mu)$ für die Platte mit Eckstützen und 23 Eigenwerte für die Platte ohne Eckstütze ausgerechnet. Dazu noch sind die daraus hervorgehenden 13 Knotenfiguren nebst den Schichtlinien der Schwingsungsfläche für die Platte mit Eckstützen angegeben. Was die Platte ohne Eckstütze betrifft, mit der wir uns hauptsächlich beschäftigen wollen, so werden die den 23 Eigenwerten angehörenden 35 Knotenfiguren nebst den entsprechenden Schichtlinien der Schwingungsfläche ermittelt, welche mit den ebenso von mir als auch von Chladni experimentell gebildeten Sandfiguren hinlänglich gut übereinstimmen.

1) Chladni, "Die Akustik, Leipzig" 1802; Neudruck 1830.

2) Ch. Wheatstone, Phil. Trans. 1833.

3) F. Strehlke, Pogg. Ann. 95 (1855); 146 (1872).

4) R. König, Pogg. Ann. 122 (1864).

5) S. 'Tanaka, Ann. Phys. 32 (1887).

6) Lord Rayleigh, "Therry of Sound," 1 S. 372, 1929.

7) W. Ritz, Ann. Phys. 28 (1909) \$. 737. 


\section{Kapitel.}

\section{ALLGEMEINE ERO̊RTERUNG.}

1. Die Grundgleichung. Ist $w$ die Auslenkung zur Zeit $t$ an der Stelle $(x, y)$ der Mittelebene einer homogenen dünnen Platte, dann gilt für die Biegungsschwingungen die Differentialgleichung

$$
\frac{\partial^{4} w}{\partial x^{4}}+2 \frac{\partial^{4} w}{\partial x^{2} \partial y^{2}}+\frac{\partial^{4} w}{\partial y^{4}}+\frac{\rho h}{N} \frac{\partial^{2} w}{\partial t^{2}}=0
$$

Hierin bedeutet $\rho$ die Plattendichte und $N$ die Plattensteifigkeit, d. h. $E h^{3} / 12$ $\left(1-\nu^{2}\right)$, wenn $h$ die Plattendicke, $E$ der Elastizitätsmodul und $\nu$ die Querkontraktionszahl des Stoffes ist.

Bezeichnet man nun mit $w^{\prime}$ eine nur von den Veränderlichen $x$ und $y$ abhängige Funktion, also die Eigenfunktion, und mit $\omega$ die Kreisfrequenz der Eigenschwingungen, so wird $w$ durch das Produkt

$$
w=w^{\prime} \cos \omega t
$$

ausgedrückt. Setzt man (2) in (1) ein. so erhält man

$$
\frac{\partial^{4} w^{\prime}}{\partial x^{4}}+2 \frac{\partial^{4} w^{\prime}}{\partial x^{2} \partial y^{2}}+\frac{\partial^{4} w^{\prime}}{\partial y^{4}}-\frac{\omega^{2} \rho h}{N} w^{\prime}=0
$$

woraus man die Funktion $w^{\prime}$ zu finden hat.

2. Die allgemeine Lösung und die Randbedingungen. Es handelt sich jetzt um die Aufösung der Differentialgleichung (3). $\mathrm{Zu}$ diesem Zwecke nimmt man zunächst für die Platte mit den Seitenlängen $a$ und $b$, wie Abb. 1 zeigt, an

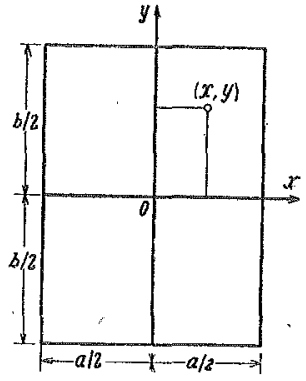

Abb. 1. Schema der Koordinatenachsen.

$$
w^{\prime}=X_{n}\left(\frac{x}{a}\right) \sin n \pi\left(\frac{1}{2}+\frac{y}{b}\right) \quad \text { oder } \quad w^{\prime}=X_{n}\left(\frac{x}{a}\right) \cos n \pi\left(\frac{1}{2}+\frac{y}{b}\right) .
$$

Die Einführung dieses Ansatzes für $w^{\prime}$ in (3) liefert die charakteristische Gleichung

$$
\left[D^{2}-\left(\frac{a^{2}}{b^{2}} n^{2} \pi^{2}+\omega a^{2} \sqrt{\frac{\rho h}{N}}\right)\right]\left[D^{2}-\left(\frac{a^{2}}{b^{2}} n^{2} \pi^{2}-\omega a^{2} \sqrt{\frac{\rho h}{N}}\right)\right]=0, \quad\left(D \equiv \frac{d}{d x}\right)
$$

aus der die vier Wurzeln

$$
D= \pm \sqrt{\frac{a^{2}}{b^{2}} n^{2} \pi^{2}+\omega a^{2} \sqrt{\frac{\rho h}{N}}}, \quad \pm \sqrt{\frac{a^{2}}{b^{2}} n^{2} \pi^{2}-\omega a^{2} \sqrt{\frac{\rho h}{N}}}
$$

folgen. Damit und mit den Abkürzungen 


$$
\begin{aligned}
& \lambda_{\alpha_{n}}=\sqrt{\alpha^{2} n^{2}+\mu}, \quad \lambda_{\alpha_{n}}^{\prime}=\sqrt{\alpha^{2} n^{2}-\mu}, \quad\left(a \equiv \frac{a}{b}\right) \\
& \mu=\frac{\omega a^{2}}{\pi^{2}} \sqrt{\frac{\rho h}{N}} \quad \text { (dimensionslose Grösse) }
\end{aligned}
$$

ergibt sich

$$
X_{n}\left(\frac{x}{a}\right)=e^{\frac{\pi \lambda_{a n}}{a} x}, e^{-\frac{\pi \lambda_{\alpha}}{a} x}, e^{\frac{\pi \lambda_{\alpha_{n}}^{\prime}}{a} x}, e^{-\frac{\pi \lambda_{a}^{\prime}}{a} x}
$$

oder auch

$$
X_{n}\left(\frac{x}{a}\right)=\operatorname{Cof} \frac{\pi \lambda_{\alpha_{n}}}{a} x, \quad \operatorname{Sin} \frac{\pi \lambda_{\alpha_{n}}}{a} x, \quad \operatorname{Cof} \frac{\pi \lambda_{\alpha_{n}}^{\prime}}{a} x, \quad \operatorname{Sin} \frac{\pi \lambda_{\varkappa_{n}}^{\prime}}{a} x
$$

Aus dem analogen Ansatz

$$
w^{\prime}=Y_{m}\left(\frac{y}{b}\right) \sin m \pi\left(\frac{1}{2}+\frac{x}{a}\right) \quad \text { oder } \quad w^{\prime}=Y_{m}\left(\frac{y}{b}\right) \cos m \pi\left(\frac{1}{2}+\frac{x}{a}\right)
$$

erhält man

$$
Y_{m}\left(\frac{y}{b}\right)=\operatorname{Cof} \frac{\pi \lambda_{\beta m}}{b} y, \quad \operatorname{Sin} \frac{\pi \lambda_{\beta m}}{b} y, \quad \operatorname{Cof} \frac{\pi \lambda_{\beta m}^{\prime}}{b} y, \quad \operatorname{Sin} \frac{\pi \lambda_{3 m}^{\prime}}{b} y
$$

mit den Abkürzungen

$$
\left.\begin{array}{l}
\lambda_{\beta m}=\sqrt{\beta^{2} m^{2}+\mu^{\prime}}, \quad \lambda_{\beta m}^{\prime}=\sqrt{\beta^{2} m^{2}-\mu^{\prime}}, \quad\left(\beta \equiv \frac{b}{a}\right) \\
\mu^{\prime}=\frac{b^{2}}{a^{2}} \mu=\frac{\omega b^{2}}{\pi^{2}} \sqrt{\frac{\rho h}{N}} . \quad \text { (dimensionslose Grösse) }
\end{array}\right\}
$$

Somit schreibt sich nun die allgemeine Lösung von (3), wie folgt:

$$
\left.\begin{array}{c}
w^{\prime}=\sum_{n} X_{n}(\xi)\left\{\begin{array} { l } 
{ \operatorname { s i n } n \pi ( \frac { 1 } { 2 } + \eta ) } \\
{ \operatorname { c o s } n \pi ( \frac { 1 } { 2 } + \eta ) }
\end{array} \sum _ { m } Y _ { m } ( \eta ) \left\{\begin{array}{l}
\sin m \pi\left(\frac{1}{2}+\xi\right) \\
\cos m \pi\left(\frac{1}{2}+\xi\right)
\end{array}\right.\right. \\
\left(m, n=\left\{\begin{array}{ll}
1,2,3 \ldots \text { für die Sinusreihen } \\
0,1,2 \ldots & \text { für die Cosinusreihen }
\end{array}\right),\right. \\
\xi \equiv \frac{x}{a}, \quad \eta \equiv \frac{y}{b}
\end{array}\right\}
$$

mit

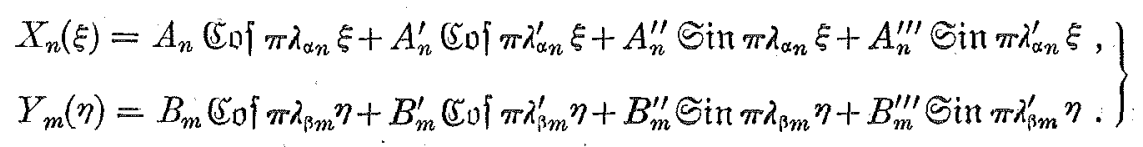


Für die durch Abb. 1 dargestellte rechteckige Platte gelten die Randbedingungen
1) $\frac{\partial^{2} w^{\prime}}{\partial x^{2}}+\nu \frac{\partial^{2} w^{\prime}}{\partial y^{2}}=0$
für $\quad x= \pm \frac{a}{2}$,
2) $\frac{\partial^{2} w^{\prime}}{\partial y^{2}}+\nu \frac{\partial^{2} w^{\prime}}{\partial x^{2}}=0$
für $y= \pm \frac{b}{2}$,
3) $\frac{\partial}{\partial x}\left\{\frac{\partial^{2} w^{\prime}}{\partial x^{2}}+(2-\nu) \frac{\partial^{2} w^{\prime}}{\partial y^{2}}\right\}=0$
für $x= \pm \frac{\alpha}{2}$,
4) $\frac{\partial}{\partial y}\left\{\frac{\partial^{2} w^{\prime}}{\partial y^{2}}+(2-\nu) \frac{\partial^{2} w^{\prime}}{\partial x^{2}}\right\}=0$
fǜr $\quad y= \pm \frac{b}{2}$.

Die acht Integrationskonstanten in $X_{n}(\xi)$ und $\mathrm{Y}_{m}(\eta)$ sind miteinander so $\mathrm{zu}$ verknüpfen, dass die Funktion $w^{\prime}(6 a)$ mit $(6 b)$ den vorangehenden Randbedingungen genügt.

Man muss hierbei noch beachten, dass die Auslenkung $w^{\prime}$ in den vier Ecken versohwindet, wenn dje Platte in ihren Eckpunkten unbeweglich (mit Eckstützen) gehalten wird; ersichtlich gilt dafür $w^{\prime}(6 a)$ mit Sinusreihen. Wenn aber alle Plattenränder vollkommen frei sind (ohne Eckstützen), so muss der Differentialquotient $\frac{\partial^{2} w^{\prime}}{\partial x \partial y}$ in den vier Ecken verschwinden, weil in den freien Ecken keine konzentrierte Kiaft auftreten kann. Man sieht aber leicht, dass $w^{\prime}(6 a)$ mit Cosipusreihen von vornherein diesen Eckenbedingungen genügt.

\section{Kapitel.}

\section{DIE PLATTE MIT ECKSTÜTZEN.}

\section{A. Allgemeines.}

3. Die allgemeinen Formeln. Wie in der vorangehenden Ziff., erwähnt, gilt für die Platte mit Eckstützen

$$
\begin{gathered}
w^{\prime}=\sum_{n} X_{n}(\xi) \sin n \pi\left(\frac{1}{2}+\eta\right)+\sum_{m} Y_{m}(\eta) \sin m m\left(\frac{1}{2}+\xi\right) . \\
(m, n=1,2,3 \ldots)
\end{gathered}
$$

Da die Kreisfunktion $\sin m \pi\left(\frac{1}{2}+\xi\right)$ selbst wie auch ihre zweite Ableitung für die Werte $\xi= \pm \frac{1}{2}$ verschwindet, so geht aus den Randbedingungen 1) eine einfache Beziehung

$$
\frac{a^{2}}{\pi^{2}} X_{n}^{\prime \prime}\left( \pm \frac{1}{2}\right)-\nu \alpha^{2} n^{2} X_{n}\left( \pm \frac{1}{2}\right)=0
$$


hervor. Daraus und aus der ersten Formel (6b) folgt

$$
\begin{aligned}
& A_{n}\left(\lambda_{\alpha n}^{2}-\nu \alpha^{2} n^{2}\right) \operatorname{Cof} \frac{\pi}{2} \lambda_{\alpha_{n}}+A_{n}^{\prime}\left(\lambda_{\alpha n}^{\prime 2}-\nu \alpha^{2} n^{2}\right) \operatorname{cof} \frac{\pi}{2} \lambda_{\alpha n}^{\prime} \\
& \pm\left\{A_{n}^{\prime \prime}\left(\lambda_{\alpha n}^{2}-\nu \alpha^{2} n^{2}\right) \operatorname{Sin} \frac{\pi}{2} \lambda_{\alpha_{n}}+A_{n}^{\prime \prime \prime}\left(\lambda_{\alpha n}^{2}-\nu \alpha^{2} n^{2}\right) \operatorname{Sin} \frac{\pi}{2} \lambda_{\alpha_{n}}^{\prime}\right\}=0 \text {. }
\end{aligned}
$$

Die Summe bzw. die Differenz der obigen beiden ${ }^{\circ}$ Gleichungen liefert die Beziehung

$$
A_{n}\left(\lambda_{\alpha n}^{2}-\nu \alpha^{2} n^{2}\right) \operatorname{Coj} \frac{\pi}{2} \lambda_{\alpha n}+A_{n}^{\prime}\left(\lambda_{\alpha n}^{\prime 2}-\nu \alpha^{2} n^{2}\right) \mathfrak{C o j} \frac{\pi}{2} \lambda_{\alpha_{n}}^{\prime}=0
$$

bzW.

$$
A_{n}^{\prime \prime}\left(\lambda_{\alpha n}^{2}-\nu \alpha^{2} n^{2}\right) \Subset i n \frac{\pi}{2} \lambda_{\alpha n}+A_{n}^{\prime \prime \prime}\left(\lambda_{\alpha n}^{\prime 2}-\nu \alpha^{2} n^{2}\right) \operatorname{Sin} \frac{\pi}{2} \lambda_{\alpha n}^{\prime}=0
$$

Damit und mit den neu eingeführten Beiwerten $a_{n}$ und $a_{n}^{\prime}$ ergibt sich

$$
\begin{array}{ll}
A_{n}=a_{n} \frac{\lambda_{\alpha n}^{\prime 2}-\nu \alpha^{2} n^{2}}{\operatorname{cof} \frac{\pi}{2} \lambda_{\alpha n}}, & A_{n}^{\prime}=-a_{n} \frac{\lambda_{\alpha n}^{2}-v \alpha^{2} n^{2}}{\operatorname{Co} f \frac{\pi}{2} \lambda_{\alpha n}^{\prime}}, \\
A_{n}^{\prime \prime}=a_{n}^{\prime} \frac{\lambda_{\alpha n}^{\prime \prime}-\nu \alpha^{2} n^{2}}{\operatorname{Sin} \frac{\pi}{2} \lambda_{\alpha n}}, & A_{n}^{\prime \prime \prime}=-a_{n}^{\prime} \frac{\lambda_{\alpha n}^{2}-\nu \alpha^{2} n^{2}}{\operatorname{Sin} \frac{\pi}{2} \lambda_{\alpha n}^{\prime}} .
\end{array}
$$

Die Funktion $X_{n}(\xi)$ lässt sich jetzt schreiben, wie folgt:

$$
X_{n}(\xi)=a_{n} u_{\alpha_{n}}(\xi)+a_{n}^{\prime} v_{\alpha_{n}}(\xi) \quad(n=1,2,3 \ldots)
$$

mit

$$
\left.\begin{array}{l}
u_{\alpha_{n}}(\xi)=\left(\lambda_{\alpha_{n}}^{2}-\nu \alpha^{2} n^{2}\right) \frac{\operatorname{So}\left[\pi \lambda_{\alpha_{n}} \xi\right.}{\operatorname{Soj} \frac{\pi}{2} \lambda_{\alpha_{n}}}-\left(\lambda_{\alpha_{n}}^{2}-\nu \alpha^{2} n^{2}\right) \frac{\operatorname{Soj}^{2} \lambda_{\alpha_{n} \xi}^{\prime} \xi}{\operatorname{Soj} \frac{\pi}{2} \lambda_{\alpha_{n}}^{\prime}}, \\
v_{\alpha_{n}}(\xi)=\left(\lambda_{\alpha_{n}}^{\prime 2}-\nu \alpha^{2} n^{2}\right) \frac{\operatorname{Sin} \pi \lambda_{\alpha_{n}} \xi}{\operatorname{Sin} \frac{\pi}{2} \lambda_{\alpha_{n}}}-\left(\lambda_{\alpha_{n}}^{2}-\nu \alpha^{2} n^{2}\right) \frac{\operatorname{Sin} \pi \lambda_{\alpha_{n}}^{\prime} \xi}{\operatorname{Sin} \frac{\pi}{2} \lambda_{\alpha_{n}}^{\prime}}
\end{array}\right\}
$$

In analoger Weise erhält man aus den Randbedingungen 2)

$$
Y_{m}(\eta)=b_{m} u_{\beta m}(\eta)+b_{m}^{\prime} v_{\beta m}(\eta) \quad(m=1,2,3 \ldots)
$$

mit

$$
\left.\begin{array}{l}
u_{\beta m}(\eta)=\left(\lambda_{\beta m}^{\prime 2}-\nu \beta^{2} m^{2}\right) \frac{\operatorname{Cof} \pi \lambda_{\beta m} \eta}{\operatorname{Sof} \frac{\pi}{2} \lambda_{\beta m}}-\left(\lambda_{\beta m}^{2}-\nu \beta^{2} m^{2}\right) \frac{\operatorname{Cof} \pi \lambda_{i m}^{\prime} \eta}{\operatorname{Gof} \frac{\pi}{2} \lambda_{\beta m}^{\prime}}, \\
v_{\beta m}(\eta)=\left(\lambda_{\beta m}^{\prime 2}-\nu \beta^{2} m^{2}\right) \frac{\operatorname{Sint} \pi \lambda_{\beta m} \eta}{\operatorname{Sin} \frac{\pi}{2} \lambda_{\beta m}}-\left(\lambda_{\beta m}^{2}-\nu \beta^{2} m^{2}\right) \frac{\operatorname{Sin} \pi \lambda_{\beta m}^{\prime} \eta}{\operatorname{Sin} \frac{\pi}{2} \lambda_{\beta m}^{\prime}}
\end{array}\right\}
$$


Die in (8) und (9) noch verfügbaren unbekannten Beiwerte $a_{n}, a_{n}^{\prime}, b_{m}, b_{m}^{\prime}$ müssen miteinander so verknüpft werden, class $w^{\prime}(7)$ mit (8) und (9) den übrigen Randbedingungen 3) und 4) genügen. Da aber die diesbezüglichen weiteren Rechnungen viel komplizierter sind, als die für die Randbedingungen 1) und 2), so werden sie in den kommenden Einzelfällen durchgeführt.

4. Die Symmetrie- und Antisymmetrieeigenschaft der Schwingungsfläche. Fs ist leicht ersichtlich, dass

$$
u_{\alpha n}(-\xi)=u_{\alpha_{n}}(\xi), \quad v_{\alpha_{n}}(-\xi)=-v_{\alpha_{n}}(\xi)
$$

ist. Ferner wird

$$
\begin{aligned}
\sin m \pi\left(\frac{1}{2}+\xi\right) & =\sin \frac{m \pi}{2} \cos m \pi \xi+\cos \frac{m \pi}{2} \sin m \pi \xi \\
& =(-1)^{\frac{m-1}{2}} \cos m \pi \xi \quad \text { für } m=1,3,5 \ldots \\
& =(-1)^{\frac{m}{2}} \sin m \pi \xi \quad \text { für } m=2,4,6 \ldots
\end{aligned}
$$

Somit versteht man, dass neben dem Verschwinden der Beiwerte $a_{n}^{\prime}$ bzw. $a_{n}$ die Zeiger $m$ in (7) bis (9) die ungeraden bzw. geraden Zahlen durchlaufen, wenn die Schwingungsfläche $w^{\prime}(7)$ sich symmetrisch bzw. antisymmetrisch in bezug auf die Mittellinie $\xi=0$ gestaltet. Analog müssen die Beiwerte $b_{m}^{\prime} b z w . b_{m}$ verschwinden, und gleichzeitig die Zeiger $n$ die ungeraden bzw. geraden Zahlen nehmen, je nachdem die Platte symmetrisch bzw. antisymmetrisch in bezug auf die Mittellinie $\eta=0$ schwingt. Die beiden. Beiwerte $a_{n}$ und $a_{n}^{\prime}$ oder $b_{m}$ und $b_{m}^{\prime}$ können daher nur dann gleichzeitig auftreten, wenn die Schwingungsfläche $w^{\prime}$ unsymmetrisch in der $x$ - oder $y$-Richtung ist.

\section{B. Die erste Schwingungsart.}

5. Die allgemeinen Formeln für die rechteckige Platte. Wir wollen uns erstens allgemein mit der ersten Schwingungsart beschäftigen, in. welcher die Schwingungsfläche symmetrisch in bezug auf die beiden Mittellinien $\xi=0$ und $\eta=0$ des Rechtecks ist. Wegen dieser Symmetrieeigenschaft der Schwingungsfäche schreibt sich die Funktion $w^{\prime}(7)$, wie folgt:

$$
\begin{gathered}
w^{\prime}=\sum_{n}(-1)^{\frac{n-1}{2}} a_{n} u_{\alpha n}(\xi) \cos n \pi \eta+\sum_{m}(-1)^{\frac{m-1}{2}} b_{m} u_{\beta m}(\eta) \cos m \pi \xi . \\
(m, n=1,3,5 \ldots)
\end{gathered}
$$

Durch Einführung dieses Ausdrucks für $w^{\prime}$ in die Randbedingungen 3) erhält man

$$
\begin{aligned}
& \sum_{n}(-1)^{\frac{n-1}{2}} a_{n}\left\{\frac{a^{3}}{\pi^{3}} u_{\alpha n}^{\prime \prime \prime}\left( \pm \frac{1}{2}\right)-\alpha^{2} n^{2}(2-\nu) \frac{a}{\pi} u_{\alpha n}^{\prime}\left( \pm \frac{1}{2}\right)\right\} \cos n \pi \eta \\
& \quad \pm \sum_{m} b_{m}\left\{m^{3} u_{\beta m}(\eta)-\alpha^{2} m(2-\nu) \frac{b^{2}}{\pi^{2}} u_{\beta m}^{\prime \prime}(\eta)\right\}=0
\end{aligned}
$$


worin lautet

$$
\begin{aligned}
& \frac{a^{3}}{\pi^{3}} u_{\alpha n}^{\prime \prime \prime}\left( \pm \frac{1}{2}\right)-a^{2} n^{2}(2-\nu) \frac{a}{\pi} u_{\alpha n}^{\prime}\left( \pm \frac{1}{2}\right)=\mp G_{\alpha_{n}} \text { (abkürzungsweise), } \\
& = \pm \lambda_{\alpha_{n}}^{2}\left(\lambda_{\alpha_{n}}^{2}-\nu \alpha^{2} \pi^{2}\right) \mathfrak{I} \mathfrak{g} \frac{\pi}{2} \mp \lambda_{\alpha_{n}}^{\prime 2}\left(\lambda_{\alpha_{n}}^{2}-\nu \alpha^{2} n^{2}\right) \mathfrak{I} \mathfrak{g} \frac{\pi}{2} \lambda_{\alpha_{n}}^{\prime} \\
& \mathcal{F} \alpha^{2} n^{2}(2-\nu)\left\{\lambda_{\alpha_{n}}\left(\lambda_{\alpha_{n}}^{\prime 2}-\nu \alpha^{2} n^{2}\right) \mathfrak{I g} \frac{\pi}{2} \lambda_{\alpha_{n}}-\lambda_{\alpha_{n}}^{\prime}\left(\lambda_{\alpha_{n}}^{2}-\nu \alpha^{2} n^{2}\right) \mathfrak{T g} \frac{\pi}{2} \lambda_{\alpha_{n}}^{\prime}\right\} \text {, } \\
& =\mp\left\{\lambda_{\alpha n}\left(\lambda_{\alpha n}^{\prime 2}-\nu \alpha^{2} \lambda^{2}\right)^{2} \mathfrak{I} \mathfrak{g} \frac{\pi}{2}-\lambda_{\alpha n}^{\prime}\left(\lambda_{\alpha n}^{2}-\nu^{2} \alpha^{2} n^{2}\right)^{2} \mathfrak{I} \mathfrak{g} \frac{\pi}{2} \lambda_{\alpha n}^{\prime}\right\} ; \\
& m^{3} u_{\beta m}(\eta)-\alpha^{2} m(2-\nu) \frac{b^{2}}{\pi^{2}} u_{\beta m}^{\prime \prime}(\eta)=U_{\beta m}(\eta) \quad \text { (abkürzungsweise) } \\
& =m^{3}\left(\frac{\lambda_{\beta m}^{2} \subseteq 0\left\lceil\pi \lambda_{\beta m} \eta\right.}{\operatorname{Cof} \frac{\pi}{2} \lambda_{\beta m}}-\frac{\lambda_{\beta m}^{2} \subseteq 0\left\lceil\pi \lambda_{\beta m}^{\prime} \eta\right.}{\operatorname{Cof} \frac{\pi}{2} \lambda_{\beta m}^{\prime}}\right) \\
& -\left\{\nu \beta^{2} m^{5}+\alpha^{2} m(2-\nu) \lambda_{\beta m}^{2} \lambda_{\beta m}^{\prime 2}\right\}\left(\frac{\complement_{0}\left\{\pi \lambda_{\beta m} \eta\right.}{\complement_{0}\left\lceil\frac{\pi}{2} \lambda_{\beta m}\right.}-\frac{\mathfrak{C O}_{0} \pi \lambda_{\beta m}^{\prime} \eta}{\operatorname{Cof} \frac{\pi}{2} \lambda_{\beta m}^{\prime}}\right)
\end{aligned}
$$

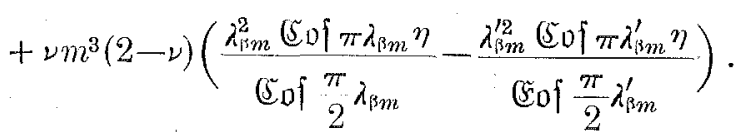

Vermöge der Formeln ${ }^{1)}$

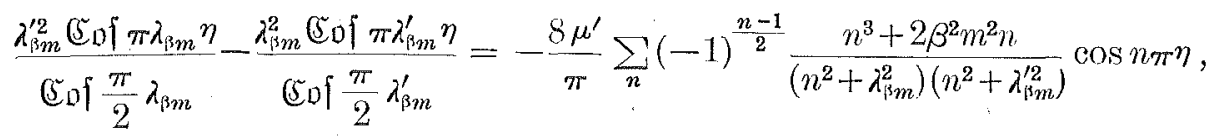

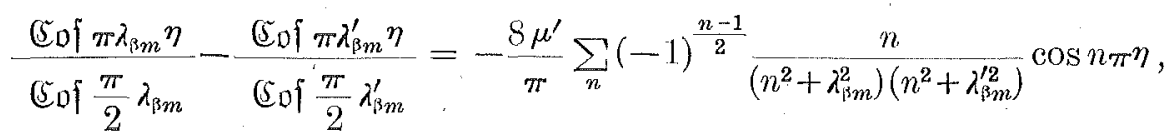

$$
\begin{aligned}
& \frac{\lambda_{\beta m}^{2} \mathfrak{C O}_{0} \pi \lambda_{\beta m} \eta}{\mathfrak{C O}_{0} \frac{\pi}{2} \lambda_{\beta m}}-\frac{\lambda_{B m}^{\prime 2} \mathfrak{C O}_{0} \pi \lambda_{\beta m}^{\prime} \eta}{\operatorname{Cof} \frac{\pi}{2} \lambda_{\beta m}^{\prime}}=\frac{8 \mu^{\prime}}{\pi} \sum_{n}(-1)^{\frac{n-1}{2}} \frac{n^{3}}{\left(n^{2}+\lambda_{\beta m}^{2}\right)\left(n^{2}+\lambda_{\beta m}^{\prime 2}\right)} \cos n \pi \eta \\
& \left(-\frac{1}{2}<\eta<\frac{1}{2}, \quad n=1,3,5 \ldots\right)
\end{aligned}
$$

kann man die Funktion $U_{\beta m}(\eta)$ in Fourietsche Reihe nach $\cos n \pi \eta$ entwickeln, wie folgt:

$$
U_{\beta m}(\eta)=-\frac{8 \mu^{\prime}}{\pi} \sum_{n}(-1)^{\frac{n-1}{2}} m n \frac{(1-\nu)^{2} m^{2} n^{2}+(2-\nu) \alpha^{2} \mu^{\prime 2}}{\left(n^{2}+\lambda_{s m}^{2}\right)\left(n^{2}+\lambda_{\beta m}^{\prime 2}\right)} \cos n \pi \eta .
$$

1) S. Iguchi, "Eine Lösung für die Berechnung der biegsamen rechteckigen Platte," S. 15 bis 19, Berlin 1933 . 
Gemäss (b) und (c) geht die vorhergehende Gleichung (a) über in

$$
\sum_{n}(-1)^{\frac{n-1}{2}}\left[a_{n} G_{\alpha_{n}}+\frac{8 \mu^{\prime}}{\pi} \sum_{m} b_{m} \frac{m n\left\{(1-\nu)^{2} m^{2} n^{2}+(2-\nu) \alpha^{2} \mu^{\prime 2}\right\}}{\left(n^{2}+\lambda_{\beta m}^{2}\right)\left(n^{2}+\lambda_{\beta m}^{\prime 2}\right)}\right] \cos n_{\pi} \eta=0 .
$$

Da diese Gleichung für jeden Wert von $\eta$ erfüllt sein soll, so muss die eckige Klammer versehwinden. Also wird

mit

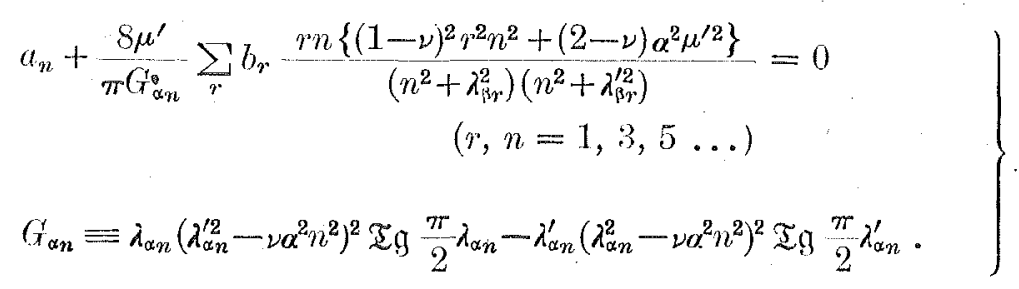

Ganz analog folgt aus den Randbedingungen 4)

mit

$$
\left.\begin{array}{c}
b_{m}+\frac{8 \mu}{\pi G_{\beta m}} \sum_{s} a_{s} \frac{m s\left\{(1-\nu)^{2} m^{2} s^{2}+(2-\nu) \beta^{2} \mu^{2}\right\}}{\left(m^{2}+\lambda_{\alpha_{s}}^{2}\right)\left(m^{2}+\lambda_{\alpha_{s}}^{2}\right)}=0 \\
(m, s=1,3,5 \ldots) \\
G_{\beta_{m}} \equiv \lambda_{\beta m}\left(\lambda_{\beta m}^{\prime 2}-\nu \beta^{2} m^{2}\right)^{2} \mathfrak{I} \mathfrak{g} \frac{\pi}{2} \lambda_{\beta m}-\lambda_{\beta m}^{\prime}\left(\lambda_{\beta m}^{2}-\nu \beta^{2} m^{2}\right)^{2} \mathfrak{I} \mathfrak{g} \frac{\pi}{2} \lambda_{\beta m}^{\prime} \cdot
\end{array}\right\}
$$

In den vorangehenden Gleichungen (12) und (13) ist in den Summationszeichen $\sum r$ bzw. $s$ an Stelle von $m$ bzw. $n$ geschrieben, um weiterhin Verwechslungen zu vermeiden.

Wenn man nun den aus (13) hervorgehenden Ausdruck für $b_{r}\left(=b_{m}\right)$ in (12) einsetzt, so erhält man die Gleichung für den einzigen Beiwert $a_{n}$, wie folgt:

$$
a_{n}+\sum_{s} K_{n s} a_{s}=0, \quad(n, s=1,3,5 \ldots)
$$

mit

$$
K_{n s}=-\frac{64 \mu \mu^{\prime} n s}{\pi^{2} G_{\alpha_{n}}} \sum_{r} \frac{r^{2}\left\{(1-\nu)^{2} r^{2} n^{2}+(2-\nu) \alpha^{2} \mu^{\prime 2}\right\}\left\{(1-\nu)^{2} r^{2} s^{2}+(2-\nu) \beta^{2} \mu^{2}\right\}}{G_{\beta r}\left(n^{2}+\lambda_{\beta r}^{2}\right)\left(n^{2}+\lambda_{\beta r}^{\prime 2}\right)\left(\gamma^{2}+\lambda_{\alpha_{s}}^{2}\right)\left(r^{2}+\lambda_{\alpha s}^{2}\right)} .
$$

Die erste Gleichung in (14) stellt ein System von unendlich vielen homogen linearen Gleichungen für die unbekannten Beiwerte $a_{n}\left(=a_{1}, a_{3}, a_{5}, \cdots\right)$ dar. Da nicht alle $a_{n}$ verschwinden können, so muss die aus den Koeffizientien bestehende Determinante verschwinden. Also erhält man

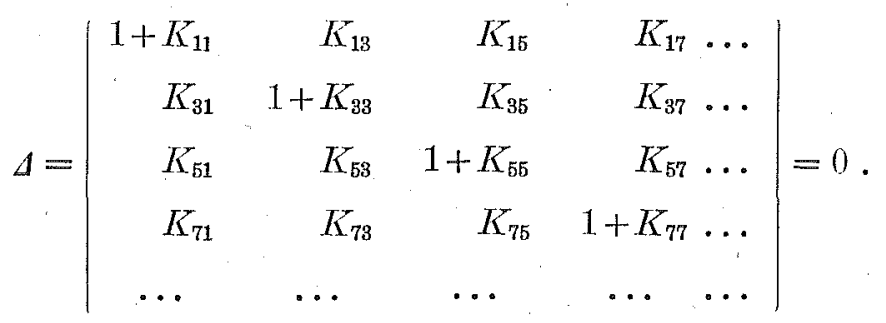


Dies ist die gesuchte Frequenzgleichung, durch deren Auflösung man die den verschiedenen Tonhöhen dieser Schwingungsart entsprechenden Eigenwerte $\mu$ (oder $\mu^{\prime}$ ) aufzufinden hat. Wenn man so die Werte von $\mu$ ausrechnet, kann man leicht die Kreisfrequenz, die Schwingungsdauer bzw. -frequenz mittels der bekannten Formeln

$$
\omega=\frac{\pi^{2} \mu}{a^{2}} \sqrt{\frac{\bar{N}}{\rho / l}} \quad T=\frac{2 \pi}{\omega}, \quad \text { bzw. } \quad f=\frac{\omega}{2 \pi}
$$

ermitteln und die zu jedem Eigenwert gehörenden Schwingungsflächen der Platte vollständig bestimmen. So muss es die Hauptaufgabe der weiteren Rechnungen sein, die den verschiedenen Schwingungen entsprechenden Werte von $\mu$ auszurechnen. Die wirklichen numerischen Rechnungen werden dann bei der Untersuchung der quadratischen Platte in der nächsten Ziff. angegeben.

6. Die Formeln und die numerischen Rechenergebnisse für die quadratische Platte. Wegen der Sonderbeziehungen $m=n=1,3,5 \cdots, \alpha=\beta=1$, $\mu^{\prime}=\mu$ für die quadratische Platte kann man in allen Formeln hierfür einfachheitshalber $\lambda_{n}$ fuir $\lambda_{\alpha_{n}}$ and $\lambda_{\beta m}$, $\lambda_{n}^{\prime}$ firr $\lambda_{\alpha_{n}}^{\prime}$ und $\lambda_{\beta m}^{\prime}$, $u_{n}$ für $\mu_{\alpha_{n}}$ und $u_{\beta m}$ schreiben. So lautet nach $(10)$

$$
\begin{gathered}
w^{\prime}=\sum_{n}(-1)^{\frac{n-1}{2}}\left\{a_{n} u_{n}(\xi) \cos n \pi \eta+b_{n} u_{n}(\eta) \cos n \pi \xi\right\} . \\
(n=1,3,5 \ldots)
\end{gathered}
$$

Der entsprechende Ausdruck für $K_{n s}(14)$ schreibt sich

$$
\left.\begin{array}{l}
K_{n s}=-\frac{64 \mu^{2} n s}{\pi^{2} G_{n}} \sum_{r} \frac{r^{2}\left\{(1-\nu)^{2} r^{2} n^{2}+(2-\nu) \mu^{2}\right\}\left\{(1-\nu)^{2} r^{2} s^{2}+(2-\nu) \mu^{2}\right\}}{G_{r}\left\{\left(r^{2}-n^{2}\right)^{2}-\mu^{2}\right\}\left\{\left(r^{2}+s^{2}\right)^{2}-\mu^{2}\right\}} \\
\cdot \\
G_{n} \equiv \lambda_{n}\left(\lambda_{n}^{\prime 2}-\nu n^{2}\right)^{2} \mathfrak{I} \mathfrak{g} \frac{\pi}{2} \lambda_{n}-\lambda_{n}^{\prime}\left(\lambda_{n}^{2}-\nu n^{2}\right)^{2} \mathfrak{I} \mathfrak{g} \frac{\pi}{2} \lambda_{n}^{\prime} \cdot
\end{array}\right\}
$$

Der Ausdruck für $G_{r}$. ist derselbe wie der für $G_{n}$ mit $r$ an Stelle von $n$.

Um nun die Eigenschaft der Determinantengleichung (15) mit diesen Wert von $K_{n s}$ zu untersuchen, setzen wir vorteilhafterweise

mit

$$
K_{n s}=-\sum_{r} \phi_{r n} \phi_{r s}
$$

$$
\left.\begin{array}{l}
\mathscr{Q}_{r n}=\frac{8 \mu \eta^{2} n}{\pi\left(x_{n}\right.} \frac{(1-\nu)^{2} r^{2} n^{2}+(2-\nu) \mu^{2}}{\left(r^{2}+n^{2}\right)^{2}-\mu^{2}}, \\
\mathscr{t}_{r s}=\frac{8 \mu \eta \cdot s}{\pi G_{r}} \frac{(1-\nu)^{2} r^{2} s^{2}+(2-\nu) \mu^{2}}{\left(r^{2}+s^{2}\right)^{2}-\mu^{2}} .
\end{array}\right\}
$$

Damit drückt sich jetzt die Gleichung (15), wie folgt, aus : 


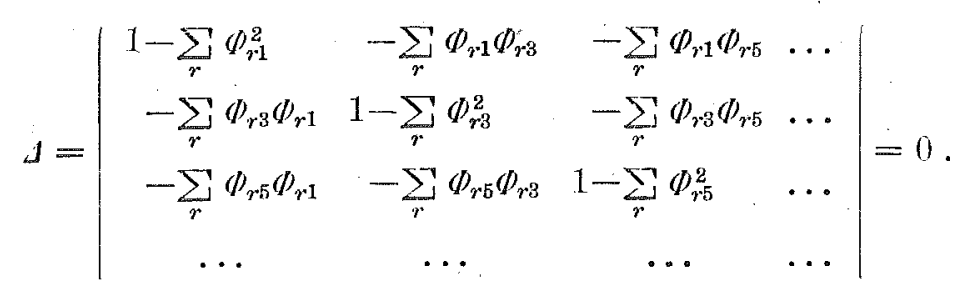

Mit Hilfe der Multiplikationsformel der zwei Determinanten und wegen der Symmetrierelation $\Phi_{i j}=\Phi_{j i}$ können wir die obige Gleichung in zwei Faktoren

$$
\left|\begin{array}{rrrr}
1+\Phi_{11} & \Phi_{13} & \Phi_{15} & \ldots \\
\Phi_{31} & 1+\Phi_{33} & \Phi_{35} & \ldots \\
\Phi_{51} & \phi_{53} & 1+\Phi_{55} & \ldots \\
\ldots & \ldots & \ldots & \ldots
\end{array}\right| \times\left|\begin{array}{rrrr}
1-\Phi_{11} & -\Phi_{13} & -\Phi_{15} & \ldots \\
-\Phi_{31} & 1-\Phi_{33} & -\Phi_{35} & \ldots \\
-\Phi_{51} & -\Phi_{53} & 1-\Phi_{55} & \ldots \\
\ldots & \ldots & \ldots & \ldots
\end{array}\right|=0
$$

zerlegen, aus welcher die zwei verschiedenen Determinantengleichungen

$$
\begin{aligned}
& J^{(1)}=\left|\begin{array}{rrrr}
1+\Phi_{11} & \Phi_{13} & \phi_{15} & \cdots \\
\Phi_{31} & 1+\Phi_{32} & \Phi_{35} & \ldots \\
\Phi_{51} & \Phi_{53} & 1+\Phi_{55} & \ldots \\
\ldots & \ldots & \ldots & \ldots
\end{array}\right|=0 \\
& J^{(2)}=\left|\begin{array}{cccc}
1-\Phi_{11} & -\Phi_{13} & -\Phi_{15} & \ldots \\
-\Phi_{31} & 1-\Phi_{33} & -\Phi_{35} & \ldots \\
-\Phi_{51} & -\Phi_{53} & 1-\Phi_{55} & \ldots \\
\ldots & \ldots & \ldots & \ldots
\end{array}\right|=0
\end{aligned}
$$

folgen. Da verschiedene Reihen von Wurzeln $\mu$ aus (19) und (20) hervorgehen, so sagt die obige Tatsache aus, dass es bei dieser Schwingungsart zwei verschiedene Schwingungsfamilie gibt, deren nähere Untersuchungen jetzt folgen.

1) Die erste Schwingungsfamilie. Es handelt sich nun um die erste Familie der ersten Schwingungsart, in der die Platte symmetrisch in bezug nicht nur auf ihre beiden Mittellinien, sondern auch auf ihre beiden Diagonalen schwingt. Die Funktion $w^{\prime}$ für eine derartige Schwingungsfäche hat die Symmetrieeigenschaft

$$
w^{\prime}(x, y)=w^{\prime}(y, x)
$$

oder aus (16)

$$
w^{\prime}(x, y)-w^{\prime}(y, x)=\sum_{n}(-1)^{\frac{n-1}{2}}\left(a_{n}-b_{n}\right)\left\{u_{n}(\xi) \cos n \pi \eta-u_{n}(\eta) \cos n \pi \xi\right\}=0 .
$$


Diese Forderung wird erfullt, wenn $a_{n}=b_{n}$ ist. Somit vereinfacht sich der A usdruck für: $w^{\prime}(16)$, wie folgt :

$$
\begin{gathered}
w^{\prime}=a_{1} \bar{w}^{\prime}, \\
\bar{w}^{\prime}=\sum_{n}(-1)^{\frac{n-1}{2}} \alpha_{n}\left\{u_{n}(\xi) \cos n \pi \eta+u_{n}(\eta) \cos n \pi \xi\right\}, \\
\quad \alpha_{n}=\frac{a_{n}}{a_{1}}, \quad\left(\alpha_{1}=1\right), \quad(n=1,3,5 \ldots) .
\end{gathered}
$$

Wegen $b_{n}\left(=b_{m}\right)=a_{n}$ folgt aus (12) oder (13)

$$
a_{n}+\frac{8 \mu}{\pi G_{n}} \sum_{s} a_{s} \frac{n s\left\{(1-\nu)^{2} n^{2} s^{2}+(2-\nu) \mu^{2}\right\}}{\left(n^{2}+s^{2}\right)^{2}-\mu^{2}}=0 .
$$

Damit druickt sich $K_{n s}$ in der ersten Gleichung (14) aus, wie folgt :

$$
K_{n s}=\frac{8 \mu n s}{\pi G_{n}} \frac{(1-\nu)^{2} n^{2} s^{2}+(2-\nu) \mu^{2}}{\left(n^{2}+s^{2}\right)^{2}-\mu^{2}} \quad(n, s=1,3,5 \ldots) .
$$

Die Vergleichung dieses Ausdrucks für $K_{n s}$ mit dem für $\phi_{r n}$ oder $\phi_{r s}$ in (18) zeigt, dass die Frequenzgleichung für den betrachteten Schwingungsfall identisch mit der durch (19) dargestellten Gleichung wird. So bestätigt man, dass die zur ersten Schwingungsfamilie gehörenden Eigenwerte $\mu$ auch in der durch den allgemeinen Ausdruck für $K_{n s}$ (17) gebildeten Frequenzgleichung enthalten sind.

Wir gehen jetzt dazu über, die Werte von $\mu$ aufzufinden. Aus (15) und (22) erkennt man, dass es sehr schwer ist, die genauen Werte von $\mu$ unmittelbar: aus ihr selbst, nämlich aus einer Determinantengleichung mit unendlich vielen Elementen auszurechnen. Also nehmen wir zuerst einmal

$$
\Delta_{1}=1+K_{11}=0
$$

als die Frequenzgleichung an, deren Wurzeln die ersten Näherungswerte von $\mu$ sein sollen. Durch Einführung des Ausdrucks (22) für $K_{n s}$ mit $n=s=1$ ergibt sich

$$
\Delta_{1}=1+\frac{8 \mu}{\pi} \frac{(1-\nu)^{2}+(2-\nu) \mu^{2}}{\left(4-\mu^{2}\right)\left\{(1-\nu-\mu)^{2} \sqrt{1+\mu} \mathfrak{I} \mathfrak{V} \frac{\pi}{2} \sqrt{1+\mu}-(1-\nu+\mu)^{2} \sqrt{1-\mu} \mathfrak{I} \mathfrak{g} \frac{\pi}{2} \sqrt{1-\mu}\right\}}=0
$$

oder

$$
(1-\nu+\mu)^{2} \sqrt{1-\mu} \mathfrak{I} \mathfrak{g} \frac{\pi}{2} \sqrt{1-\mu}=\varphi(\mu)
$$

mit

$$
\phi(\mu)=\frac{8 \mu}{\pi} \frac{(1-\nu)^{2}+(2-\nu) \mu^{2}}{4-\mu^{2}}+(1-\nu-\mu)^{2} \sqrt{1+\mu} \mathfrak{I} \mathfrak{g} \frac{\pi}{2} \sqrt{1+\mu} .
$$

Wir haben also die Schnittpunkte der heiden Kurven 


$$
\left.\begin{array}{l}
\zeta_{1}=\mathfrak{I g} \frac{\pi}{2} \sqrt{1-\mu}, \\
\zeta_{2}=\frac{\varphi(\mu)}{(1-\nu+\mu)^{2} \sqrt{1-\mu}}
\end{array}\right\} \quad \text { für } \mu<1
$$

zu finden. Die wirkliche Durchrechnung für die quadratische Platte mit $\nu=0,3$ liefert die durch Abb. 2 dargestellten Kurven, aus denen man die folgenden Näherungswurzeln ermittelt :

$$
\begin{aligned}
& \text { 1. Wurzel } \sqrt{1-\mu}=0,52, \quad \mu=0,730, \\
& \text { 2. Wurzel } \sqrt{\mu-1}=1,89, \quad \mu=4,572, \\
& \text { 3. Wurzel } \sqrt{\mu-1}=3,58, \quad \mu=13,816 .
\end{aligned}
$$

Diese Ergebnisse gewähren uns bei weiteren Rechnungen einen guten Anhalt.

Nun handelt, es sich um die genanere Ausrechnung der Werte von $\mu$. Bezeichnen wir mit $\boldsymbol{\Delta}_{1}$, wie oben das erste Element der Determinante (15), d.h. $1+K_{11}$, und mit $\lrcorner_{2}, d_{3}, \Delta_{4}$ die aus den ersten, $2,3,4$ Zeilen und Spalten bestehende Determinante. Zur Ermittlung von $\mu$ rechnet man zunächst für jede dieser Determinanten der

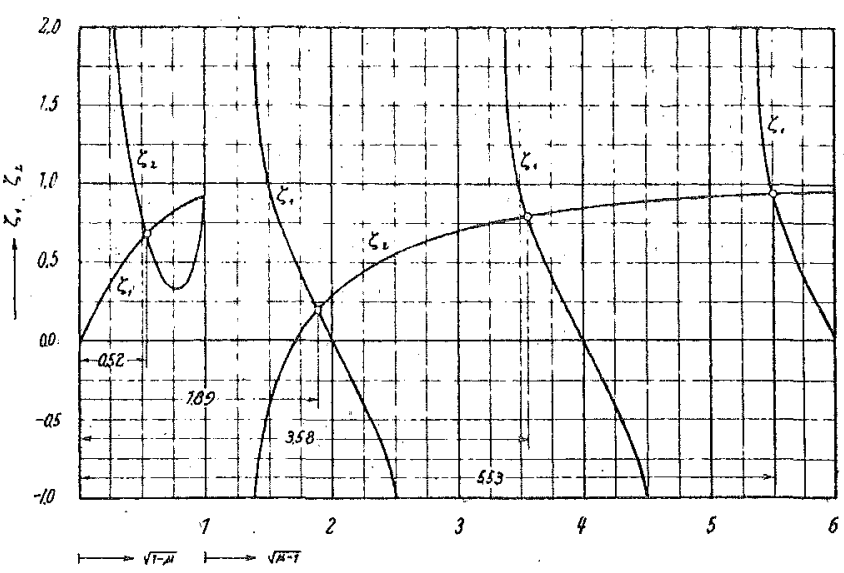

Abb. 2. Kurven zur Ermittlung der Werte von $\sqrt{1-\mu}$ und $\sqrt{\mu-1}$ für die quadratische Platte mit $v=0,3$. Reihe nach die Werte aus, welche den versuchsweise gewählten Werten $\mu=0,750 ; 0,740 ; 0,716$ und 0,700 zum Beispiel für die erste Wurzel entsprechen.1). Die Ergebnisse sind in folgender 'Tabelle zusammengestellt.

1) Vgl. S. Iguchi, Ing.-Arch. 7 (1936) S. 207 ; 8,(1937) S. 11. 
Tabelle 1. Die Werte der den verschiedenen Grössen von $\mu$ entsprechenden Determinanten $(\nu=0,3)$.

\begin{tabular}{c|c|c|c|c}
\hline$\mu$ & \multicolumn{1}{|c|}{$\Delta_{\mathbf{1}}$} & \multicolumn{1}{c|}{$\Delta_{\mathbf{2}}$} & \multicolumn{1}{c}{$\Delta_{\mathbf{3}}$} & \multicolumn{1}{c}{$\Delta_{\mathbf{A}}$} \\
\hline 0,750 & $-0,170988$ & $-0,166049$ & $-0,161688$ & $-0,158508$ \\
0,740 & $-0,106968$ & $-0,104873$ & $-0,102209$ & $-0,100218$ \\
0,716 & 0,025997 & 0,022201 & 0,021341 & 0,020863 \\
0,700 & 0,101356 & 0,094224 & 0,091369 & 0,089494 \\
\hline Wurzeln & 0,721086 & 0,720555 & 0,720503 & 0,720491 \\
\hline
\end{tabular}

Aus dieser Tabelle sieht man leicht, dass die erste Wurzel jeder Determinantengleichung zwischen 0,740 und 0,716 liegt. Die in der untersten Reihe der Tabelle angeschriebenen Ziffern sind die Werte der Wurzeln, welche mittels der Lagrangeschen Interpolationsformel ausgerechnet sind. Ferner zeigt die Tabelle, dass die Konvergenz der Zahlenfolge der den Determinantengleichungen $\Delta_{1}=0, \quad \Delta_{2}=0, \quad \Delta_{3}=0, \quad \Delta_{4}=0$ entsprechenden Wurzeln recht gut ist, wie $A$ bb. 3 zeigt; aus $\Delta_{5}=0$ würde mit Hilfe der Newtonschen Interpolationsformel der gleiche Wert $\mu=0,720491$ folgen, genau wie $\mu$ aus $\lrcorner_{4}=0$. Somit kann man $\mu=0,72049$ als den richtigen Wert der ersten Wurzel annehmen. Dieser Wert von $\mu$ ist der allerniedrigste Eigenwert aller Schwin-

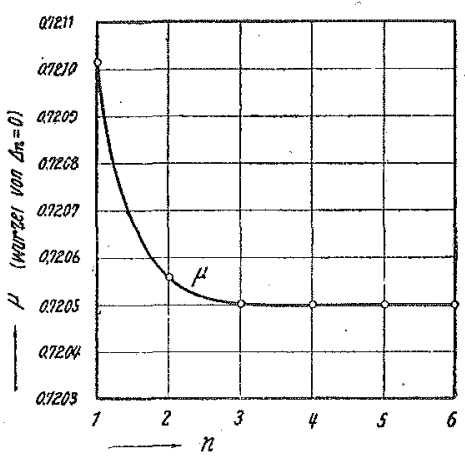

Abb. 3. Dar'stellung der Güte der Konvergenz der Zahlenfolge dex Wurzeln $\mu$ ans $\Delta_{n}=0$. gungsarten der betrachteten quadratischen Platte, also gehört er dem Grundton an, in dem die Platte ohne Knotenlinie schwingt, wie Abb. 4-(I) oder 5-(I) zeigt.

Auf Grund der voran erwähnten Tatsache überzeugt man sich nun, dass man einen hinreichend genauen Wert von $\mu$ erhält, wenn man nur die ersten drei oder vier Zeilen und Spalten der Determinante nimmt. Das gilt auch für die anderen Schwingungsarten, obwohl die Konvergenz gewöhnlich mit wachsendem $\mu$ etwas schlechter wird. In den nachfolgenden numerischen Rechnungen benutze ich immer die Determinante mit den ersten vier Zeilen und Spalten, d.h. die Frequenzgleichung $\Delta_{4}=0$. Die so ausgerechneten höheren Eigenwerte $\mu$ sind in Tabelle 2 zusammengestellt. 
'Tabelle 2. Die Werte von $\mu$ und die der verschiedenen Beiwerte für die erste Familie der ersten Schwingungsart der quadratischen Platte mit $\nu=0,3$.

\begin{tabular}{|c|c|c|c|c|c|c|c|}
\hline$\mu$. & $n$ & $\alpha_{n}$ & $\lambda_{n}$ & $x^{\prime \prime}{ }_{n}-v n^{2}$ & $x_{n}$ & $\lambda_{n}^{2}-v n^{2}$ & $\begin{array}{l}\text { Abb. } 4 . \\
\text { Knotenlinien. }\end{array}$ \\
\hline \multirow{4}{*}{ 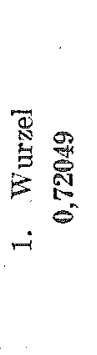 } & 1 & 1,000000 & 1,311674 & $-0,02049$ & 0,528687 & 1,42049 & \\
\hline & 3 & 0,006940 & 3,117770 & 5,57951 & 2,877414 & 7,02049 & \\
\hline & 5 & 0,000982 & 5,071538 & 16,77951 & 4,927424 & 18,22049 & \\
\hline & 7 & 0,000271 & 7,051276 & 33,57951 & $6,94834.5$ & 35,02049 & (I) \\
\hline \multirow{4}{*}{ 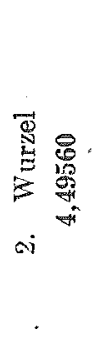 } & 1 & 1,000000 & 2,344269 & $-3,79560$ & $1,869652{ }^{*}$ & 5,19560 & \\
\hline & 3 & 0,080207 & 3,673636 & 1,80440 & 2,122357 & 10,79560 & \\
\hline & 5 & 0,004760 & 5430986 & 13,00440 & 4,528178 & 21,99560 & \\
\hline & 7 & 0,001023 & 7,314069 & 29,80440 & 6,671161 & 38,79560 & (II) \\
\hline \multirow{4}{*}{ 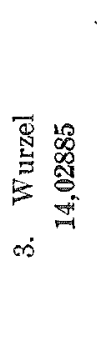 } & 1 & 1,000000 & 3,876706 & $-13,32885$ & 3,609550 i & 14,72885 & \\
\hline & 3 & 0,223594 & 6,798838 & $-7,72885$ & $2,242510 i^{*}$ & 20,32885 & \\
\hline & 5 & 0,062859 & 6,217307 & 3,47115 & 3,312272 & 31,52886 & \\
\hline & 7 & 0,007986 & 7,939070 & 20,27115 & 5,913643 & 48,32885 & (III) \\
\hline
\end{tabular}

Weiter wollen wir uns mit der Untersuchung der Schwingungsgestalten dieser Familie beschäftigen. Zu diesem Zwecke genügt es, nur die Funktion $\bar{w}^{\prime}$ (21) anstatt von $y^{\prime}$ (16) zu betrachten, denn die wirliche Auslenkung des Punktes $(x, y)$ zu irgendeiner Zeit ist proportional zu $\bar{x}^{\prime}$. So hat man es nun nötig, die Werte der unbekannten Beiwerte $\alpha_{n}$ auszurechnen. Dividiert man $z u$ diesem Zwecke die erste Gleichung in (14) durch $a_{1}$, so erhält man

$$
\alpha_{n}+\sum_{s} K_{n s} \alpha_{s}=0, \quad\left(a_{1}=1\right), \quad(n, s=1,3,5,7)
$$

oder mit den vorteilhafteren Bezeichnurig, $c_{n s}=K_{n s}$ für $n \neq s ; c_{n n}=1+K_{n n}$ für $n=s$, schreibt sich 


$$
\left.\begin{array}{l}
c_{11}+c_{13} \alpha_{3}+c_{15} \alpha_{5}+c_{17} \alpha_{7}=0, \\
c_{31}+c_{33} \alpha_{3}+c_{25} \alpha_{5}+c_{37} \alpha_{7}=0, \\
c_{51}+c_{53} \alpha_{3}+c_{55} \alpha_{5}+c_{57} \alpha_{7}=0, \\
c_{71}+c_{73} \alpha_{3}+c_{75} \alpha_{5}+c_{77} \alpha_{7}=0 .
\end{array}\right\}
$$

Dies lautet zum Beispiel für die erste Wurzel $\mu=0,72049$

$$
\begin{aligned}
0,003090-0,403587 \alpha_{3}-0,245779 \alpha_{5}-0,176261 \alpha_{7} & =0 \\
-0,006545+0,953707 \alpha_{5}-0,059165 \alpha_{5}-0,055555 \alpha_{7} & =0 \\
-0,000858-0,012729 \alpha_{3}+0,972895 \alpha_{5}-0,033904 \alpha_{7} & =0 \\
-0,005224-0,004354 \alpha_{3}-0,012351 \alpha_{5}+0,980691 \alpha_{7} & =0
\end{aligned}
$$

Diese vier Gleichungen für die drei Unbekannten $\alpha_{5}, \alpha_{5}, \alpha_{7}$ liefern vier verschiedene Systeme der drei Gleichungen, aus deren Lösungen vier Wurzeln für jede Unbekannte hervorgehen. Abor sie müsson untereinander gleich sein. wenn die Bedingung (Frequenzgleichung), dass die aus allen Koeffizienten bestehende Determinante $J_{4}$ gleich Null wird, genau erfüllt würde. Aber es ist fast unmöglich, die Wurzeln $\mu$, mit denen die Determinante genau gleich. Null wird, auszufinden. So zum Beispiel stellt die wirkliche Rechnung

$$
s_{4}=\left\{\begin{aligned}
0,000001 & \text { für die erste Wurzel } \mu=0,72049, \\
-0,000037 & \text { für die zweite Wurzel } \mu=4,49560, \\
0,000075 & \text { für die drite Wurzel } \mu=14,02985
\end{aligned}\right.
$$

dar. Mithin muss ein Fehler auf der rechten Seite jeder Gleichung (24) oder • (a) bis (d) himzugefügt werden, und die wahrscheinlichsten Werte von $\alpha_{3}, \alpha_{5}, \alpha_{7}$ sind aus den Normalgleichungen

$$
\left.\begin{array}{l}
{\left[c_{n 3} c_{n 1}\right]+\left[c_{n 3} c_{n 3}\right] \alpha_{3}+\left[c_{n 3} c_{n 5}\right] \alpha_{5}+\left[c_{n 3} c_{n 7}\right] \alpha_{7}=0,} \\
{\left[c_{n 5} c_{n 1}\right]+\left[c_{n 5} c_{n 3}\right] \alpha_{3}+\left[c_{n 5} c_{n 5}\right] \alpha_{5}+\left[c_{n 5} c_{n 7}\right] \alpha_{7}=0,} \\
{\left[c_{n 7} c_{n 1}\right]+\left[c_{n 7} c_{n 3}\right] \alpha_{3}+\left[c_{n 7} c_{n 5}\right] \alpha_{5}+\left[c_{n 7} c_{n 7}\right] \alpha_{7}=0}
\end{array}\right\}
$$

$z u$ finden. So lauten die den Gleichungen $(a)$ bis $(d)$ entsprechenden Normalgleichungen mit verhundertfachten Koeffizienten, wie folgt:

$$
\begin{aligned}
& -0,7477045+107,2580137 \alpha_{3}+3,0424641 \alpha_{5}+1,4306278 \alpha_{7}=0, \\
& -0,1204199+3,0424641 \alpha_{3}+101,0432495 \alpha_{5}+0,1510617 \alpha_{7}=0, \\
& -0,0371624+1,4306278 \alpha_{3}+0,1510617 \alpha_{5}+99,7058617 \alpha_{7}=0,
\end{aligned}
$$

aus deren Lösung man die gesuchten Wurzeln

$$
x_{3}=0,006940, \quad \alpha_{5}=0,000982, \quad a_{7}=0,000271
$$

erhält. In analoger Weise kann man die Werte von $\boldsymbol{\alpha}_{3}, \boldsymbol{\alpha}_{5}, \boldsymbol{\alpha}_{7}$ für die höheren Eigenwerte $\mu$ ausrechnen. Die Ergebnisse sind in Tabelle 2 zusammengestellt. 


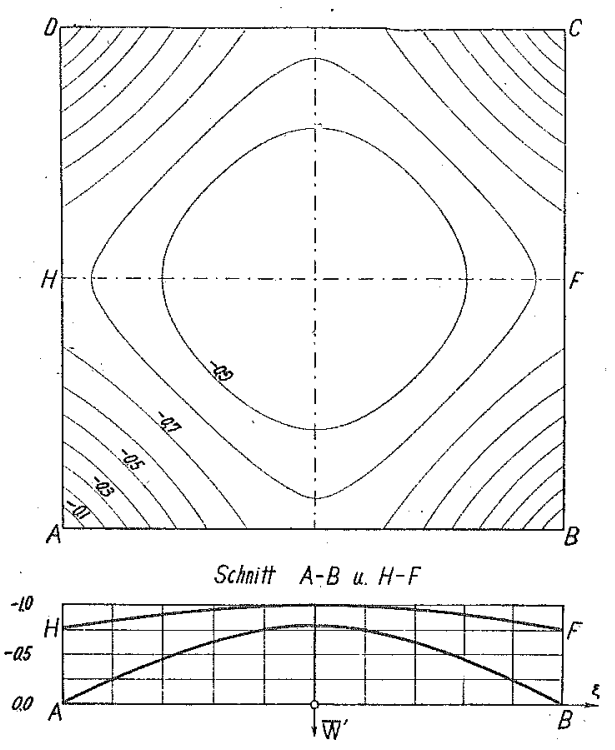

(I). $\mu_{1}=0,72049$.

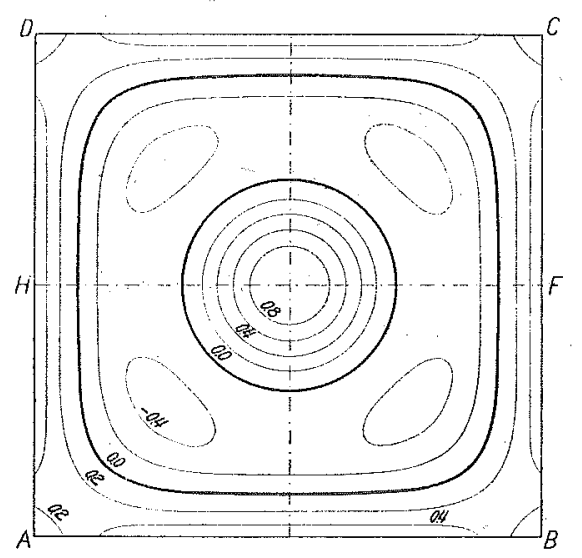

Schnitt $A-B$ u. $H-F$

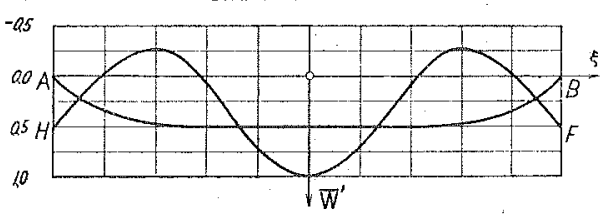

(III). $\mu_{3}=14,02885$.

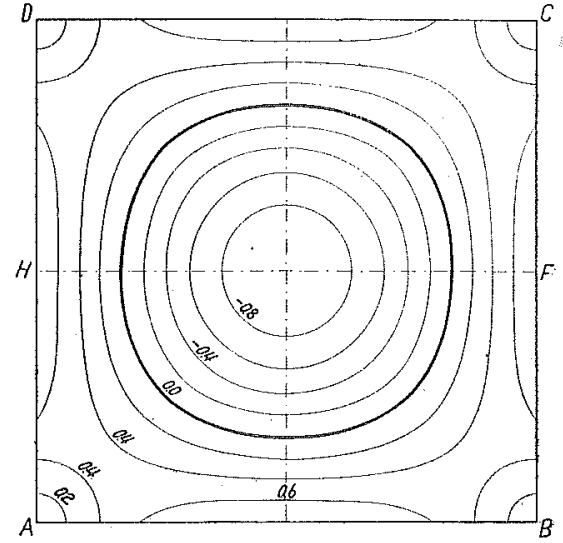

Schnitt $A-B$ u. H-F

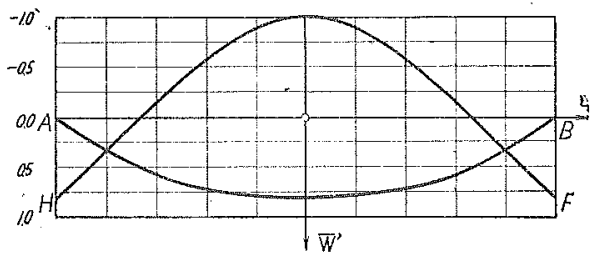

(II). $\mu_{2}=4,49560$.

Abb. 5. Schichtlinien der $\bar{w}^{\prime}-$ Fläche für die erste Familie der ersten Schwingungsart.

Nachdem die Werte von $\mu$ und $a_{n}$ ermittelt sind, liegt es nahe, die Auslenkungen $\bar{w}^{\prime}(21)$ mit $u_{n}(\xi)$ in (8) und $u_{n}(\eta)$ in (9) auszurechnen und daraus die Knotenlinien $\bar{v}^{\prime}=0$ zu bestimmen. Die so gebildeten Knotenfiguren für die ersten drei Wurzeln werden schematisch in Abb. 4 und die entsprechenden Schichtlinien der $\bar{w}^{\prime}-$ Fläche (mit dem gxössten Wert von $\bar{w}^{\prime}$ àls Einheit) durch Abb. 5-(I) bis 5(III) dargestellt. Aus Abb. 5-(I) erkennt man, dass es in der der niedrigsten Wurzel $\mu=0,72049$ (also dem Grundton) angehörenden Schichtlinien keine Knotenlinie gibt.

2) Die zweite Schwingungsfamilie. Wir wollen uns zweitens mit der zweiten Familie der ersten Schwingungsart beschäftigen, in welcher die Platte symmetrisch in bezug auf die beiden Mittellinien, antisymmetrisch dagegen in bezug auf die beiden Diagonalen des Quadrats schwingt. Selbstverständlich hat 
eine solche Schwingungsfläche mindestens zwei Diagonalen als Knotenlinien, und es gilt offenbar die Antisymmetriebedingung

$$
w^{\prime}(x, y)=-w^{\prime}(y, x)
$$

die durch die Sonderrelation

$$
b_{n}\left(=b_{m}\right)=-a_{n}
$$

befriedigt wird. Somit ergibt sich aus (16)

$$
\left.\begin{array}{c}
w^{\prime}=a_{1} \bar{w}^{\prime} \\
\bar{w}^{\prime}=\sum_{n}(-1)^{\frac{n-1}{2}} a_{n}\left\{u_{n}(\xi) \cos n \pi \eta-u_{n}(\eta) \cos n \pi \xi\right\}, \\
\alpha_{n}=\frac{a_{n}}{a_{1}}, \quad\left(\alpha_{1}=1\right), \quad(n=1,3,5 \ldots) .
\end{array}\right\}
$$

Dieser Ausdruck für $\bar{w}^{\prime}$ unterscheidet sich von dem für $\bar{w}^{\prime}$ (21) nur in Vorzeichen von $u_{n}(\eta)$ und der Ausdruck für $K_{n s}$ ist auch derselbe wie (22) mit entgegengesetztem Vorzeichen. Ferner sieht man leicht, dass die durch diese Änderung hergestellte Frequenzgleichung identisch mit der Gleichung $j^{(2)}=0$ (20) wird. Somit bestätigt man jetzt, dass die zu der ersten sowie der zweiton Familie dieser Schwingungsart gehörenden Eigenwerte $\mu$ in der durch den

\begin{tabular}{|c|c|c|c|c|c|c|c|}
\hline$\mu$ & $n$ & $\alpha_{n}$ & $\lambda_{n}$ & $x^{\prime 2}{ }_{n-v n^{2}}$ & $Y_{n}^{\prime}$ & $\lambda^{2} n-v n^{2}$ & $\begin{array}{c}\text { Abb. } 6 . \\
\text { Knotenlinien. }\end{array}$ \\
\hline \multirow{4}{*}{ 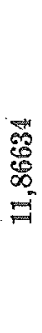 } & 1 & 1,000000 & 3,586967 & $-11,16634$ & $3,296413 i$ & 12,56634 & \\
\hline & 3 & 0,979573 & 4,567969 & $-5,56634$ & $1,693027 i$ & 18,16634 & \\
\hline & 5 & $-0,071498$ & 6,071765 & 5,63366 & 3,624039 & 29,36634 & \\
\hline & 7 & $-0,010726$ & 7,801694 & 22,43366 & 6,093738 & 46,16634 & \\
\hline
\end{tabular}
allgemeinen Ausdruck für $K_{n s}$ (17) gebildeten Frequenzgleichung enthalten sind.

Tabelle 3. Die Werte der verschiedenen Beiwerte für die zweite Familie der ersten Schwingungsart der quadratischen

Platte mit $\nu=0.8$.

Die numerischen Rechenverfahren für $\mu, \alpha_{n}$ u.s.w. für diese Familie sind auch ganz analog wie für die früheren Fälle. So sind nur die Rechenergebnisse für den ersten Eigenwert $\mu=11,86634$ in Tabelle 3 zusammengestellt Die zu diesem Eigenwert gehörende Knotenfigur wird schematiseh durch Abb. 6 gezeigt. Abb. 7 stellt die entsprechenden Schichtlinien der $\bar{w}^{\prime}$-Fläche dar. 

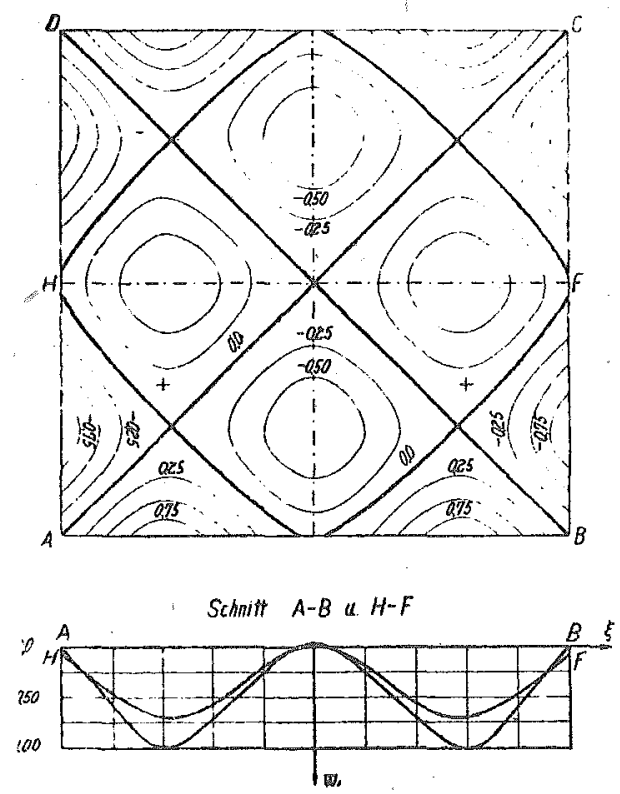

Abb. 7. Schichtlinien der $\bar{u}^{\prime}-$ Fläche für die zweite Familie der ersten Schwingungsart mit $\mu=11,86634$.

\section{Die zweite Schwingungsart.}

7. Die allgemeinen Formeln für die rechteckige Platte. Es handelt sich jetzt um die zwoite Schwingungsart, in der die Schwingungsläche antisymmetrisch in bezug auf die beiden Mittellinien $\xi=0$ und $\eta=0$ des Rechtecks ist. Eine derartige Schwingungsfläche hat mindestens zwei Knotenlinien $\xi=0$ und $\eta=0$ und wird wegen $m=n=2,4,6 \cdots, a_{n}=0, b_{m}=0$ durch

mit

$$
\begin{gathered}
w^{\prime}=\sum_{n}(-1)^{\frac{n}{2}} a_{n}^{\prime} v_{\alpha n}(\xi) \sin n \pi \eta+\sum_{m}(-1)^{\frac{m}{2}} b_{m}^{\prime} v_{\beta m}(\eta) \sin m_{\pi} \xi \\
(m, n=2,4,6 \ldots)
\end{gathered}
$$

$$
\begin{aligned}
& v_{\alpha n}(\xi)=\left(\lambda_{\alpha, n}^{\prime 2}-\nu \alpha^{2} n^{2}\right) \frac{\operatorname{Sin} \pi \lambda_{\alpha n} \xi}{\operatorname{Sin} \frac{\pi}{2} \lambda_{\alpha n}}-\left(\lambda_{\alpha n}^{2}-\nu \alpha^{2} n^{2}\right) \frac{\operatorname{Sin} \pi \lambda_{\alpha n}^{\prime} \xi}{\operatorname{Sin} \frac{\pi}{2} \lambda_{\alpha n}^{\prime}}, \\
& \left.v_{\beta m}(\eta)=\left(\lambda_{\beta m}^{\prime 2}-\nu \beta^{2} m^{2}\right) \frac{\operatorname{Sin} \pi \cdot \lambda_{\beta m} \eta}{\operatorname{Sin} \frac{\pi}{2} \lambda_{\beta m}}-\left(\lambda_{\beta m}^{2}-\nu \beta^{2} m^{2}\right) \frac{\operatorname{Sin} \pi \lambda_{\beta m}^{\prime} \eta}{\operatorname{Sin} \frac{\pi}{2} \lambda_{\beta m}^{\prime}}\right)
\end{aligned}
$$

ausgedriickt. Da diese Gleichung von vornherein den Randbedingungen 1) und 2. genügt, so müssen die beiden unbestimmten Beiwerte $a_{n}^{\prime}$ und $b_{n}^{\prime}$ zweckmässig so verknüpft werden, dass $w^{\prime}$ (27) den übrigen Randbedingungen 3) bzw. 4), nämlich. 


$$
\begin{aligned}
\sum_{n}(-1)^{\frac{n}{2}} a_{n}^{\prime}\left\{\frac{a^{3}}{\pi^{3}} v_{\alpha_{n}}^{\prime \prime \prime}\left( \pm \frac{1}{2}\right)\right. & \left.-\alpha^{2} n^{2}(2-\nu) \frac{\alpha}{\pi} v_{\alpha_{n}}^{\prime}\left( \pm \frac{1}{2}\right)\right\} \sin n \pi^{\prime \eta} \\
& -\sum_{m} b_{m}^{\prime}\left\{m^{3} v_{\beta m}(\eta)-\alpha^{2} m(2-\nu) \frac{b^{2}}{\pi^{2}} v_{\beta m}^{\prime \prime}(\eta)\right\}=0
\end{aligned}
$$

baw.

$$
\begin{aligned}
\sum_{m}(-1)^{\frac{m}{2}} b_{m}^{\prime}\left\{\frac{b^{3}}{\pi^{3}} v_{\beta m}^{\prime \prime \prime}\left( \pm \frac{1}{2}\right)-\beta^{2} m^{2}(2-\nu) \frac{b}{\pi} v_{\beta m}^{\prime}\left( \pm \frac{1}{2}\right)\right\} \sin m \pi \xi \\
-\sum_{n} a_{n}^{\prime}\left\{n^{3} v_{\alpha_{n}}(\xi)-\beta^{2} n(2-\nu) \frac{a^{2}}{\pi^{2}} v_{\alpha_{n}}^{\prime \prime}(\xi)\right\}=0
\end{aligned}
$$

genügt. In (a) lautet

$$
\begin{aligned}
& \frac{a^{3}}{\pi^{3}} v_{\alpha n}^{\prime \prime \prime}\left( \pm \frac{1}{2}\right)-\alpha^{2} n^{2}(2-\nu) \frac{a}{\pi} v_{\alpha n}^{\prime}\left( \pm \frac{1}{2}\right)=-H_{\alpha_{n}}, \quad \text { (abkürzungsweise) } \\
& =-\lambda_{\alpha n}\left(\lambda_{\alpha n}^{\prime 2}-\nu \alpha^{2} n^{2}\right)^{2} \circlearrowleft \operatorname{tg} \frac{\pi}{2} \lambda_{\alpha_{n}}+\lambda_{\alpha n}^{\prime}\left(\lambda_{\alpha n}^{2}-\nu \alpha^{2} n^{2}\right)^{2}\left(\operatorname{tg} \frac{\pi}{2} \lambda_{\alpha_{n}}^{\prime},\right. \\
& m^{3} v_{\beta m}(\eta)-\alpha^{2} m(2-\nu) \frac{b^{2}}{\pi^{2}} v_{\beta m}^{\prime \prime}(\eta)=V_{\beta m}(\eta), \quad \text { (abkürzungsweise) } \\
& =m^{3}\left(\frac{\lambda_{\beta m}^{\prime 2} \operatorname{Sin} \pi \lambda_{\beta m} \eta}{\operatorname{Sin} \frac{\pi}{2} \lambda_{\beta m}}-\frac{\lambda_{\beta m}^{2} \operatorname{Sin} \pi \lambda_{B m}^{\prime} \eta}{\operatorname{Sin} \frac{\pi}{2} \lambda_{\beta m}^{\prime}}\right) \\
& -\left\{\nu \beta^{2} m^{5}+\alpha^{2} m(2-\nu) \lambda_{\beta m}^{2} \lambda_{\beta m}^{\prime 2}\right\}\left(\frac{\operatorname{Sin} \pi \lambda_{\beta m} \eta}{\operatorname{Sin} \frac{\pi}{2} \lambda_{\beta m}}-\frac{\operatorname{Sin} \pi \lambda_{\beta m}^{\prime} \eta}{\operatorname{Sin} \frac{\pi}{2} \lambda_{\beta m}^{\prime}}\right) \\
& +\nu m^{3}(2-\nu)\left(\frac{\lambda_{\beta m}^{2} \operatorname{Sin} \pi \lambda_{\beta m} \eta}{\operatorname{Sin} \frac{\pi}{2} \lambda_{\beta m}}-\frac{\lambda_{\beta m}^{\prime 2} \operatorname{Sin} \pi \lambda_{\beta m}^{\prime} \eta}{\operatorname{Sin} \frac{\pi}{2} \lambda_{\beta m}^{\prime}}\right) .
\end{aligned}
$$

Mit Hilfe der Formeln

$$
\begin{aligned}
& \left.\frac{\lambda_{\beta m}^{\prime 2} \operatorname{Sin} \pi \lambda_{\beta m} \eta}{\operatorname{Sin} \frac{\pi}{2} \lambda_{\beta m}}-\frac{\lambda_{B m}^{2} \operatorname{Sin} \pi \lambda_{\beta m}^{\prime} \eta}{\operatorname{Sin} \frac{\pi}{2} \lambda_{\beta m}^{\prime}}=\frac{8 \mu^{\prime}}{\pi} \sum_{n}(-1)^{\frac{n}{2}} \frac{n^{3}+2 \beta^{2} m^{2} n}{\left(n^{2}+\lambda_{i m}^{2}\right)\left(n^{2}+\lambda_{\beta m}^{\prime 2}\right)} \operatorname{Sin} n \pi \eta,\right) \\
& \frac{\operatorname{Sin} \pi \lambda_{\beta m} \eta}{\operatorname{Sin} \frac{\pi}{2} \lambda_{\beta m}}-\frac{\operatorname{Sin} \pi \lambda_{\beta m}^{\prime} \eta}{\operatorname{Sin} \frac{\pi}{2} \lambda_{\beta m}^{\prime}}=\frac{8 \mu^{\prime}}{\pi} \sum_{n}(-1)^{\frac{n}{2}} \frac{n}{\left(n^{2}+\lambda_{\beta m}^{2}\right)\left(n^{2}+\lambda_{\beta m}^{\prime 2}\right)} \sin n \pi \eta, \\
& \frac{\lambda_{\beta m}^{2} \operatorname{Sin} \pi \lambda_{\beta m} \eta}{\operatorname{Sin} \frac{\pi}{2} \lambda_{\beta m}}-\frac{\lambda_{\beta m}^{2} \operatorname{Sin} \pi \lambda_{\beta m}^{\prime} \eta}{\operatorname{Sin} \frac{\pi}{2} \lambda_{\beta m}^{\prime}}=-\frac{8 \mu^{\prime}}{\pi} \sum_{n}(-1)^{\frac{n}{2}} \frac{n^{3}}{\left(n^{2}+\lambda_{\beta m}^{2}\right)\left(n^{2}+\lambda_{\beta m}^{\prime 2}\right)} \sin n \pi \eta \\
& (n=2,4,6 \ldots)
\end{aligned}
$$


kann man die Funktion $V_{\beta m}(\eta)$ in eine Fouriersche Reihe nach $\sin n \pi \eta$ entwickeln. Also nach einigen $Z$ wischenrechnungen wird

$$
V_{\beta m}(\eta)=\frac{\delta \mu^{\prime}}{\pi} \sum_{n}(-1)^{\frac{n}{2}} m n \frac{(1-\nu)^{2} m^{2} n^{2}+(2-\nu) \alpha^{2} \mu^{\prime 2}}{\left(n^{2}+\lambda_{s m}^{2}\right)\left(n^{2}+\lambda_{\beta m}^{\prime 2}\right)} \sin n \pi \eta .
$$

Die vorliegende Gleichung (a) kann nun gemäss $(c)$ und $(d)$ auf die Form

$$
\sum_{n}(-1)^{\frac{n}{2}}\left\{a_{n}^{\prime} H_{\alpha_{n}}+\frac{8 \mu^{\prime}}{\pi} \sum_{m} b_{m}^{\prime} \frac{m n\left[(1-\nu)^{2} m^{2} n^{2}+(2-\nu) a^{2} \mu^{\prime 2}\right]}{\left(n^{2}+\lambda_{i m}^{2}\right)\left(n^{2}+\lambda_{k m}^{\prime 2}\right)}\right\} \sin n \pi \eta=0
$$

gebracht werden. Daraus erhält man

$$
\begin{array}{r}
a_{n}^{\prime}+\frac{8 \mu^{\prime}}{\pi \cdot H_{\alpha n}} \sum_{r} b_{r}^{\prime} \frac{r n\left\{(1-\nu)^{2} r^{2} n^{2}+(2-\nu) \alpha^{2} \mu^{\prime 2}\right\}}{\left(n^{2}+\lambda_{\beta r}^{2}\right)\left(n^{2}+\lambda_{\beta r}^{\prime 2}\right)}=0 \\
(r, n=2,4,6 \ldots)
\end{array}
$$

mit

$$
H_{\alpha_{n}} \equiv \lambda_{\alpha_{n}}\left(\lambda_{\alpha_{n}}^{\prime 2}-\nu \alpha^{2} n^{2}\right)^{2} \Subset \operatorname{tg} \frac{\pi}{2} \lambda_{\alpha_{n}}-\lambda_{\alpha_{n}}^{\prime}\left(\lambda_{\alpha_{n}}^{2}-\nu \alpha^{2} n^{2}\right)^{2} \circlearrowleft \operatorname{tg} \frac{\pi}{2} \lambda_{\alpha_{n}}^{\prime}
$$

In analoger Weise ergibt sich aus den Randbedingungen 4)

mit

$$
\begin{array}{r}
b_{m}^{\prime}+\frac{8 \mu}{\pi \Pi_{\beta}} \sum_{s} a_{s}^{\prime} \frac{m s\left\{(1-\nu)^{2} m^{2} s^{2}+(2-\nu) \beta^{2} \mu^{2}\right\}}{\left(m^{2}+\lambda_{\alpha_{s}}^{2}\right)\left(m^{2}+\lambda_{\alpha_{s}}^{\prime 2}\right)}=0 \\
(m, s=2,4,6 \ldots)
\end{array}
$$

$$
\left.H_{\beta m} \equiv \lambda_{\beta m}\left(\lambda_{\beta m}^{\prime 2}-\nu \beta^{2} m^{2}\right)^{2} \operatorname{ctg} \frac{\pi}{2} \lambda_{\beta m}-\lambda_{\beta m}^{\prime}\left(\lambda_{i m}^{2}-\nu \beta^{2} m^{2}\right)^{2} \operatorname{ctg} \frac{\pi}{2} \lambda_{\beta m}^{\prime} \cdot\right\}
$$

Vermöge der Formeln (29) und (30) kann man weiter aus (29) die unbekannten Beiwerte $b_{m}^{\prime}$ eliminieren, wie folgt:

$$
a_{n}^{\prime}+\sum_{s} K_{n s} a_{s}^{\prime}=0
$$

mit

$$
\left.K_{n s}=-\frac{64 \mu \mu^{\prime} n s}{\pi^{2} H_{\alpha_{n}}} \sum_{r} \frac{r^{2}\left\{(1-\nu)^{2} r^{2} n^{2}+(2-\nu) a^{2} \mu^{\prime 2}\right\}\left\{(1-\nu)^{2} r^{2} s^{2}+(2-\nu) \beta^{2} \mu^{2}\right\}}{U_{i r}\left(n^{2}+\lambda_{\beta r}^{2}\right)\left(n^{2}+\lambda_{\beta r}^{\prime 2}\right)\left(r^{2}+\lambda_{\alpha s}^{2}\right)\left(r^{2}+\lambda_{\alpha s}^{\prime 2}\right)} \cdot \underset{\left(r^{2}, n, s=2,4,6, \ldots\right)}{ }\right\}
$$

Dieser Ausdruck für $K_{n s}$ unterscheidet sich von $K_{n s}(14)$ formal nur durch $H_{a n}$ und $H_{\beta}$, and die Frequenzgleichung für diesen Fall wird analog wie (15) durch

ausgedrückt.

$$
\Delta=\left|\begin{array}{rrrrr}
1+K_{22} & K_{24} & K_{26} & K_{28} & \ldots \\
K_{42} & 1+K_{44} & K_{46} & K_{48} & \ldots \\
K_{62} & K_{64} & 1+K_{66} & K_{68} & \ldots \\
K_{82} & K_{84} & K_{86} & 1+K_{88} & \ldots \\
\ldots & \ldots & \ldots & \ldots & \ldots
\end{array}\right|=0
$$


8. Die Formeln und die numerischen Rechenergebnisse für die quadra. tische Platte. Wegen $m=n=2,4,6 \ldots, \alpha=\beta=1, \mu^{\prime}=\mu$ für die quadratische Platte kann man hierfür schreiben

$$
\begin{gathered}
w^{\prime}=\sum_{n}(-1)^{\frac{n}{2}}\left\{a_{n}^{\prime} v_{n}(\xi) \sin n \pi \eta+b_{n}^{\prime} v_{n}(\eta) \sin n \pi \xi\right\} . \\
(n=2,4,6 \ldots)
\end{gathered}
$$

Der Ausdruck für $K_{n s}$ lautet nach (31)

mit

$$
\left.\begin{array}{c}
K_{n s}=-\frac{64 \mu^{2} n s}{\pi^{2} H_{n}} \sum_{r} \frac{r^{2}\left\{(1-\nu)^{2} \gamma^{2} n^{2}+(2-\nu) \mu^{2}\right\}\left\{(1-\nu)^{2} r^{2} s^{2}+(2-\nu) \mu^{2}\right\}}{\Pi_{r}\left\{\left(r^{2}+n^{2}\right)^{2}-\mu^{2}\right\}\left\{\left(r^{2}+s^{2}\right)^{2}-\mu^{2}\right\}} \\
(r, n, s=2,4,6 \ldots) \\
H_{n} \equiv \lambda_{n}\left(\lambda_{n}^{\prime 2}-\nu n^{2}\right)^{2}\left(5 \operatorname{tg} \frac{\pi}{2} \lambda_{n}-\lambda_{n}^{\prime}\left(\lambda_{n}^{2}-\nu n^{2}\right)^{2} \operatorname{ctg} \frac{\pi}{2} \lambda_{n}^{\prime}\right.
\end{array}\right\}
$$

Der Ausdruck für $H_{r}$ ist derselbe wie der für $H_{n}$ mit $r$ an Stelle von $n$. Auch für diesen Fall wie für die erste Familie der ersten Schwingungsart ist es möglich, die Frequenzgleichung: (32) mit $K_{n s}$ (34) in zwei verschiedene Gleichungen von der analogen Form wie (19) und (20) zu zerlegen.

1) Die erste Schwingungsfamilie. Wir wollen jetzt die erste Familie der zweiten Schwingungsart betrachten, in der die Platte antisymmetrisch in bezug auf die beiden Mittellinjen, symmetrisch dagegen in bezug auf die beiden Diagonalen des Quadrats schwingt. Hierbei hat die Schwingungsfläche mindestens zwei Knotenlinien $\xi=0$ und $\eta=0$ und wird wegen $b_{n}^{\prime}=a_{n}^{\prime}$ durch

$$
\begin{aligned}
& w^{\prime}=a_{2} \bar{w}^{\prime} \\
& \bar{w}^{\prime}=\sum_{n}(-1)^{\frac{n}{2}} \alpha_{n}^{\prime}\left\{v_{n}(\xi) \sin n \pi \eta+v_{n}(\eta) \sin n \pi \xi\right\} \\
& \quad \alpha_{n}^{\prime}=\frac{a_{n}^{\prime}}{a_{2}}, \quad\left(\alpha_{2}^{\prime}=1\right), \quad(n=2,4,1 \ldots)
\end{aligned}
$$

ausgedrückt. Dafür gilt aus (20) die Bedingungsgleichung

$$
a_{n}^{\prime}+\frac{8 \mu}{\pi H_{n}} \sum_{s} a_{s}^{\prime} \frac{n s\left\{(1-\nu)^{2} n^{2} s^{2}+(2-\nu) \mu^{2}\right\}}{\left(n^{2}+s^{2}\right)^{2}-\mu^{2}}=0
$$

oder in anderer Schreibweise

mit

$$
\left.\begin{array}{l}
a_{n}^{\prime}+\sum_{s} K_{n s} a_{s}^{\prime}=0, \quad(n, s=2,4,6 \ldots) \\
K_{n s}=\frac{8 \mu n s}{\pi H_{n}} \frac{(1-\nu)^{2} n^{2} s^{2}+(2-\nu) \mu^{2}}{\left(n^{2}+s^{2}\right)^{2}-\mu^{2}}
\end{array}\right\}
$$


Man kann beweisen, dass dié durch $K_{n s}$ (36) gebildete Frequenzgleichung auch aus der Determinantengleichung (32) mit $K_{n s}$ (34) hervorgeht.

In analoger Weise wie in den früheren Fällen erhält man die in Tabelle 4 zusammengestellten Rechenergebnisse für die erste und zweite Wurzel $\mu$.

Tabelle 4. Die Werte von $\mu$ und die der verschiedenen Beiwerte für die erste Familie der zweiten Schwingungsart der quadratischen Platte mit $\nu=0,3$.

\begin{tabular}{|c|c|c|c|c|c|c|c|}
\hline 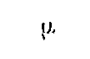 & $n$ & $\alpha_{n}$ & $\lambda_{n}$ & $\gamma^{\prime 2} n-v n^{2}$ & $\ell_{n}^{\prime}$ & $\lambda^{2}{ }_{n}-v n^{2}$ & $\begin{array}{c}\text { Abb. } 8 . \\
\text { Knotenlinien. }\end{array}$ \\
\hline \multirow{4}{*}{ 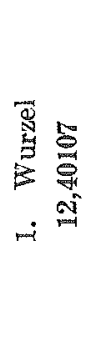 } & 2 & 1,000000 & 4,049823 & $-9,60107$ & $2,898459 i$ & 15,20107 & \\
\hline & 4 & 0,368251 & 5,329265 & $-1,20 \mathrm{IC7}$ & 1,897084 & 23,60107 & \\
\hline & 6 & 0,026749 & 6,957087 & 12,79893 & 4,587875 & 37,60107 & \\
\hline & 8 & 0,006794 & 8,740770 & 32,39893 & 7,183239 & 57,20107 & (1) \\
\hline \multirow{4}{*}{ 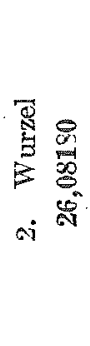 } & 2 & 1,000000 & $5,484,688$ & $-23,28180$ & $4,698127 i$ & 28,88180 & \\
\hline & 4 & 0,303269 & 6,487048 & $-14,88180$ & $3,175181 i$ & 37,28180 & \\
\hline & 6 & 0,194748 & 7,879201 & $-0,88180$ & 3,149318 & 51,28180 & \\
\hline & 8 & 0,025757 & 9,491144 & 18,71820 & 6,157775 & 70,88180 & (II) \\
\hline
\end{tabular}

Abb. 9-(I) bzw. 9-(II) stellt die Schichtlinien der $\bar{w}-F l a ̆ c h e$ für die erste Wurzel $\mu_{1}=12,40107$ bzw , die zweite $\mu_{2}=26,08180$ dar.

2) Die zweite Schwingungsfamilie. Es handelt sich jetzt um die zweite Familie der zweiten Schwingungsart, in welcher die Schwingungstläche der Platte sich antisymmetrisch nicht nur in bezug auf die beiden Mittellinien, sondern auch auf die beiden Diagonalen des Quadrats gestaltet. Hierbei hat die Schwingungsfläche mindestens vier Knotenlinien $\xi=0, \eta=0, \xi=\eta, \xi=-\eta$, und wird nur durch Umkehrung des Vorzeichens für $v_{n}(\eta)$ in (35) ausgedrüickt. Also lautet

$$
\begin{aligned}
& \bar{w}^{\prime}=\sum_{n}(-1)^{\frac{n}{2}} \alpha_{n}^{\prime}\left\{v_{n}(\xi) \sin n \pi \eta-v_{n}(\eta) \sin n \pi \xi\right\} \\
& \alpha_{n}^{\prime}=\frac{a_{n}}{a_{2}}, \quad\left(\alpha_{2}=1\right), \quad(n=2,4,6 \ldots) .
\end{aligned}
$$




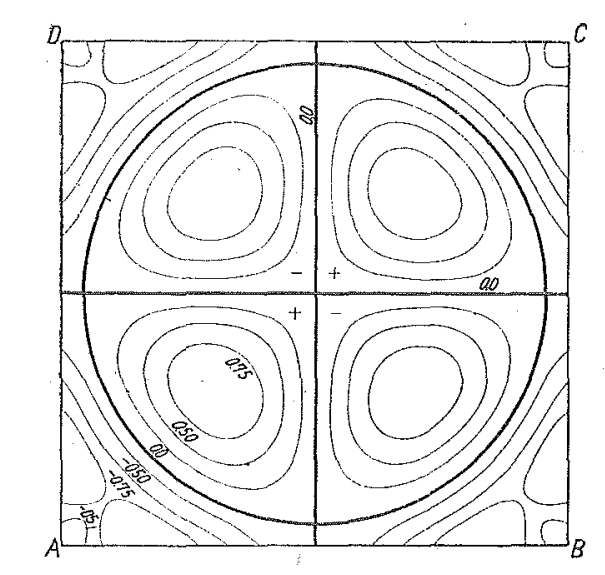

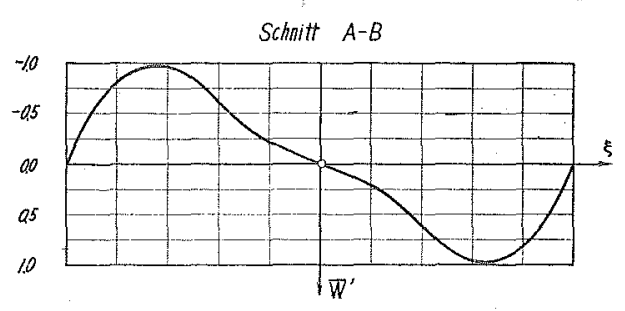

(I). $\mu_{1}=12,40107$.
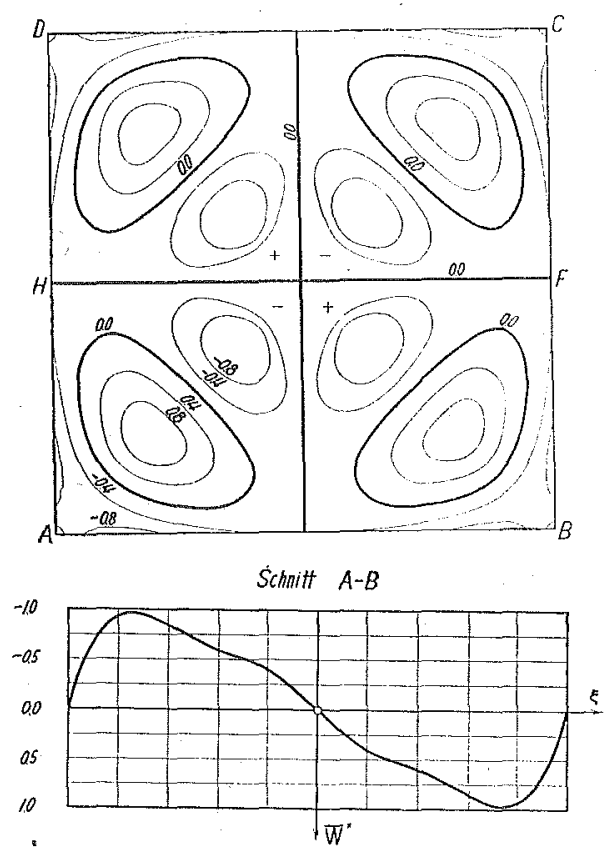

(II . $\mu_{2}=26,08180$.

Abb. 9. Schichtlinien der $\bar{u}^{\prime}$-Fläche für die erste Familie der zweiten Schwingungsart.

Analog ist der Ausdruck für $K_{n \varepsilon}$ derselbe, wie der in (36) mit entgegengesetztem Vorzeichen.

Tabelle 5. Die Werte der verschiedenen. Beiwerte für die zweite Familie der zweiten Schwingungsart der quadratischen Platte mit $\nu=0,3$.

\begin{tabular}{|c|c|c|c|c|c|c|c|}
\hline$\mu$ & $n$ & $\alpha_{n}$ & $\lambda_{n}$. & $x^{\prime 2} n-y_{n}^{2}$ & $z_{n}^{\prime}$ & $\lambda^{2}{ }_{n}-v n^{2}$ & $\begin{array}{c}\text { Abb. } 10 . \\
\text { Knotenlinien. }\end{array}$ \\
\hline \multirow{4}{*}{ 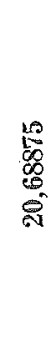 } & 2 & 1,000000 & 4,968777 & $-17,88875$ & $4,085187 i$ & 23,48875 & \\
\hline & 4 & 1,284942 & 6,057125 & $-9,48875$ & $2,165352 i$ & 31,88875 & \\
\hline & 6 & $-0,131509$ & 7,529193 & 4,51125 & 3,912959 & 45,88875 & \\
\hline & 8 & $-0,023564$ & 9,202649 & 24,11125 & 6,581128 & 65,48875 & \\
\hline
\end{tabular}


Die Rechenergebnisse für die erste Wurzel $\mu=20,68875$ sind in Tabelle 5 zusammengestellt, während die schematisch gezeichneten Knotenlinien dafür bzw. die entsprechenden Schichtlinien der $\bar{w}^{\prime}$-Fläche durch Abb. 10 bzw. 11 dargestellt werden.

\section{Die dritte Schwingungsart.}

9. Die allgemeinen Formeln für die rechteckige Platte. In den früheren Untersuchungen sieht man, dass die Platte in der ersten sowie der zweiten Schwingungsart immer derart schwingt, dass die Auslenkung einer beliebig durch den Mittelpunkt des Quadrats gezogenen geraden Linie symmetrisch in hezug auf diesen Punkt ist. Wir gehen jetzt dazu über, den anderen Schwingungsfall zu betrachten, in dem irgendeine solche

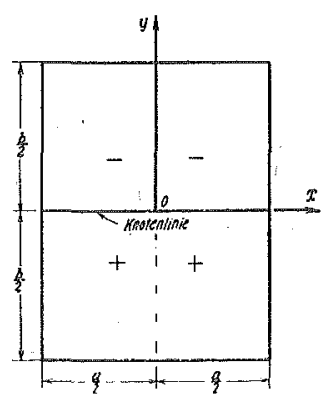

Abb. 12. $w_{a}^{\prime}-$ Fläche.

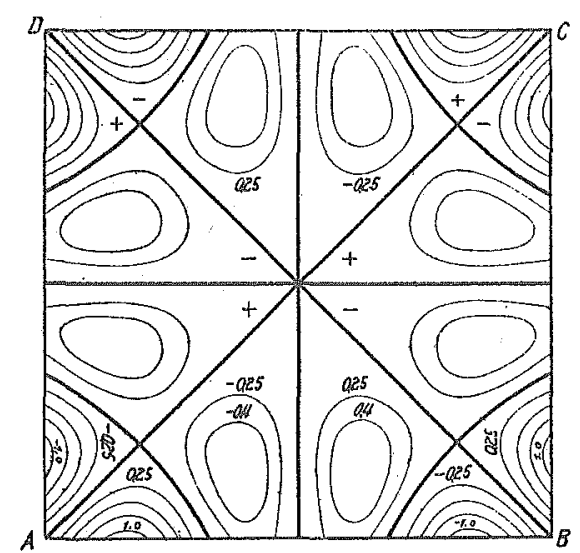

Schaitt $A-B$

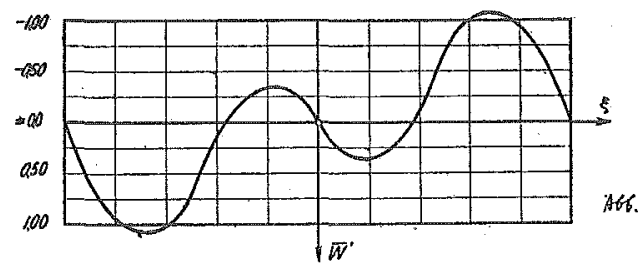

Abb. 11. Schichtlinien der $\bar{u}^{\prime}-$ Fläche für die zweite Familie der zweiten Schwingungsart mit $\mu=20,68875$. metrisch in bezug auf ihren Mittelpunkt schwingt.

Wenn nun die Schwingungsfläche symmetrisch in bezug auf die Mittellinie $\xi=0$, antisymmetrisch dagegen in bezug auf die andere $\eta=0$ ist, so muss sie mindestens eine Knotenlinie $\eta=0$ haben, wie $\mathrm{Abb} .12$ zeigt, und die schwingende elastische Linie einer beliebig durch den Mittelpunkt des Quadrats gezogegen geraden Linie verläuft antisymmetrisch in bezug auf diesen Punkt. Eine Schwingungsfläche $w_{a}^{\prime}$ mit dieser Eigenschaft wird durch

$$
\begin{gathered}
w_{a}^{\prime}=\sum_{n}(-1)^{\frac{n}{2}} a_{n} u_{\alpha_{n}}(\xi) \sin n \pi \eta+\sum_{m}(-1)^{\frac{m-1}{2}} b_{m}^{\prime} v_{\beta m}(\eta) \cos m \pi \xi \\
(m=1,3,5 \ldots, n=2,4,6 \ldots)
\end{gathered}
$$

mit

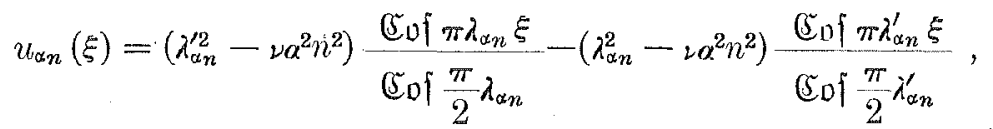

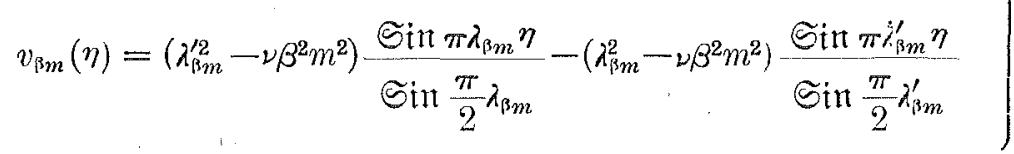


ausgedrïckt, Die beiden unbekannten Beiwerte $a_{n}$ und $b_{m}^{\prime}$ werden durch die aus den Randbedingungen 3) bzw. 4) hervorgehenden Gleichungen

$$
\begin{gathered}
\sum_{n}(-1)^{\frac{n}{2}} a_{n}\left\{\frac{a^{3}}{\pi^{3}} u_{\alpha n}^{\prime \prime \prime}\left( \pm \frac{1}{2}\right)-\alpha^{2} n^{2}(2-\nu) \frac{a}{\pi} u_{\alpha n}^{\prime}\left( \pm \frac{1}{2}\right)\right\} \sin n \pi \eta \\
\pm \sum_{m} b_{m}^{\prime}\left\{m^{3} v_{\beta m}(\eta)-\alpha^{2} m(2-\nu) \frac{b^{2}}{\pi^{2}} v_{\beta m}^{\prime \prime}(\eta)\right\}=0
\end{gathered}
$$

bzw.

$$
\begin{gathered}
\sum_{m}(-1)^{\frac{m-1}{2}} b_{m}^{\prime}\left\{\frac{b^{3}}{\pi^{3}} v_{\beta m}^{\prime \prime \prime}\left( \pm \frac{1}{2}\right)-\beta^{2} m^{2}(2-\nu) \frac{b}{\pi} v_{\beta m}^{\prime}\left( \pm \frac{1}{2}\right)\right\} \cos m \pi \xi \\
-\sum_{n} a_{n}\left\{n^{3} u_{\alpha_{n}}(\xi)-\beta^{2} n(2-\nu) \frac{a^{2}}{\pi^{2}} u_{\alpha_{n}}^{\prime \prime}(\xi)\right\}=0
\end{gathered}
$$

miteinander verknüpft: Mit Rücksicht auf $(c)$ auf Seite 324 sowie $(d)$ auf Seite 337 erhält man

$$
\begin{array}{r}
a_{n}+\frac{8 \mu^{\prime}}{\pi G_{\alpha n}} \sum_{r} b_{r}^{\prime} \frac{r n\left\{(1-\nu)^{2} r^{2} n^{2}+(2-\nu) \alpha^{2} \mu^{\prime 2}\right\}}{\left(n^{2}+\lambda_{s r}^{2}\right)\left(n^{2}+\lambda_{i r}^{\prime 2}\right)}=0, \\
(r=1,3,5 \ldots, n=2,4,6 \ldots) \\
b_{m}^{\prime}+\frac{8 \mu}{\pi I_{i m}} \sum_{s} a_{s} \frac{m s\left\{(1-\nu)^{2} m^{2} s^{2}+(2-\nu) \beta^{2} \mu^{2}\right\}}{\left(m^{2}+\lambda_{\alpha_{s}}^{2}\right)\left(m^{2}+\lambda_{\alpha s}^{\prime 2}\right)}=0 . \\
(m=1,3,5 \ldots, \quad s=2,4,6 \ldots)
\end{array}
$$

In den vorangehenden Gleichungen ist der Ausdruck für $G_{\alpha n}$ bzw. $H_{\beta m}$ derselbe wie der in (12) bzw. (30). Wenn man ferner den unmittelbar aus (40) ermittelten Ausdruck für $b_{r}^{\prime}\left(=b_{m}^{\prime}\right)$ in (39) einsetzt, so erhält man wie immer

$$
a_{n}+\sum_{s} K_{n s} a_{s}=0
$$

mit

$$
\left.\begin{array}{c}
K_{n s}=-\frac{\left(64 \mu \mu^{\prime} n s\right.}{\pi^{2} G_{\alpha n}} \sum_{r} \frac{r^{2}\left\{(1-\nu)^{2} r^{2} n^{2}+(2-\nu) \alpha^{2} \mu^{\prime 2}\right\}\left\{(1-\nu)^{2} r^{2} s^{2}+(2-\nu) \beta^{2} \mu^{2}\right\}}{H_{\beta r}\left(n^{2}+\lambda_{\beta r}^{2}\right)\left(n^{2}+\lambda_{\beta r}^{\prime 2}\right)\left(r^{2}+\lambda_{\alpha s}^{2}\right)\left(r^{2}+\lambda_{\alpha s}^{\prime 2}\right)} \cdot \\
\left(r^{2}=1,3,5 \ldots, n, s=2,4,6 \ldots\right)
\end{array}\right\}
$$

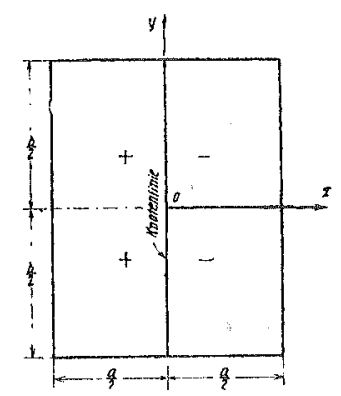

Abb. 13.

$w b^{\prime}$-Fläche.
Daraus kann man leicht die Frequenzgleichung herstellen.

Wenn es sich weiter um den Fall handelt, in dem die Schwingungsfläche antisymmetrisch bzw. symmetrisch in bezug auf die Mittellinie $\xi=0$ bzw. $\eta=0$ des Rechtecks ist, wie Abb. 13 zeigt, so ergibt sich ohne weiteres 


$$
\begin{gathered}
w_{b}^{\prime}=\sum_{n}(-1)^{\frac{n-1}{2}} a_{n}^{\prime} v_{a n}(\xi) \cos n \pi \eta+\sum_{m}(-1)^{\frac{m}{2}} b_{m} u_{\beta m}(\eta) \sin m \pi \xi \\
(m=2,4,6 \ldots, \quad n=1,3,5 \ldots)
\end{gathered}
$$

Dafür gilt offenbar

mit

$$
b_{m}+\sum_{r} K_{m r} b_{r}=0
$$

$$
\left.\begin{array}{c}
K_{m r}=-\frac{64 \mu \mu^{\prime} m r}{\pi^{2} G_{\beta m}^{g}} \sum_{s} \frac{s^{2}\left\{(1-\nu)^{2} m^{2} s^{2}+(2-\nu) \beta^{2} \mu^{2}\right\}\left\{(1-\nu)^{2} r^{2} s^{2}+(2-\nu) \alpha^{2} \mu^{\prime 2}\right\}}{H_{\alpha_{s}}\left(m^{2}+\lambda_{\alpha_{s}}^{2}\right)\left(m^{2}+\lambda_{\alpha_{s}}^{\prime 2}\right)\left(s^{2}+\lambda_{\beta r}^{2}\right)\left(s^{2}+\lambda_{\beta r}^{\prime 2}\right)} . \\
(m, r=2,4,6 \ldots, \quad s=1,3,5 \ldots)
\end{array}\right\}
$$

Wenn nun die beiden Funktionen $w_{a}^{\prime}$ and $w_{b}^{\prime}$ so verbunden werden, dass

$$
w=A w_{a}+B w_{b}=A w_{a}^{\prime} \cos \omega_{a} t+B w_{b}^{\prime} \cos \omega_{b} t,
$$

wo $A$ und $B$ beliebige reelle Zahlen bedeuten, führt $w$ nur dann eine periodische Schwingung aus, wenn das Verhältnis der beiden Schwingungsdauern

eine rationale Zahl ist.

$$
\frac{T_{a}}{T_{b}^{\prime}}=\frac{\omega_{b}}{\omega_{a}}=\frac{\mu_{b}}{\mu_{a}}
$$

10. Die Formeln und die numerischen Rechenergebnisse für die quadratische Platte. Die Formeln fir die dritte Schwingungsart der quadratischen Platte kann man leicht unmittelbar aus (33) bis (43) mit $\alpha=\beta=1, \mu=\mu^{\prime}$ ableiten. Da aber der Ausdruck fir $K_{n s}$ oder $K_{m r}$ immer durch die Summe der unendlichen Reihe ausgedrückt wird, so sind die numerischen Rechenverfahren viel mühsamer als die für die erste sowie für die zweite Schwingungsart.

1) Die erste Schwingungsfamilie. Wir wollen jetzt den Fall betrachten, in dem die Platte symmetrisch in bezug auf die Mittellinie $\xi=0$, antisymmetrisch dagegen in bezug auf die andere $\eta=0$ des Quadrats schwingt. Die Schwingungsfläche hierfür lautet sofort nach (38), wie folgt:

$$
\begin{gathered}
w_{a}^{\prime}=a_{2} \bar{w}_{a}^{\prime}, \\
\bar{w}_{a}^{\prime}=\sum_{n}(-1)^{\frac{n}{2}} \alpha_{n} u_{n}(\xi) \sin n \pi \eta+\sum_{m}(-1)^{\frac{m-1}{2}} \beta_{m}^{\prime} v_{m}(\eta) \cos m \pi \xi \\
(m=1,3,5 \ldots, \quad n=2,4,6 \ldots) \\
\alpha_{n}=\frac{a_{n}}{a_{2}}, \quad\left(\alpha_{2}=1\right), \quad \beta_{m}^{\prime}=\frac{b_{m}^{\prime}}{a_{2}} .
\end{gathered}
$$

Dafür gilt

$$
\left.\begin{array}{l}
\alpha_{n}+\sum_{s} K_{n s} \alpha_{s}=0, \\
K_{n s}=-\frac{64 \mu^{2}}{\pi^{2} G_{n}} n s \sum_{r} \frac{r^{2}\left\{(1-\nu)^{2} r^{2} n^{2}+(2-\nu) \mu^{2}\right\}\left\{(1-\nu)^{2} r^{2} s^{2}+(2-\nu) \mu^{2}\right\}}{H_{r}\left\{\left(n^{2}+r^{2}\right)^{2}-\mu^{2}\right\}\left\{\left(r^{2}+s^{2}\right)^{2}-\mu^{2}\right\}} \\
(r=1,3,5 \ldots, \quad n, s=2,4,6 \ldots)
\end{array}\right\}
$$


mit

$$
\begin{aligned}
& G_{n} \equiv \lambda_{n}\left(\lambda_{n}^{\prime 2}-\nu n^{2}\right)^{2} \mathfrak{I g} \frac{\pi}{2} \lambda_{n}-\lambda_{n}^{\prime}\left(\lambda_{n}^{2}-\nu n^{2}\right)^{2} \mathfrak{T} \mathfrak{q} \frac{\pi}{2} \lambda_{n}^{\prime}, \\
& H_{r} \equiv \lambda_{r} \cdot\left(\lambda_{r}^{\prime 2}-\nu r^{2}\right)^{2}\left(\check{t g} \frac{\pi}{2} \lambda_{r}-\lambda_{r}^{\prime}\left(\lambda_{r}^{2}-\nu r^{2}\right)^{2}\left(5 \operatorname{tg} \frac{\pi}{2} \lambda_{r}^{\prime} .\right.\right.
\end{aligned}
$$

Wenn man daraus die Werte von $\alpha_{n}$ ausrechnet, so kann man die Werte von $\beta_{m}^{\prime}$ aus der aus (40) hervorgehenden Gleichung

$$
\beta_{m}^{\prime}\left(=\frac{b_{m}^{\prime}}{a_{2}}\right)=-\frac{8 \mu}{\pi H_{m}} \sum_{s} \alpha_{s} \frac{m s\left\{(1-\nu)^{2} m^{2} s^{2}+(2-\nu) \mu^{2}\right\}}{\left(m^{2}+s^{2}\right)^{2}-\mu^{2}}
$$

ermitteln.

Analog für die antisymmetrisch bzw. symmertisch in bezug auf $\xi=0$ bzw. $\eta=0$ schwingende Fläche ergibt sich aus (42)

$$
\begin{gathered}
w_{b}^{\prime}=b_{2} \bar{w}_{b}^{\prime}, \\
\bar{w}_{b}^{\prime}=\sum_{n}(-1)^{\frac{n-1}{2}} \alpha_{n}^{\prime} v_{n}(\xi) \cos n \pi \eta+\sum_{m}(-1)^{\frac{m}{2}} \beta_{m} u_{m}(\eta) \sin m \pi \xi, \\
(m=2,4,6 \ldots, \quad n=1,3,5 \ldots), \\
\alpha_{n}^{\prime}=\frac{a_{n}^{\prime}}{b_{2}}, \quad \beta_{m}=\frac{b_{m}}{b_{2}}, \quad\left(\beta_{2}=1\right) .
\end{gathered}
$$

Es ist leicht ersichtlich, dass die eine von den Flächen $\bar{w}_{a}^{\prime}$ und $\bar{w}_{b}^{\prime}$ durch eine Drehung um $90^{\circ} \mathrm{um}$ den Mittelpunkt des Quadrats mit der anderen identisch wird. Somit müssen die Grössen von $\mu$ für $\bar{w}_{b}^{\prime}$ gleich den entsprechenden Grössen von $\mu$ für $\bar{w}_{a}^{\prime}$ sein. Die Werte von $\mu$ für die beiden Schwingungs-

\begin{tabular}{|c|c|c|c|c|c|c|}
\hline$\mu$. & $n$ & $\alpha_{n}$ & $\lambda_{n}$ & $\lambda^{\prime 2} n-n^{2}$ & $x_{n}^{\prime}$ & $\lambda_{n}^{2} n_{n}-v n^{2}$ \\
\hline \multirow{4}{*}{ 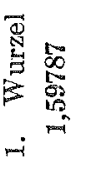 } & 2 & 1,000000 & 2,365982 & 1,20213 & 1,549880 & 4,39787 \\
\hline & 4 & 0,034765 & 4,194982 & 9,60213 & 3,795013 & 12,79787 \\
\hline & 6 & 0,006040 & 6,131710 & 23,60213 & 5,865334 & 26,79787 \\
\hline & 8 & 0,001841 & 8,099252 & 43,20213 & 7,899502 & 46,39787 \\
\hline \multirow{4}{*}{ 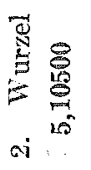 } & 2 & 1,000000 & 3,017449 & $-2,30500$ & $1,051190 i$ & 7,90500 \\
\hline & 4 & $-0,003879$ & 4,594017 & 6,09500 & 3,300757 & 16,30500 \\
\hline & 6 & 0,000530 & 6,411318 & 20,09500 & 5,558321 & 30,30500 \\
\hline & 8 & 0,000358 & 8,212941 & 39,69500 & 7,674307 & 49,90500 \\
\hline
\end{tabular}
flächen können daher durch die Auflösung der durch $K_{n s}$ (45) gebildeten Frequenzgleichung ermittelt werden. Die wirkliche Durchrechnung für die ersten zwei Eigenwerte liefert die in Tabelle 6 zusammengestellten Ergebnisse.

Tabelle 6 . Die Werte von $\mu$ und die der versehiedenen Beiwerte für die dritte Schwingungsart der quadratischen

Platte mit $\nu=0,3$. 


\begin{tabular}{|c|c|c|c|c|c|c|}
\hline$\mu$. & $m$ & $\beta_{m}^{\prime}$ & $\lambda_{m}$ & $\lambda^{\prime 2}{ }_{m}-v m^{2}$ & $\cdots \lambda_{m}^{\prime}$ & $\lambda .^{2}{ }^{2}-i m^{2}$ \\
\hline \multirow{4}{*}{ 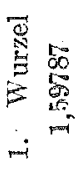 } & 1 & $-11,369403$ & 1,611791 & $-0,89787$ & $0,773221 i$ & 2,29787 \\
\hline & 3 & $-0,035521$ & 3,255437 & 4,70213 & 2,720685 & 7,89787 \\
\hline & 5 & $-0,006377$ & 5,157313 & 15,90213 & 4,837575 & 19,09787 \\
\hline & 7 & $-0,001917$ & 7,113219 & 32,70213 & 6,884921 & 35,89787 \\
\hline \multirow{4}{*}{ 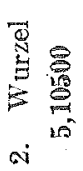 } & 1 & $-0,219366$ & 2,470830 & $-4,40500$ & $2,026080 i$ & 5,80500 \\
\hline & 3 & 0,041799 & 3,755663 & 1,19500 & 1,973575 & 11,40500 \\
\hline & 5 & 0,003222 & 5,486803 & 12,39500 & 4,460380 & 22,60500 \\
\hline & 7 & 0,001143 & 7,355610 & 29,19500 & 6,625331 & 39,40500 \\
\hline
\end{tabular}

1. Wurzel $\mu_{1}=1,59787$.

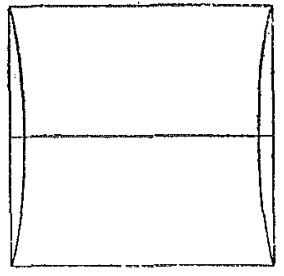

$(I-1), \kappa=0,0$.

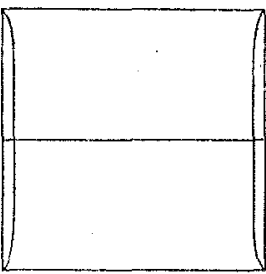

$(I-1), \kappa=0,0$.

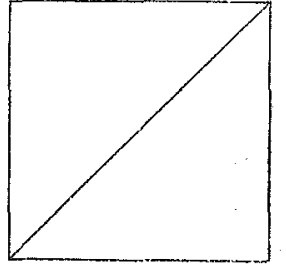

$(I-2), k=-1,0$.

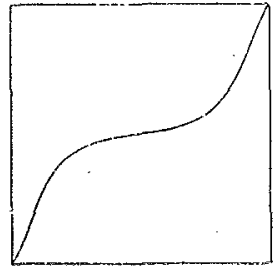

$(I-3), \kappa=-0,2$.

2. Wurzel $\mu_{2}=5,10500$.

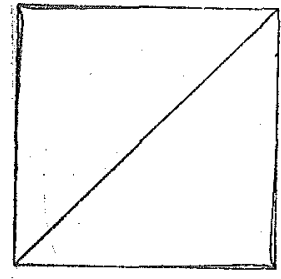

$(I I-2), \kappa=-1,0$.

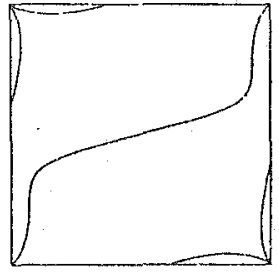

$(\mathrm{II}=3), \kappa=-0,3$.

Abb. 14. Schematische Darstellung der Knotenfiguren für die dritte Schwinungsart der quadratischen Platte mit $v=0,3$.

Abb. 1t-(I-1) bzw. (II-1) zeigt die schematisch gezeichneten Knotenlinien für die erste Wurzel $\mu_{1}=1,59787 \mathrm{bzw}$. die zweite $\mu_{2}=5,10500$, während die entsprechenden Schichtlinien der $\bar{w}_{a}$-Fläche durch Abl. 15 dargestellt werden.

Wie man aus Abb. $15-(\mathrm{I}-1)$ sieht, liegt jede der die Plattenecken passierenden Knotenlinien sehr nahe dem zur Knotenlinie $\eta=0$ senkrecht stehenden entsprechenden Plattenrande, dessen Auslenkung $\bar{w}_{a c}^{\prime}$ infolgedessen sehr klein wird. So schwingt die Platte hierbei, als ob sie auf den beiden Rändern $\xi= \pm \frac{1}{2}$ frei gestützt wird. Diese Tatsache ist deswegen ganz natürlich, weil die Grösse $\mu_{1}=1,59787$ für diesen Schwingunsfall sehr wenig von der ersten 

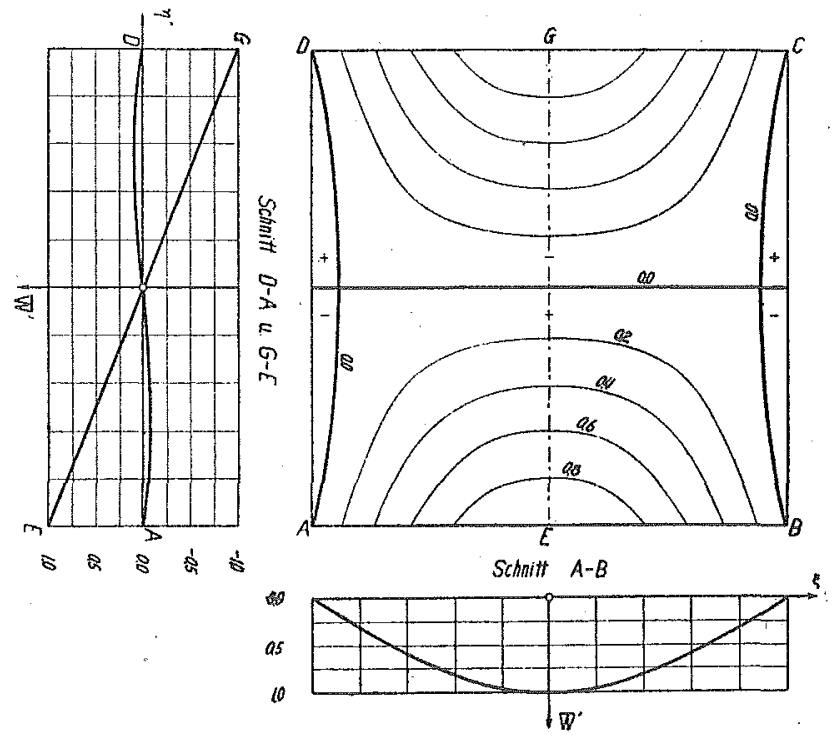

$(\mathrm{I}-1), \mu_{1}=1,69787(\kappa=0,0)$.
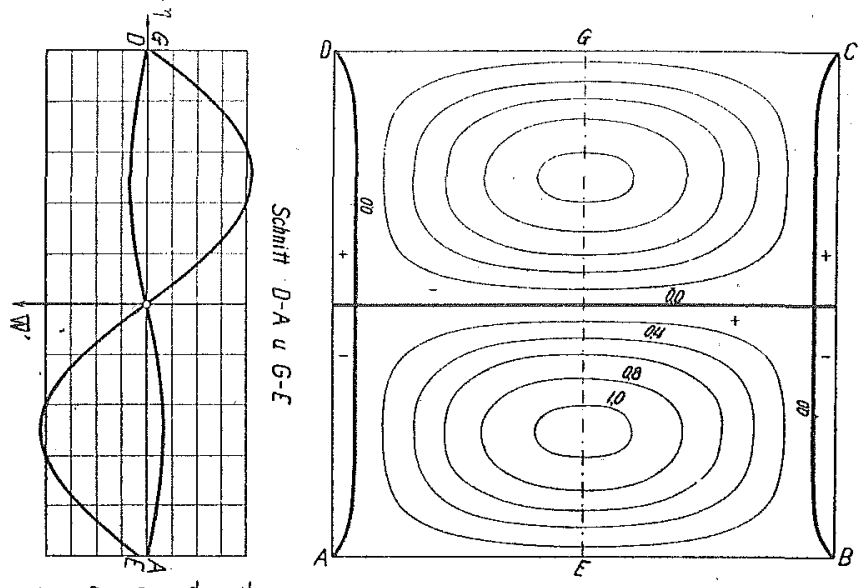

क 98 ह

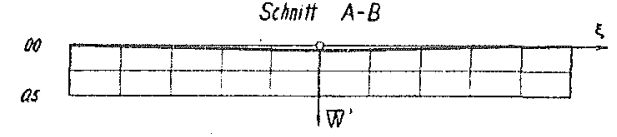

$(\mathrm{II}-1), \mu_{2}=5,10500(\kappa=0,0)$.

Abb. 15. Schichtlinien der $\bar{t}^{\prime} a^{\prime}-$ Fläche für die erste Familie der dritten Schwingnngsart.

$$
\bar{w}^{\prime}=\bar{w}_{a}^{\prime} \pm \bar{w}_{b}^{\prime}
$$

erfïllt. Diese Verbindung lst deswegen mög]ich, weil, wie schon erwähnt, jede Teilschwingung $\bar{w}_{a}^{\prime}$ und $\bar{w}_{b}^{\prime}$, und folglich auch die resultierende Schwingung $\bar{w}^{\prime}$

1) S. Iguchi, Doboku-Gakkai.Si, 26 (1940) S. 431.

2) S. Iguchi, Mem. Fac. Engng., Hokkaido Imp. Univ. 4 (1938) S. 322 ; Lord Rayleigh, "Theory of Sound," 1 S. 371, 1929. 
eine und dieselbe Schwingungsdauder, also die gleiche Tonhöhe hat.

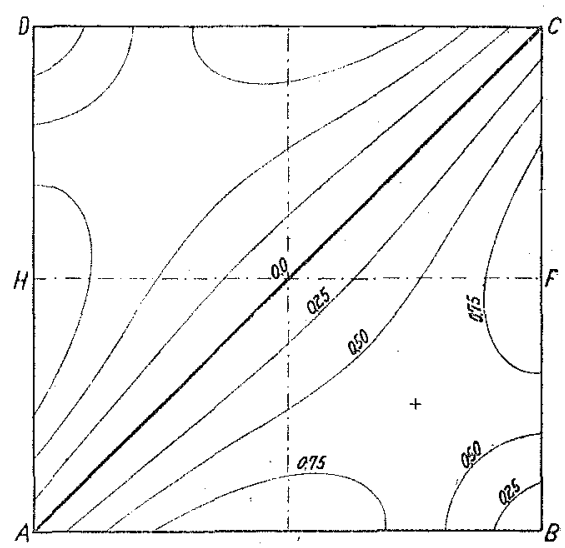

Schnitt $A-B$ u. H-F

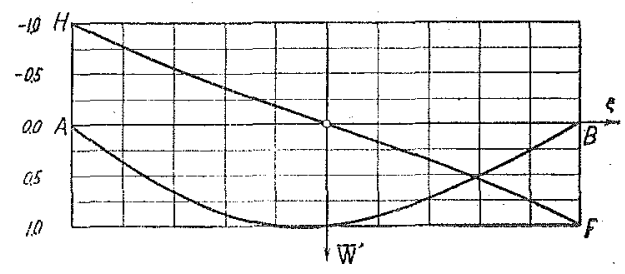

$(\mathrm{I}-2), \mu_{1}=1,59787(\kappa=-1,0)$.

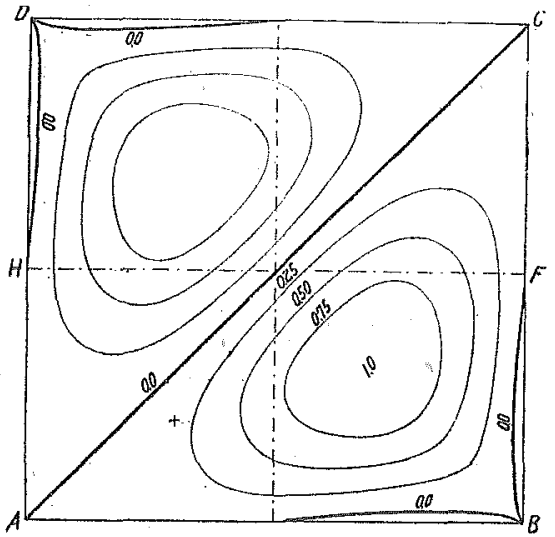

Schnitt A-B u. H-F

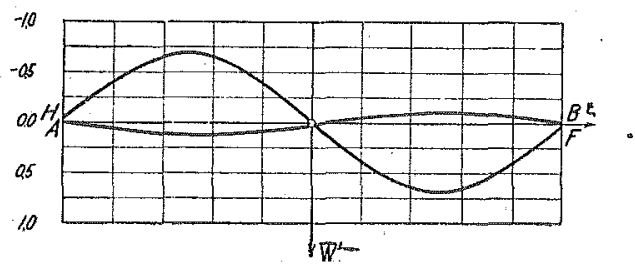

$\left(\mathrm{II}-2, \mu_{2}=5,10500(x=-1,0)\right.$.

Abb. 16. Schichtlinien der Fläche $\bar{w}^{\prime}=\bar{u}^{\prime} a^{\prime}-\bar{u}^{\prime} b^{\prime}$ für die zweite Familie der dritten Schwingungsart.

Die schematiseh gezeichnete Knotenfigur für die erste Wurzel $\mu_{1}=1,59787 \mathrm{bzw}$. die zweite $\mu_{2}=5,10500$ wird in Abb. 14 mit dem Zeichen (I-2) bzw. (II-2) gezeigt, während die entsprechenden Schichtlinien der Fläche $\bar{w}^{\prime}=\bar{u}_{a}^{\prime}-\bar{u}_{b}^{\prime}$ durch Abb. 16 dargestellt werden. Die Fläche $\bar{w}_{a}^{\prime}+\bar{w}_{b}^{\prime}$ unterscheidet sich von $\bar{w}_{a}^{\prime}-\bar{u}_{b}^{\prime}$ nur durch Drehung um $90^{\circ}$ um das Plattenzentrum.

3) Die dritte Schwingungsfamilie. Wegen der Gleichheit der Schwingungsdauer von $\bar{w}_{a}^{\prime}$ und $\bar{w}_{b}^{\prime}$ kann man die beiden Flächen allgemein so verbinden, dass

$$
\bar{w}=A \bar{w}_{a}^{\prime}+B \bar{w}_{b}^{\prime} \quad \text { oder } \quad \bar{w}^{\prime}=\bar{w}_{a}^{\prime}+\kappa \bar{u}_{b}^{\prime} .
$$

Hierin kann $\kappa$ eine beliebige reelle Zahl sein, und die voran betrachtete erste bzw. zweite Familie dieser Schwingungsart ist nichts anderes als $\bar{w}^{\prime}(49)$ mit dem Sonderwert $\kappa=0$ bzw. $\kappa= \pm 1$.

Die der ersten Wurzel $\mu_{1}=1,59878$ mit $\kappa=-0,2$ sowie der zweiten $\mu_{2}=5,10500$ mit $\kappa=-0,3$ entsprechende Knotenfigur für die dritte Schwingungsfamilie $(0<|\kappa|<1$ ) wird in Abb. 14 mit dem Zeichen (I-3) bzw. (TI-3) dargestellt, während die entsprechenden Schichtinien der $\bar{w}^{\prime}$-Fläche durch Abb. 17 gezeigt werden. 


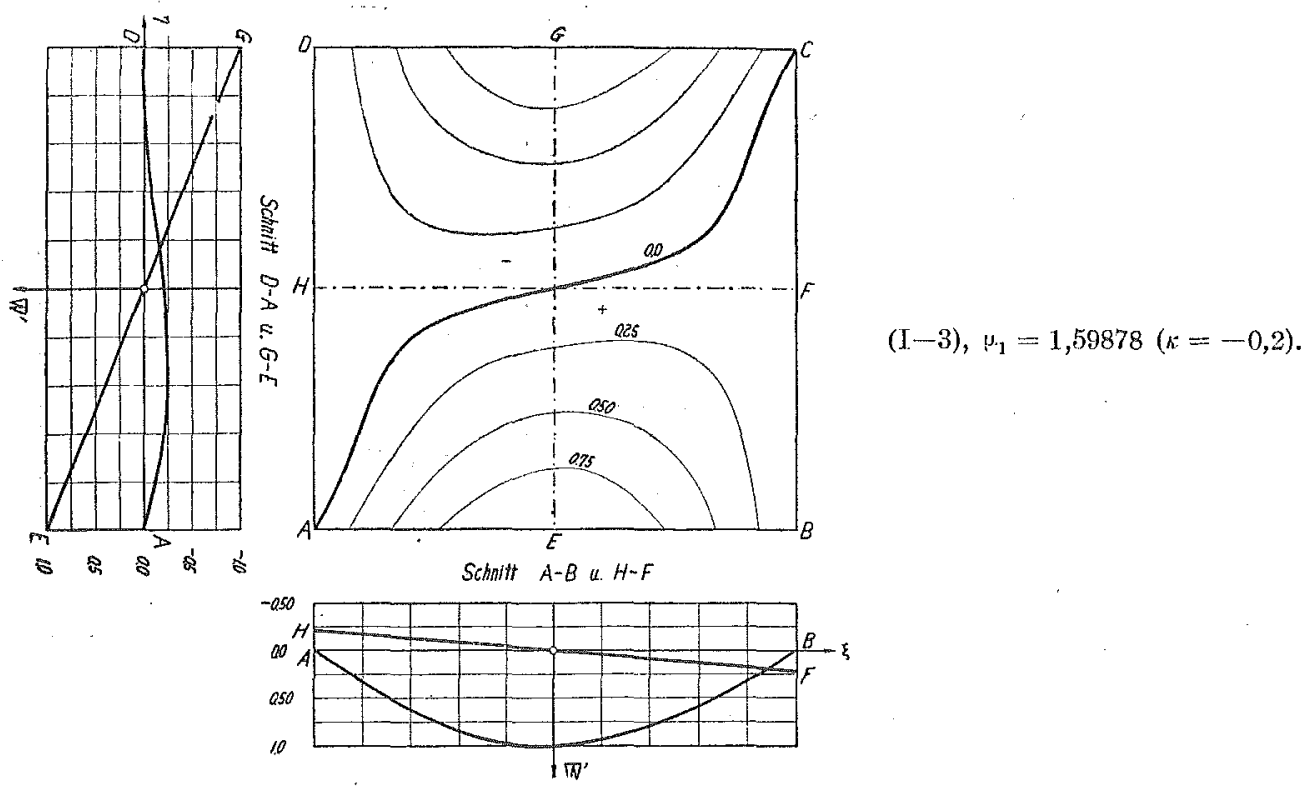

$(I I-3), \varphi_{\cdot g}=5,10500(\kappa=-0,3)$.

Abb. 17. Schichtlinien der Fläche $\bar{u}^{\prime}=\bar{w}_{a^{\prime}}+\kappa \bar{w}_{b}{ }^{\prime}$ fïr die dritte Familie der dritten ,Schwingungsart.
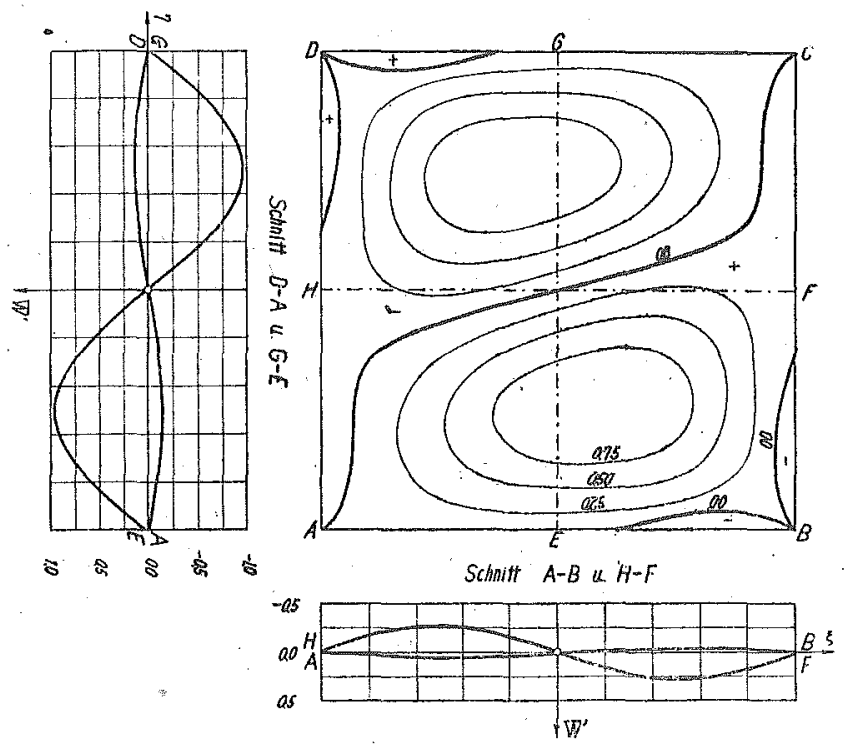

III. Kapitel.

\section{A. Allgemeines.}

11. Die allgemeinen Formeln. Da für die Platte ohne Eckstütze die Ableitung $\frac{\partial^{2} w^{\prime}}{\partial x \partial y}$ in den vier Eckpunkten verschwindet, so gilt hierfür $y^{\prime}(6 a)$ mit Cosinusreihen, d.h. 


$$
\begin{gathered}
w^{\prime}=\sum_{n} X_{n}(\xi) \cos n \pi\left(\frac{1}{2}+\eta\right)+\sum_{m} Y_{m}(\eta) \cos m \pi\left(\frac{1}{2}+\xi\right) \\
(m, n=0,1,2 \ldots)
\end{gathered}
$$

Wegen des Verschwindens der ersten sowie der dritten Ableitung von $\cos m \pi\left(\frac{1}{2}+\xi\right)$ für die Sonderwerte $\xi= \pm 1 / 2$ geht aus den Randbedingungen 3) die Beziehung

$$
\frac{a^{3}}{\pi^{3}} X_{n}^{\prime \prime \prime}\left( \pm \frac{1}{2}\right)-\alpha^{2} n^{2}(2-\nu) \frac{a}{\pi} X_{n}^{\prime}\left( \pm \frac{1}{2}\right)=0
$$

hervor. Mit Rücksicht auf die erste Formel in (eb) ergibt sich

$$
\begin{aligned}
& \pm\left\{A_{n} \lambda_{\alpha n}\left(\lambda_{\alpha n}^{\prime 2}-\nu \alpha^{2} n^{2}\right) \operatorname{Sin} \frac{\pi}{2} \lambda_{\alpha_{n}}+A_{n}^{\prime} \lambda_{\alpha n}^{\prime}\left(\lambda_{\alpha n}^{2}-\nu \alpha^{2} n^{2}\right) \operatorname{Sin} \frac{\pi}{2} \lambda_{\alpha n}^{\prime}\right\} \\
& +A_{n}^{\prime \prime} \lambda_{\alpha_{n}}\left(\lambda_{\alpha_{n}}^{\prime 2}-\nu \alpha^{2} n^{2}\right) \complement_{0} 0 \int_{2}^{\pi} \lambda_{\alpha_{n}}+A_{n}^{\prime \prime \prime} \lambda_{\alpha_{n}}^{\prime}\left(\lambda_{\alpha n}^{2}-\nu \alpha^{2} n^{2}\right)\left(\mathscr{S}_{0} \int \frac{\pi}{2} \lambda_{\alpha_{n}}^{\prime}=0 .\right.
\end{aligned}
$$

Die Sunme bzw. die Differenz der hierdurch dargestellten zwei Gleichungen liefert

$$
A_{n} \lambda_{\alpha_{n}}\left(\lambda_{\alpha_{n}}^{\prime 2}-\nu \alpha^{2} n^{2}\right) \subseteq i n \frac{\pi}{2} \lambda_{\alpha n}+A_{n}^{\prime} \lambda_{\alpha_{n}}^{\prime}\left(\lambda_{\alpha_{n}}^{2}-\nu \alpha^{2} n^{2}\right) \bigodot_{i n} \frac{\pi}{2} \lambda_{\alpha_{n}}^{\prime}=0
$$

bzw.

$$
A_{n}^{\prime \prime} \lambda_{\alpha n}\left(\lambda_{\alpha_{n}}^{\prime 2}-\nu \alpha^{2} n^{2}\right) \operatorname{Cof} \frac{\pi}{2} \lambda_{\alpha n}+A_{n}^{\prime \prime \prime} \lambda_{\alpha_{n}}^{\prime}\left(\lambda_{\alpha_{23}}^{2}-\nu \alpha^{2} n^{2}\right)\left(\mathscr{S}_{0}\right) \frac{\pi}{2} \lambda_{\alpha_{n}}^{\prime}=0
$$

Damit und mit den neu eingeführten Beiwerten $a_{n}$ und $a_{n}^{\prime}$ erhält man

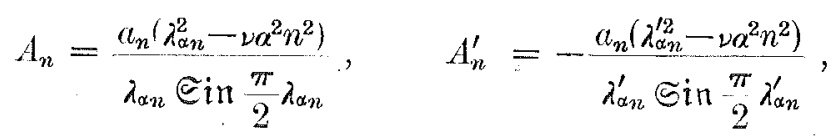

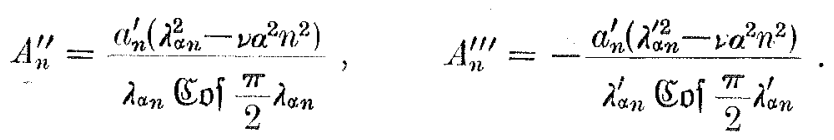

Die Funktion $X_{n}(\xi)$ schreibt sich jetzt, wie folgt:

$$
\begin{aligned}
& X_{n}(\xi)=a_{n} u_{\alpha_{n}}(\xi)+a_{n}^{\prime} v_{\alpha_{n}}(\xi), \quad(n=0,1,2 \ldots) \\
& u_{\alpha_{n}}(\xi)=\left(\lambda_{\alpha_{n}}^{2}-\nu \alpha^{2} n^{2}\right) \frac{\operatorname{So}\left[\pi \lambda_{\alpha_{n}} \xi\right.}{\lambda_{\alpha_{n}} \operatorname{Sin} \frac{\pi}{2} \lambda_{\alpha_{n}}}-\left(\lambda_{\alpha_{n}}^{\prime 2}-\nu \alpha^{2} n^{2}\right) \frac{\operatorname{So}\left[\pi \lambda_{\alpha_{n}}^{\prime} \xi\right.}{\lambda_{\alpha_{n}}^{\prime} \operatorname{Sin} \frac{\pi}{2} \lambda_{\alpha_{n}}^{\prime}},
\end{aligned}
$$

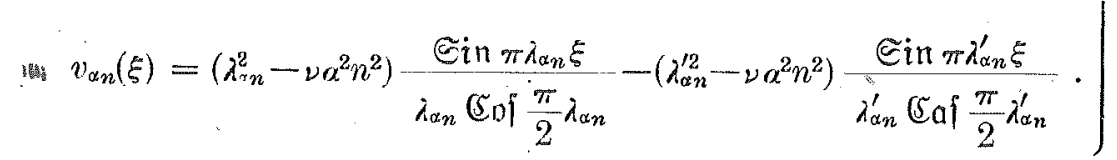


Analog aus den Randbedingungen 4) erhält man

$$
\left.\begin{array}{l}
Y_{m}(\eta)=b_{m} u_{\beta m}(\eta)+b_{m}^{\prime} v_{\beta m}(\eta), \quad(m=0,1,2 \ldots) \\
u_{\beta m}(\eta)=\left(\lambda_{\beta m}^{2}-\nu \beta_{\eta}^{2} m^{2}\right) \frac{\operatorname{Sof} \pi \lambda_{\beta m} \eta}{\lambda_{\beta m} \operatorname{Sin} \frac{\pi}{2} \lambda_{\beta m}}-\left(\lambda_{\beta m}^{\prime 2}-\nu \beta^{2} m m^{2}\right) \frac{\operatorname{Coj} \pi \lambda_{\beta m}^{\prime} \eta}{\lambda_{i m}^{\prime} \operatorname{Sin} \frac{\pi}{2} \lambda_{\beta m}^{\prime}}, \\
v_{\beta m}(\eta)=\left(\lambda_{\beta m}^{2}-\nu \beta^{2} m^{2}\right) \frac{\operatorname{Sin} \pi \lambda_{\beta m} \eta}{\lambda_{\beta m} \operatorname{Coj} \frac{\pi}{2} \lambda_{\beta m}}-\left(\lambda_{\beta m}^{\prime 2}-\nu \beta^{2} m^{2}\right) \frac{\operatorname{Sin} \pi \lambda_{\beta m}^{\prime} \eta}{\lambda_{\beta m}^{\prime} \operatorname{Svj} \frac{\pi}{2} \lambda_{\beta m}^{\prime}}
\end{array}\right\}
$$

Die in (51) und (52) noch verfügbaren unbekannten Beiwerte $a_{n}, a_{n}^{\prime}, b_{m}, b_{m}^{\prime}$ müssen miteinander so verknüpft werden, dass $w^{\prime}(50)$ mit (51) und (52) den übrigon Randbedingungen 1) und 2) genügt.

Mit Rücksicht auf die Beziehung

$$
\begin{aligned}
\cos m \pi\left(\frac{1}{2}+\xi\right) & =\cos \frac{m \pi}{2} \cos m \pi \xi-\sin \frac{m \pi}{2} \sin m \pi \xi \\
& =(-1)^{\frac{m}{2}} \cos m \pi \xi \quad \text { für } m=0,2,4 \ldots, \\
& =(-1)^{\frac{m-1}{2}} \sin m \pi \xi \quad \text { für } m=1,3,5 \ldots
\end{aligned}
$$

merken wir vorläufig an, dass die Zeiger $m$ in (50) bis (52) die geraden bzw. ungeraden Zahlen nehmen, je nachdem die Schwingungsfäche der Platte symmetrisch bzw. antisymmetrisch in bezug auf die Mittellinie $\xi=0$ ist. Analog durchlaufen die Zeiger $n$ die geraden bzw. ungeraden Zahlen, wenn die Schwingungsfläche der Platte sich symmetrisch bzw. antisymmetrisch in bezug auf die Mittellinio $\eta=0$ gestaltet. Zur Betrachtung der Symmetrie- sowie der Antisymmetrieeigenschaft der Schwingungsfläche ist natürlich noch $\mathrm{zu}$ berücksichtigen, dass die beiden Funktionen $X_{n}(\xi)$ und $X_{m}(\eta)$ aus Summen von geraden und ungeraden Funktionen bestehen.

\section{B. Die erste Schwingungsart.}

12. Die allgemeinen Formeln für die rechteckige Platte. Da die Schwingungsfläche hierfür symmetrisch in bezug auf die beiden Mittellinien $\xi=0$ und $\eta=0$ des Rechtecks ist, so ergibt sich sofort aus (50)

$$
\begin{gathered}
w^{\prime}=\sum_{n}(-1)^{\frac{n}{2}} a_{n} u_{\alpha n}(\xi) \cos n_{\pi} \eta+\sum_{m}(-1)^{\frac{m}{2}} b_{m} u_{\beta m}(\eta) \cos m_{\pi \xi} \\
(m, n=0,2,4 \ldots)
\end{gathered}
$$

oder auch

$$
\begin{gathered}
w^{\prime}=a_{0} u_{\alpha 0}(\xi)+b_{0} u_{\beta 0}(\eta) \\
+\sum_{n}(-1)^{\frac{n}{2}} a_{n} u_{\alpha_{n}}(\xi) \cos n \pi \eta+\sum_{m}(-1)^{\frac{m}{2}} b_{m} u_{\beta m}(\eta) \cos m \pi \xi \\
(m, n=2,4,6 \ldots)
\end{gathered}
$$


mit

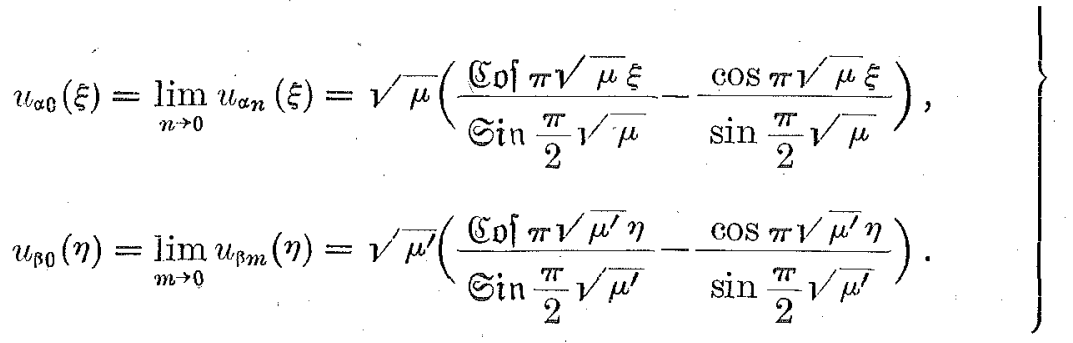

Um weiter die Gleichung für die unbekannten Beiwerte $a_{n}$ und $b_{m}$ zu ermitteln, setzen wir zunächst den Ausdruck für $w^{\prime}(53)$ in die Randbedingungen 1) ein. So lautet

$$
\begin{aligned}
& a_{0} \frac{a^{2}}{\pi^{2}} u_{\alpha 0}^{\prime \prime}\left( \pm \frac{1}{2}\right)+\sum_{n}(-1)^{\frac{n}{2}} a_{n}\left\{\frac{a^{2}}{\pi^{2}} u_{\alpha n_{n}}^{\prime \prime}\left( \pm \frac{1}{2}\right)-\nu \alpha^{2} n^{2} u_{\alpha_{n}}\left( \pm \frac{1}{2}\right)\right\} \cos n \pi^{\eta} \eta \\
& +b_{0} \frac{\nu a^{2}}{\pi^{2}} u_{\beta 0}^{\prime \prime}(\eta)-\sum_{m} b_{m}\left\{m^{2} u_{\beta m}(\eta)-\frac{\nu a^{2}}{\pi^{2}} u_{\beta m}^{\prime \prime}(\eta)\right\}=0 .
\end{aligned}
$$

Darin wird

$$
\begin{aligned}
& \frac{a^{2}}{\pi^{2}} u_{\alpha_{0}}^{\prime \prime}\left( \pm \frac{1}{2}\right)=c \quad \text { (abkü̈rzungsweise) } \\
& =\mu \sqrt{\mu}\left(\left(\mathfrak{g t g} \frac{\pi}{2} \sqrt{\mu}+\operatorname{ctg} \frac{\pi}{2} \sqrt{\mu}\right),\right. \\
& \frac{a^{2}}{\pi^{2}} u_{\alpha_{n}}^{\prime \prime}\left( \pm \frac{1}{2}\right)-\nu \alpha^{2} n^{2} u_{\alpha_{n}}\left( \pm \frac{1}{2}\right)=H_{\alpha_{n}} \quad \text { (abkürrzungsweise) }, \\
& =\frac{\left(\lambda_{\alpha n}^{2}-\nu \alpha^{2} n^{2}\right)^{2}}{\lambda_{\alpha n}} \operatorname{Ctg} \frac{\pi}{2} \lambda_{\alpha_{n}}-\frac{\left(\lambda_{\alpha_{n}}^{\prime 2}-\nu \alpha^{2} \eta_{2}^{2}\right)^{2}}{\lambda_{\alpha_{n}}^{\prime}}\left(\operatorname{ctg} \frac{\pi}{2} \lambda_{\alpha_{n}}^{\prime},\right. \\
& \frac{\nu a^{2}}{\pi^{2}} u_{\beta 0}^{\prime \prime}(\eta)=\nu \alpha^{2} \mu^{\prime} \sqrt{\mu^{\prime}}\left(\frac{\left(\operatorname{\complement n} \pi \sqrt{\mu^{\prime}} \eta\right.}{\operatorname{Cin} \frac{\pi}{2} \sqrt{\mu^{\prime}}}+\frac{\operatorname{Cos} \pi \sqrt{\mu^{\prime}} \eta}{\sin \frac{\pi}{2} \sqrt{\mu^{\prime}}}\right) \\
& m^{2} u_{\iota_{\xi} m}(\eta)-\frac{\nu a^{2}}{\pi^{2}} u_{\beta m}^{\prime \prime}(\eta)=U_{\beta m}(\eta) \quad \text { (abkürzungsweise) } \\
& =\frac{\left(\lambda_{\beta m}^{2}-\nu \beta^{2} m^{2}\right)\left(m^{2}-\nu \alpha^{2} \lambda_{\beta m}^{2}\right)}{\lambda_{\beta m}} \frac{\operatorname{Co}\left[\pi \lambda_{\beta m} \eta\right.}{\operatorname{Sin} \frac{\pi}{2} \lambda_{\beta m}}-\frac{\left(\lambda_{\beta m}^{\prime 2}-\nu \beta^{2} m^{2}\right)\left(m^{2}-\nu \alpha^{2} \lambda_{m m}^{\prime 2}\right)}{\lambda_{\beta m}^{\prime}} \frac{\operatorname{Co} \pi \lambda_{\beta m}^{\prime} \eta}{\operatorname{Sin} \frac{\pi}{2} \lambda_{\beta m}^{\prime}} .
\end{aligned}
$$

Vermöge der Formeln 


$$
\left.\begin{array}{c}
\frac{\operatorname{Sof} \pi \sqrt{\mu^{\prime} \eta}}{\operatorname{Sin} \frac{\pi}{2} \sqrt{\mu^{\prime}}}=\frac{4 \gamma^{\prime} \mu^{\prime}}{\pi}\left\{\frac{1}{2 \mu^{\prime}}+\sum_{n} \frac{(-1)^{\frac{n}{2}}}{n^{2}+\mu^{\prime}} \cos n \pi \eta\right\}, \\
\frac{\cos \pi \sqrt{\mu^{\prime} \eta}}{\sin \frac{\pi}{2} \sqrt{\mu^{\prime}}}=\frac{4 \sqrt{\mu^{\prime}}}{\pi}\left\{\frac{1}{2 \mu^{\prime}}-\sum_{n} \frac{(-1)^{\frac{n}{2}}}{n^{2}-\mu^{\prime}} \cos n \pi \eta\right\}, \\
\frac{\operatorname{Sof} \pi \lambda_{\beta m} \eta}{\operatorname{Sin} \frac{\pi}{2} \lambda_{\beta m}}=\frac{4 \lambda_{\beta m}}{\pi}\left\{\frac{1}{2 \lambda_{\beta m}^{2}}+\sum_{n} \frac{(-1)^{\frac{2}{2}}}{n^{2}+\lambda_{\beta m}^{2}} \cos n \pi \eta\right\}, \\
-\frac{1}{2} \leqq \eta \leqq \frac{1}{2}, \quad(n=2,4,6 \ldots)
\end{array}\right\}
$$

erhält man nach einigen Zwischenrechnungen

$$
\begin{aligned}
& \frac{\nu \alpha^{2}}{\pi^{2}} u_{\beta 0}^{\prime \prime}(\eta)=\frac{8 \nu \alpha^{2} \mu^{\prime}}{\pi}\left\{\frac{1}{2}-\sum_{n}(-1)^{\frac{n}{2}} \frac{\mu^{\prime 2}}{n^{4}-\mu^{\prime 2}} \cos n \pi \eta\right\} \\
& U_{\beta m}(\eta)=\frac{8 \mu^{\prime}}{\pi}\left\{\frac{\nu \alpha^{2} \mu^{\prime 2}}{2\left(\beta^{4} m^{4}-\mu^{\prime 2}\right)}+\sum_{n}(-1)^{\frac{n}{2}} \frac{(1-\nu)^{2} m^{2} n^{2}+\nu \alpha^{2} \mu^{\prime 2}}{\left(n^{2}+\lambda_{i m}^{2}\right)\left(n^{2}+\lambda_{i m}^{\prime 2}\right)} \cos n \pi \eta\right\} .
\end{aligned}
$$

Das Einsetzen von $(b),(c),(d)$ und $(e)$ in $(a)$ liefert

$$
\begin{aligned}
a_{0} c & +\frac{4 \nu \alpha^{2} \mu^{\prime}}{\pi}\left(b_{0}-\sum_{r} b_{r} \frac{\mu^{\prime 2}}{\beta^{4} r^{4}-\mu^{\prime 2}}\right) \\
& +\sum_{n}(-1)^{\frac{n}{2}}\left\{a_{n} H_{\alpha_{n}}-\frac{\delta \mu^{\prime}}{\pi}\left[b_{0} \frac{\nu \alpha^{2} \mu^{\prime 2}}{n^{4}-\mu^{\prime 2}}+\sum_{r} b_{r} \frac{(1-\nu)^{2} \eta^{2} n^{2}+\nu \alpha^{2} \mu^{\prime 2}}{\left(n^{2}+\lambda_{\beta r}^{2}\right)\left(n^{2}+\lambda_{\beta r}^{2}\right)}\right]\right\} \cos n \pi \eta=0 .
\end{aligned}
$$

Da diese Gleichung für jeden Wert von $\eta$ erfüllt sein soll, so muss die Summe der konstanten Glieder und der mit $\cos n \pi \eta$ behaftete Ausdruck teilweise verschwinden. Also erhält man

mit

$$
\begin{gathered}
a_{0}+\frac{4 \nu \alpha^{2} \mu^{\prime}}{\pi c}\left(b_{0}-\sum_{r} b_{r} \frac{\mu^{\prime 2}}{\beta^{4} \gamma^{4}-\mu^{\prime 2}}\right)=0 \\
a_{n}-\frac{8 \mu^{\prime}}{\pi H_{\alpha, n}}\left\{b_{0} \frac{\nu \alpha^{2} \mu^{\prime 2}}{n^{4}-\mu^{2}}+\sum_{r} b_{r} \frac{(1-\nu)^{2} \eta^{2} n^{2}+\nu \alpha^{2} \mu^{\prime 2}}{\left(n^{2}+\lambda_{\beta r}^{2}\right)\left(n^{2}+\lambda_{\beta r}^{2}\right)}\right\}=0 \\
(n, r=2,4,6 \ldots)
\end{gathered}
$$

$$
\begin{aligned}
H_{\alpha n} & \equiv \frac{\left(\lambda_{\alpha n}^{2}-\nu \alpha^{2} n^{2}\right)^{2}}{\lambda_{\alpha_{n}}}\left(\zeta \operatorname{tg} \frac{\pi}{2} \lambda_{\alpha n}-\frac{\left(\lambda_{\alpha_{n}}^{2}-\nu \alpha^{2} n^{2}\right)^{2}}{\lambda_{\alpha_{n}}^{\prime}} \Subset \operatorname{tg} \frac{\pi}{2} \lambda_{\alpha n}^{\prime}\right. \\
c & =\lim _{n \rightarrow 0} H_{\alpha_{n}} \equiv \mu \sqrt{\mu}\left(\circlearrowleft \operatorname{tg} \frac{\pi}{2} \sqrt{\mu}+\operatorname{ctg} \frac{\pi}{2} \sqrt{\mu}\right)
\end{aligned}
$$


Analog ergibt sich aus den Randbedingungen 2)

$$
\begin{gathered}
b_{0}+\frac{4 \nu \beta^{2} \mu}{\pi c^{\prime}}\left(a_{0}-\sum_{s} a_{s} \frac{\mu^{2}}{\alpha^{4} s^{4}-\mu^{2}}\right)=0, \\
b_{m}-\frac{8 \mu}{\pi H_{\beta m}}\left\{a_{0} \frac{\nu \beta^{2} \mu^{2}}{m^{4}-\mu^{2}}+\sum_{s} a_{s} \frac{(1-\nu)^{2} m^{2} s^{2}+\nu \beta^{2} \mu^{2}}{\left(m^{2}+\lambda_{\alpha s}^{2}\right)\left(m^{2}+\lambda_{\alpha s}^{\prime 2}\right)}\right\}=0 \\
(m, s=2,4,6 \ldots)
\end{gathered}
$$

mit

$$
\begin{aligned}
H_{\beta m} & \equiv \frac{\left(\lambda_{\beta m}^{2}-\nu \beta^{2} m^{2}\right)^{2}}{\lambda_{\beta m}} \operatorname{ctg} \frac{\pi}{2} \lambda_{\beta m}-\frac{\left(\lambda_{\beta m}^{\prime 2}-\nu \beta^{2} m^{2}\right)^{2}}{\lambda_{\beta m}^{\prime}}\left(\operatorname{tg} \frac{\pi}{2} \lambda_{\beta m}^{\prime},\right. \\
c^{\prime} & =\lim _{m \rightarrow 0} H_{\beta m} \equiv \mu^{\prime} v^{\prime} \overline{\mu^{\prime}}\left(\left(5 \operatorname{tg} \frac{\pi}{2} \sqrt{\mu^{\prime}}+\operatorname{ctg} \frac{\pi}{2} v^{\prime} \overline{\mu^{\prime}}\right)\right.
\end{aligned}
$$

Aus der ersten Gleichung in (56) und der ersten in (57) erhält man

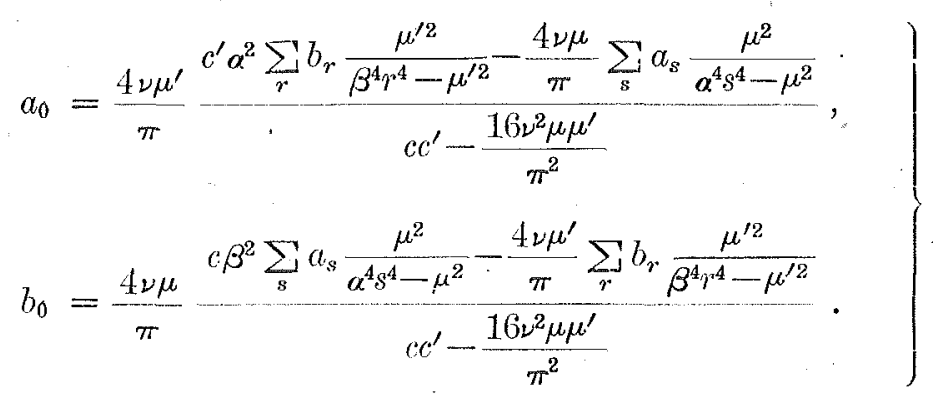

Durch geeignete weitere Rechnungen kann man ans (56) bis (58) die Frequenzgleichung für diesen Schwingungsfall ableiten. Da aber die diesbezüg-. lichen allgemeinen Rechnungen für die Rechteckplatte $(a \neq b)$ ziemlich mühsam sind, so werden sie bei der Untersuchung der quadratischen Platte in den. kommenden Ziff. angegeben.

13. Die Formeln und die numerischen Rechenergebnisse für die quadratische Platte. Da für die quadratische Platte $m=n=2,4,6 \cdots, \alpha=\beta=1$, $\mu^{\prime}=\mu$ ist, so kann man wie früher in allen Formeln hierfür einfachheitshalber $\lambda_{n}$ für $\lambda_{\alpha_{n}}$ und $\lambda_{\beta m}$, $\lambda_{n}^{\prime}$ für $\lambda_{\alpha_{n}}^{\prime}$ und $\lambda_{\beta m}^{\prime}$, $u_{0}$ für $u_{\alpha_{0}}$ und $u_{\beta 0}, u_{n}$ für $u_{\alpha_{n}}$ und $u_{\beta m}$ schreiben.

1) Die erste Schwingungsfamilie. Da die Schwingungsfläche sich bei diesem Falle symmetrisch in bezug nicht nur auf die beiden Mittellinien, sondern auch auf die beiden Diagolanen des Quadrats'gestaltet, so ergibt sich

$$
a_{0}=b_{0}, \quad a_{n}=b_{n},
$$

Damit geht sofort aus (56) 
mit

$$
\begin{aligned}
& a_{0}=\frac{2 \nu}{2 \nu+\frac{\pi c}{2 \mu}} \sum_{s} a_{s} \frac{\mu^{2}}{s^{4}-\mu^{2}}, \\
& a_{n}-\frac{8 \mu}{\pi \Pi_{n}}\left\{a_{0} \frac{\nu \mu^{2}}{n^{4}-\mu^{2}}+\sum_{s} a_{s} \frac{(1-\nu)^{2} n^{2} s^{2}+\nu \mu^{2}}{\left(n^{2}+s^{2}\right)^{2}-\mu^{2}}\right\}=0 \\
& \quad(n, s=2,4,6 \ldots)
\end{aligned}
$$

$$
H_{n} \equiv \frac{\left(n^{2}-\nu n^{2}+\mu\right)^{2}}{\sqrt{n^{2}+\mu}}\left(\operatorname{tg} \frac{\pi}{2} \sqrt{n^{2}+\mu}-\frac{\left(n^{2}-\nu n^{2}-\dot{\mu}\right)^{2}}{\sqrt{n^{2}-\mu}}\left(\operatorname{stg} \frac{\pi}{2} \sqrt{n^{2}-\mu}\right)\right.
$$

hervor. Durch Einführung des durch die erste Gleichung (59) dargestellten Ausdruck für $a_{0}$ in die zweite erhält man

$$
a_{n}+\sum_{s} K_{n s} a_{s}=0, \quad(n, s=2,4,6 \ldots)
$$

mit

$$
\left.K_{n s}=-\frac{8 \mu}{\pi H_{n}}\left\{\frac{2 \nu^{2} \mu^{4}}{\left(2 \nu+\frac{\pi c}{2 \mu}\right)\left(n^{4}-\mu^{2}\right)\left(s^{4}-\mu^{2}\right)}+\frac{(1-\nu)^{2} n^{2} s^{2}+\nu \mu^{2}}{\left(n^{2}+s^{2}\right)^{2}-\mu^{2}}\right\} \cdot\right\}
$$

Hierin ist der Ausdruck für e derselbe wie der in (56).

Die Elimination der unbekannten Beiwerte $a_{n}\left(=a_{2}, a_{4}, a_{6}, \ldots\right)$ aus dem aus (60) hervorgehenden System von unendlich vielen homogen linearen Gleichungen für $a_{n}$ liefert die Frequenzgleichung

$$
\Delta=\left|\begin{array}{rrrrr}
1+K_{22} & K_{24} & K_{26} & K_{28} & \ldots \\
K_{42} & 1+K_{44} & K_{46} & K_{48} & \ldots \\
K_{62} & K_{64} & 1+K_{66} & K_{68} & \ldots \\
K_{22} & K_{84} & K_{86} & 1+K_{88} & \ldots \\
\ldots & \ldots & \ldots & \ldots & \ldots
\end{array}\right|=0 .
$$

\begin{tabular}{|c|c|c|c|c|c|c|c|}
\hline$\mu$ & $n$ & $\alpha_{n}$ & $2 \cdot n$ & $\lambda^{2} n-v n^{2}$ & $x_{n}^{\prime}$ & $x^{\prime 2} n-n^{2}$ & $\begin{array}{c}\text { Abb. } 18 . \\
\text { Knotenlinien. }\end{array}$ \\
\hline \multirow{5}{*}{ 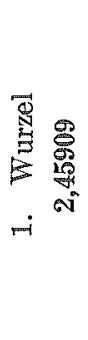 } & 0 & 8,519346 & - & - & - & - & \\
\hline & 2 & 1,000000 & 2,541474 & 5,25909 & 1,241334 & 0,34091 & \\
\hline & 4 & 0,042253 & 4,296405 & 13,65909 & 3,679898 & 8,74091 & \\
\hline & 6 & 0,011729 & 6,201540 & 27,65909 & 5,791450 & 22,74091 & \\
\hline & 8 & 0,004940 & 8,152246 & 47,25909 & 7,844800 & 42,34091 & (I) \\
\hline
\end{tabular}

Die aus $\lrcorner_{4}=0$ ermittelten Werte der ersten fünf Wurzeln $\mu$ für die quadratische Platte mit $\nu=0,3$ sind in Tabelle 7 zusammengestellt.

Tabelle 7. Die Werte von $\mu$ und die der verschiedenen Beiwerte für die erste Familie d'er ersten Schwingungsart der quadratischen Platte mit $\nu=0,3$. 
Die Eigenschwingungen und Klangfiguren der vierseitig freien rechteckigen Platte.

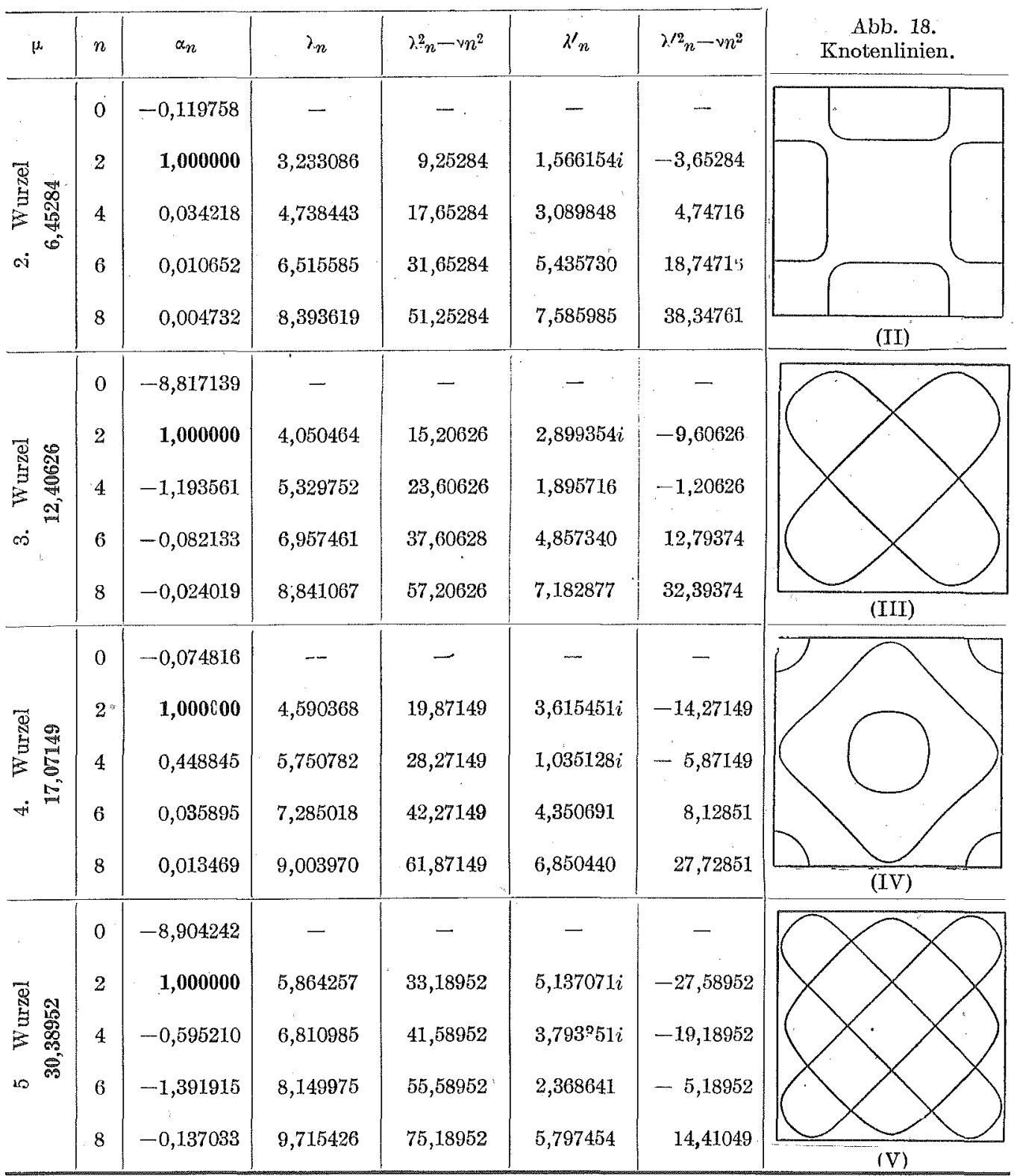




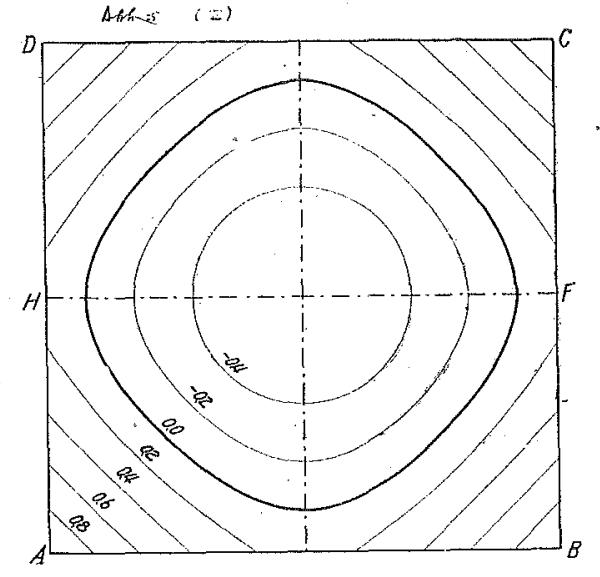

Schnift $A-B$ u. H-F

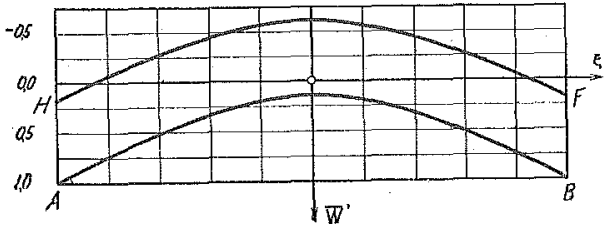

(I), $\mu_{1}=2,45909$.

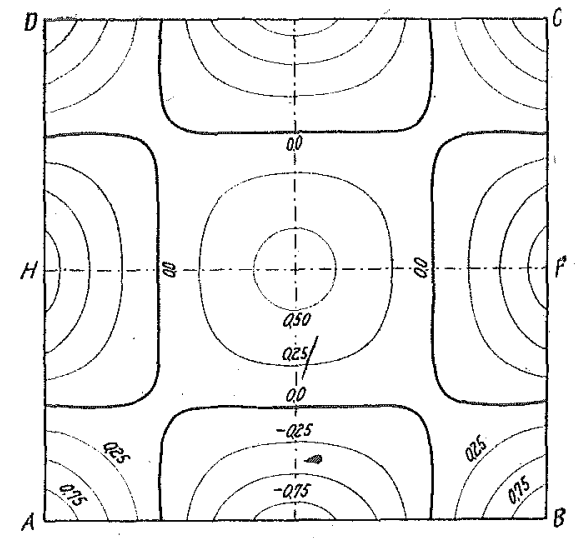

Schiaitf $A-B$ u. H-F

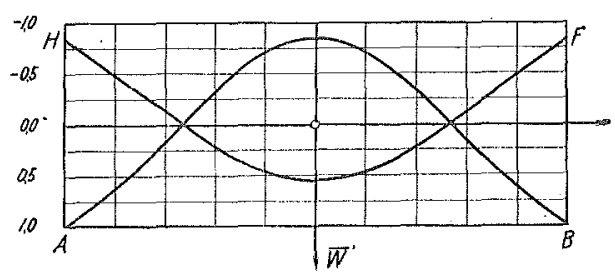

(II), $\mu_{\mathrm{O}}=6,45284$.
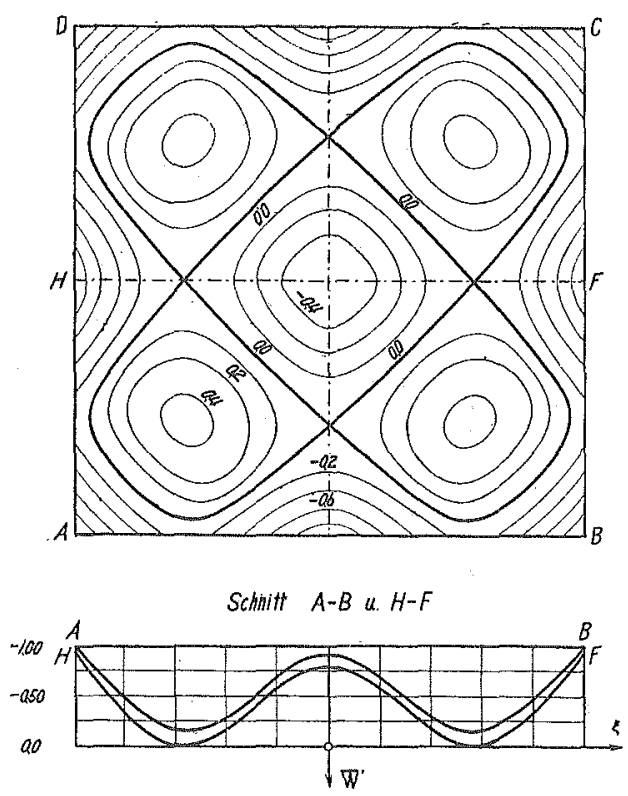

(III), $\mu_{3}=12,40626$. 


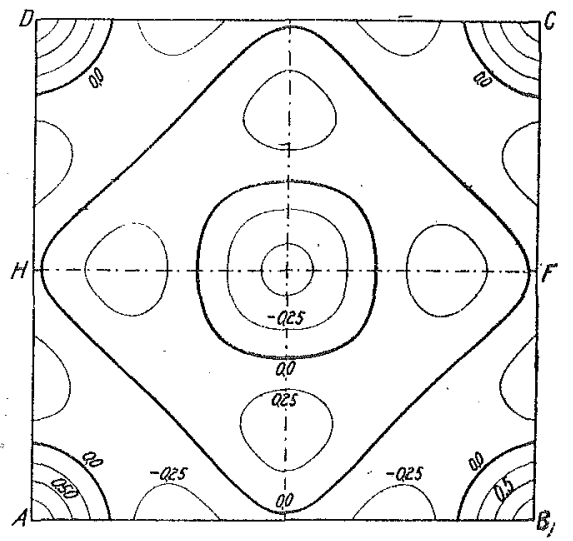

Schnitt. $A-B$ u. $H-F$,

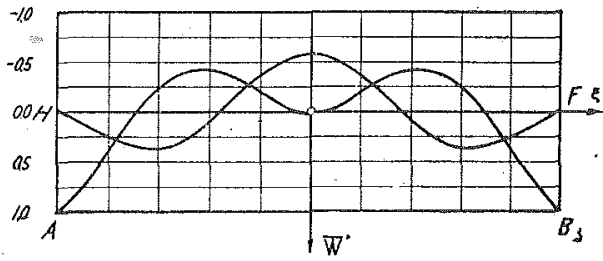

(IV), $\mu_{4}=17,07149$

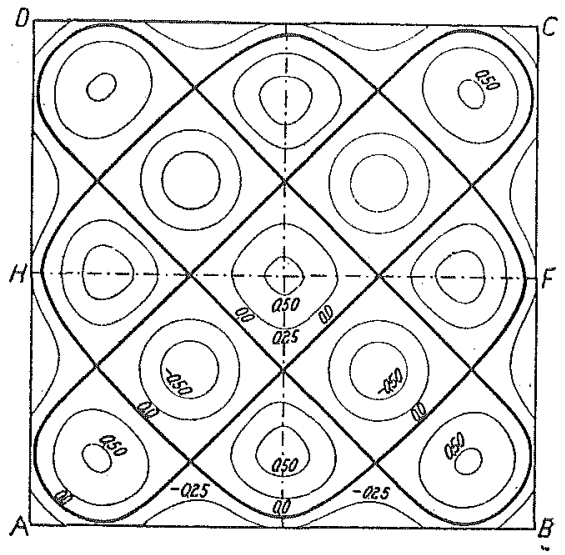

Schnitt $A-B$ \& $H-F$

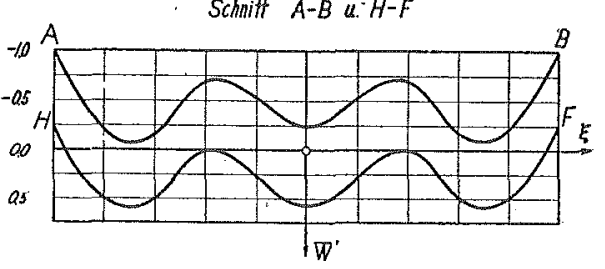

(V), $\mu_{5}=30,38952$.

Abb. 19. Schichtlinien der $\bar{w}^{\prime}-$ Fläche für die erste Familie der ersten Schwingungsart.

Weiter schreibt sich die Schwingungsfläche aus (54), wie folgt:

$$
\begin{aligned}
& w^{\prime}=a_{2} \bar{w}^{\prime}, \\
& \bar{w}^{\prime}=a_{0}\left\{u_{0}(\xi)+u_{0}(\eta)\right\} \\
& +\sum_{n}(-1)^{\frac{n}{2}} \alpha_{n}\left\{u_{n}(\xi) \cos n \pi \eta+u_{n}(\eta) \cos n \pi \xi\right\}, \\
& (n=2,4,6 \ldots) . \\
& \quad \alpha_{0}=\frac{a_{0}}{a_{2}}, \quad \alpha_{n}=\frac{a_{n}}{a_{2}}, \quad\left(\alpha_{2}=1\right) .
\end{aligned}
$$

Hierin können die Beiwerte $a_{n}$ durch die Auflösung der aus

$$
\alpha_{n}+\sum_{s} K_{n s} \alpha_{s}=0, \quad\left(\alpha_{2}=1\right) \quad(n=2,4,6,8)
$$

hervorgehenden linearen Gleichungen für $\alpha_{4}, \alpha_{6}, \alpha_{8}$ ermittelt werden, während der Wert von $\alpha_{0}$ aus

$$
\alpha_{0}=\frac{2 \nu}{2 \nu+\frac{\pi c}{2 \mu}} \sum_{s} \alpha_{s} \frac{\mu^{2}}{s^{4}-\mu^{2}}
$$


ausgerechnet wird. Die den ersten fünf Wurzeln angehörenden Knotenlinien $\bar{w}^{\prime}=0$ werden schematisch in Abb. 18 dargestellt. Die in Abb: 19 gezeigten Figuren sind die entsprechenden Schichtlinien der $\bar{u}^{\prime}$-Fläche.

2) Die zweite Schwingungsfamilie. Hierin wollen wir uns mit der zweiten Familie der ersten Schwingungsart beschäftigen, in der die Platte symmetrisch in bezug auf die beiden Mittellinien, antisymmetrisch dagegen in bezug auf die beiden Diagonalen schwingt. Die Schwingungsfäche hat hierbei immer mindestens zwei Diagonalen als Knotenlinien und wird wegen $\cdot b_{n}\left(=b_{m}\right)=-a_{n}$ durch

$$
\begin{gathered}
\bar{w}^{\prime}=a_{n}\left\{u_{0}(\xi)-u_{0}(\eta)\right\}+\sum_{n}(-1)^{\frac{n}{2}} \alpha_{n}\left\{u_{n}(\xi) \cos n_{\pi} \eta-u_{n}(\eta) \cos n \pi \xi\right\} \\
(n=2,4,6 \ldots)
\end{gathered}
$$

ausgedrïckt. Die entsprechende Frequenzgleichung ist dieselbe wie (61) mit Umkehrung des Vorzeichens von $K_{n \boldsymbol{n}}(60)$. Die Wurzeln, die aus der Lösung der durch diese Änderung hergestellten Frequenzgleichung gefunden werden, sind natürlich verschieden von den für die letzten Schwingungsfamilie. Die Rechenergebnisse für die ersten fünf Wurzeln sind in Tabelle 8 zusammengestellt, während die entsprechenden Knotenfiguren durch $\mathrm{Abb} .20$ und die Schichtlinien der $\bar{w}^{\prime}$-Fläche durch $\mathrm{Abb} .21$ gezeigt werden.

T'abelle 8. Die Werte von $\mu$ und die der verschiedenen Beiwerte für die zweite Familie der ersten Schwingungsart der

\begin{tabular}{|c|c|c|c|c|c|c|c|}
\hline$\mu$ & $n$ & $\alpha_{n}$ & $\lambda_{n}$ & $7^{2} n^{--v n^{2}}$ & $x_{n}^{\prime}$ & $2 n^{4} n^{2}-v n^{2}$ & $\begin{array}{c}\text { Abb. } 20 . \\
\text { Knotenlinien. }\end{array}$ \\
\hline \multirow{5}{*}{ 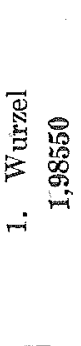 } & 0 & $-19,460604$ & - & - & - & - & \multirow[b]{5}{*}{ (I) } \\
\hline & 2 & 1,000000 & 2,446529 & 4,78550 & 1,419329 & 0,81450 & \\
\hline & 4 & 0,002636 & 4,240932 & 13,18550 & 3,743594 & 9,21450 & \\
\hline & 6 & $-0,004871$ & 6,163238 & 27,18550 & 5,832194 & 23,21450 & \\
\hline & 8 & $-0,00.904$ & 8,123145 & 46,78550 & 7,874926 & 42,81450 & \\
\hline \multirow{5}{*}{ 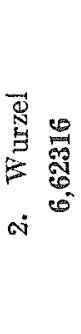 } & 0 & 3,936979 & - & - & - & - & \multirow[b]{5}{*}{ (II) } \\
\hline & 2 & 1,000000 & 3,259320 & 9,42316 & $1,619617 i$ & $-3,82316$ & \\
\hline & 4 & $-0,098351$ & 4,756381 & 17,82316 & 3,062163 & 4,57684 & \\
\hline & 6 & $-0,015073$ & 6,528640 & 31,82316 & 5,420041 & 18,57684 & \\
\hline & 8 & $-0,004513$ & 8,403759 & 51,42316 & 7,574751 & 38,17684 & \\
\hline
\end{tabular}
quadratischen Platte mit $\nu=0,3$. 
Die Eigenschwingungen und Klangfiguren der vierseitig freien rechteckigen Platte.

\begin{tabular}{|c|c|c|c|c|c|c|c|}
\hline$\mu$ & $n$ & $\alpha_{n}$ & $\lambda_{n}$ & $\lambda_{n}^{2}-v n^{2}$ & $2 \cdot n^{\prime}$ & $2 / 2 n^{2}-v n^{2}$ & $\begin{array}{c}\text { Abb. } 20 . \\
\text { Knotenlinien. }\end{array}$ \\
\hline \multirow{5}{*}{ 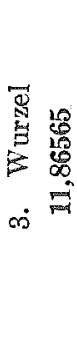 } & 0 & 3,848262 & - & - & - & - & \\
\hline & 2 & 1,000000 & 3,983171 & 14,66565 & $2,804577 i$ & $\neg 9,06565$ & \\
\hline & 4 & $-0,480908$ & 5,278792 & 23,06565 & 2,033310 & -066565 & \\
\hline & 6 & $-0,028448$ & 6,918502 & 37,06565 & $4,9126^{\circ} 71$ & 13,33435 & \\
\hline & 8 & $-0,004534$ & 8,710088 & 56,66565 & 7,220411 & 32,93435 & \\
\hline \multirow{5}{*}{ 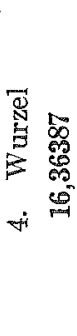 } & 0 & $-0,028327$ & - & - & - & - & \\
\hline & 2 & 1,000000 & 4,512635 & 19,16387 & $3,516230 i$ & $-13,56387$ & \\
\hline & 4 & $-0,244277$ & 5,688925 & 27,56387 & $0,603217 i$ & $-5,16387$ & \\
\hline & 6 & $-0,013635$ & 7,236287 & 41,56387 & 4,431266 & 8,83613 & \\
\hline & 8 & $-0,002967$ & 8,964590 & 61,16387 & 6,901892 & 28,43613 & \\
\hline \multirow{5}{*}{ 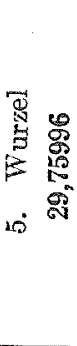 } & 0 & 5,793543 & - & - & - & _- & \\
\hline & 2 & 1,000000 & 5,810332 & 32,55996 & $5,075427 i$ & $-26,95996$ & \\
\hline & 4 & 0,663307 & 6,764612 & 40,95996 & $3,709441 i$ & $-18,55996$ & \\
\hline & 6 & $-0,616989$ & 8,109251 & 54,95996 & 2,498008 & - 4,55996 & \\
\hline & 8 & $-0,057321$ & 9,682972 & 74,55996 & 5,851499 & 15,04004 & \\
\hline
\end{tabular}




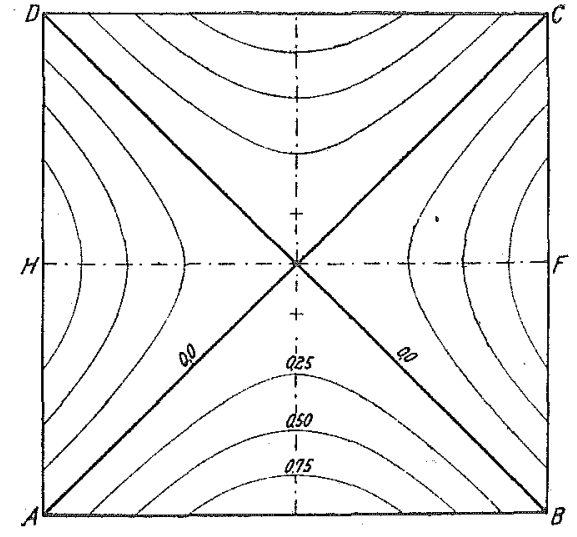

Schnitt $A-B$ u. H-F

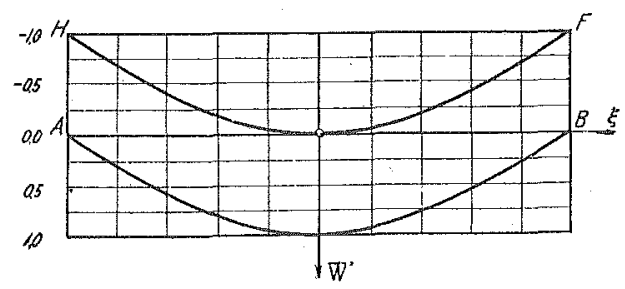

(I), $\mu_{1}=1,98550$.

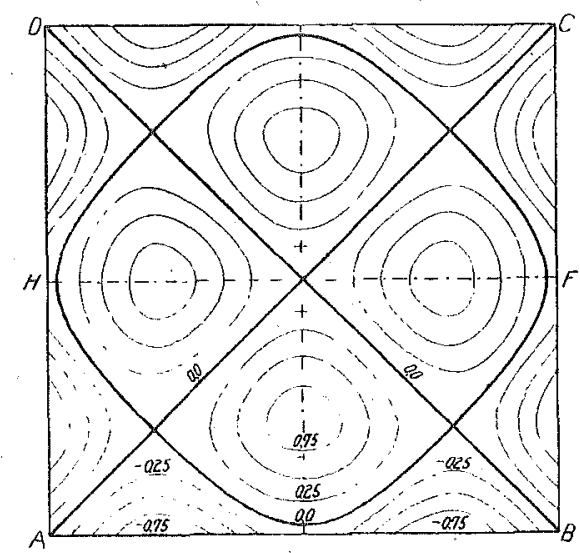

Schnitf $A-B \cup H-F$

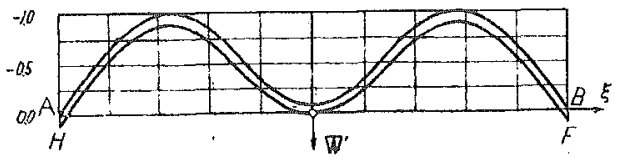

(III), $\mu_{3}=11,86565$.

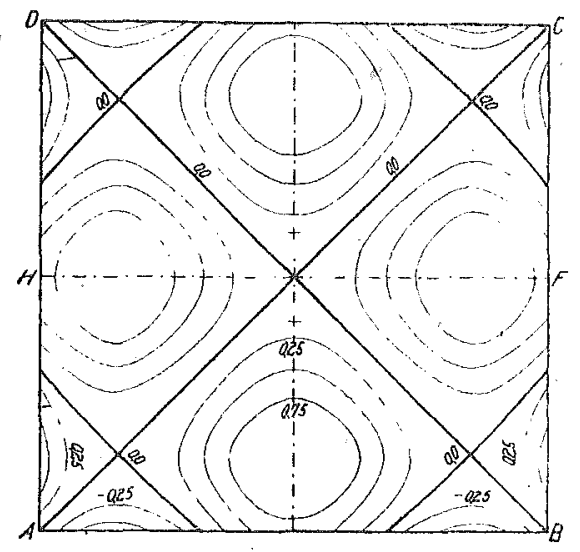

Schmitt A-B u. H-F

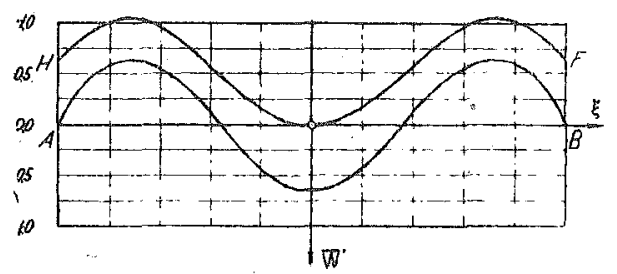

(II) $, \mu_{\text {. }}=6,62316$.
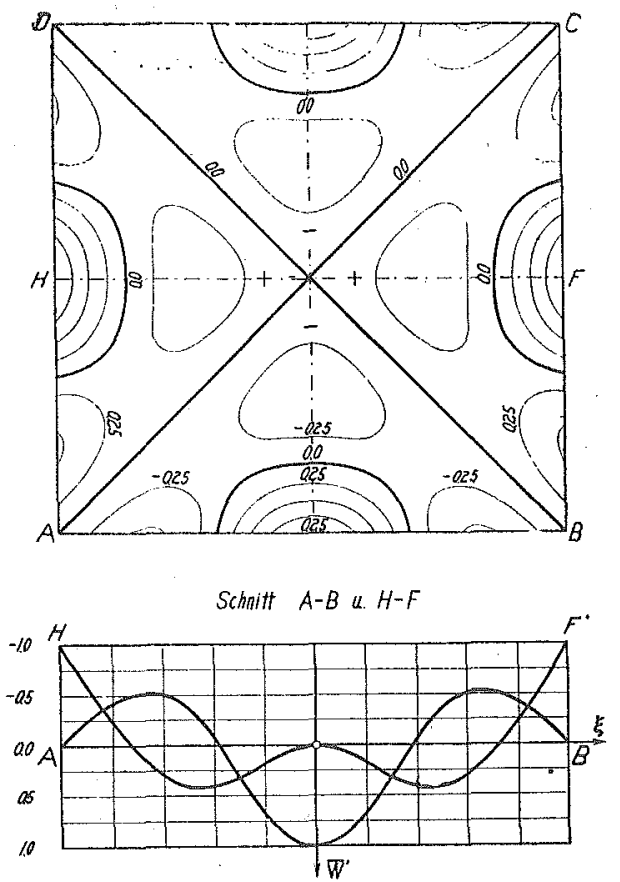

(IV), $\mu_{d}=16,36387$. 


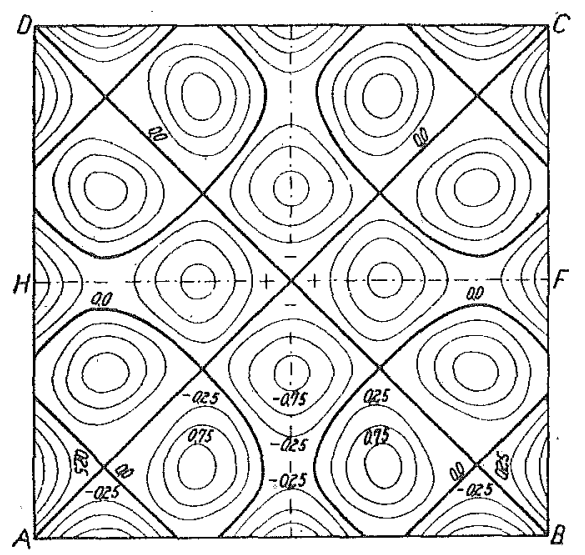

Schnitf $A-B \quad u \cdot H-F$

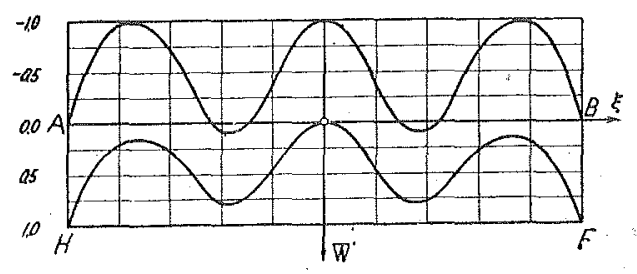

(V), $\mu_{5}=29,75996$.

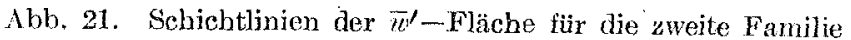
der ersten Schwingungsart.

14. Die Näherungsformeln für die quadratische Platte. Wenn man in (62) bzw. (63) alle Glieder der unendlichen Reihen vernachlässigt, so erhält man lediglich

$$
\begin{aligned}
& w^{\prime}=a_{0}\left\{u_{0}(\xi)+u_{0}(\eta)\right\}, \\
& =a_{0} \sqrt{\mu}\left(\frac{\operatorname{Sof} \pi V \xi}{\operatorname{Sin} \frac{\pi}{2} \sqrt{\mu}}-\frac{\cos \pi V \xi \xi}{\sin \frac{\pi}{2} \sqrt{\mu}}+\frac{(\operatorname{Sol} \pi / \mu \eta}{\operatorname{Sin} \frac{\pi}{2} \sqrt{\mu}}-\frac{\cos \pi V \mu \eta}{\sin \frac{\pi}{2} V \mu}\right), \\
& w^{\prime}=a_{0}\left\{u_{0}(\xi)-u_{0}(\eta)\right\},
\end{aligned}
$$

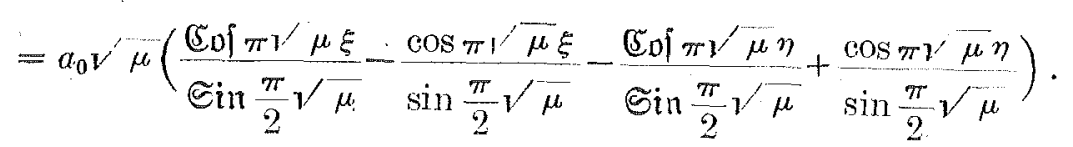

Diose sind die Rayleighschen Näherungsformeln 1), und die Gleichung (64a) bzw. (64b) gehört zu der ersten bzw. zweiten Familie der ersten Schwingungsart der

1) Lord Rayleigh, "Theory of Sound," 1, S. 372, 1929. 
quadratischen Platte. Die beiden Gleichungen genügen von vornherein den Randbedingungen

$$
\left(\frac{\partial^{3} w^{\prime}}{\partial x^{3}}\right)_{\xi= \pm \frac{1}{2}}=0 \quad \text { und } \quad\left(\frac{\partial^{3} w^{\prime}}{\partial y^{3}}\right)_{\eta= \pm \frac{1}{2}}=0 .
$$

Die Werte von $\mu$ werden durch die aus den anderen Randbedingungen

$$
\left(\frac{\partial^{2} w^{\prime}}{\partial x^{2}}\right)_{\xi- \pm \frac{1}{2}}=0 \quad \text { oder } \quad\left(\frac{\partial^{2} w^{\prime}}{\partial y^{2}}\right)_{\eta= \pm \frac{1}{2}}=0
$$

hervorgehenden Gleichung, nämlich durch

$$
\mathfrak{S t g} \frac{\pi}{2} \sqrt{\mu}+\operatorname{ctg} \frac{\pi}{2} \sqrt{\mu}=0 \quad \text { oder } \quad \mathfrak{I} \mathfrak{g} \frac{\pi}{2} \sqrt{\mu}+\operatorname{tg} \frac{\pi}{2} \sqrt{\mu}=0
$$

bestimmt. Dies ist nichts anderes als die Frequenzgleichung für die symmetrischen Schwingungen des geraden Stabes mit freien Enden. Es ist bemerkenswert, dass die Gleichung (6.5) auch aus $\lim _{n \rightarrow 0} H_{n}=c=0$ hervorgeht.

Die Auflösung von (65) nach $\mu$ liefert

$$
\begin{array}{llll}
\text { 1. Wurzel } & \text { 2. Wurzel } & \text { 3. Wurzel } & \text { 4. Wurzel } \\
\mu=2,26689, & 12,25007, & 30,25000, & 56,25000 .
\end{array}
$$

Diese Werte von $\mu$ gelten sowohl für die erste als auch für die zweite Familie, weil eine und dieselbe Frequenzgleichung (65) aus (64a) wie auch aus (64b) hervorgeht. Theoretisch ist das aber nicht der Fall.

Man erkennt, dass die oben angeschriebene erste, zweițe bzw. dritte Wurzel der in Tabelle 7 oder 8 stehenden ersten, dritten bzw. fünften Wurzel mit nur wenigem Fehler entspricht. Ferner sieht man, dass die den aus den theoretischen Formeln hervorgehenden zweiten, vierten u.s.w. Wurzeln entsprechenden Wurzeln nicht aus der Näherungsformel (65) folgen.

\section{Die zweite Schwingungsart.}

15. Die allgemeinen Formeln für die rechteckige Platte. Nunmehr denken wir uns den Fall, in dem die Schwingungsfläche der Platte antisymmetriseh in bezug auf die beiden Mittellinien $\xi=0$ und $\eta=0$ des Rechtecks ist. Die Schwingungsfläche hat hierbei mindestens zwei Mittellinien als ihre Knotenlinien und wird durch

$$
\begin{gathered}
w^{\prime}=\sum_{n}(-1)^{\frac{n-1}{2}} a_{n}^{\prime} v_{\alpha_{n}}(\xi) \sin n \pi \eta+\sum_{m}(-1)^{\frac{m-1}{2}} b_{m}^{\prime} v_{\beta m}(\eta) \sin m_{\pi} \xi \\
(m, n,=1,3,5 \ldots)
\end{gathered}
$$


ausgedrückt. Die darin enthaltenden Beiwerte $a_{n}^{\prime}$ und $b_{m}^{\prime}$ werden wegen der Randbedingungen 1) miteinander so verknüpft, dass

$$
\begin{aligned}
\sum_{n}(-1)^{\frac{n-1}{2}} a_{n}^{\prime}\left\{\frac{a^{2}}{\pi^{2}} v_{\alpha n}^{\prime \prime}\left( \pm \frac{1}{2}\right)-\nu \alpha^{2} n^{2} v_{\alpha_{n}}\left( \pm \frac{1}{2}\right)\right\} \sin n \pi \eta \\
\\
-\sum_{m} b_{m}^{\prime}\left\{m^{2} v_{\beta m}(\eta)-\frac{\nu a^{2}}{\pi^{2}} v_{\beta m}^{\prime \prime}(\eta)\right\}=0
\end{aligned}
$$

Mit Rücksicht auf die Beziehung

$$
\frac{a^{2}}{\pi^{2}} v_{\alpha n}^{\prime \prime}\left( \pm \frac{1}{2}\right)-\nu \alpha^{2} n^{2} v_{\alpha_{n}}\left( \pm \frac{1}{2}\right)=\frac{\left(\lambda_{\alpha n}^{2}-\nu \alpha^{2} n^{2}\right)^{2}}{\lambda_{\alpha n}} \mathfrak{I} \mathfrak{g} \frac{\pi}{2} \lambda_{\alpha n}-\frac{\left(\lambda_{\alpha n}^{2}-2 \alpha^{2} n^{2}\right)^{2}}{\lambda_{\alpha n}^{\prime}} \mathfrak{I} \mathfrak{g} \frac{\pi}{2} \lambda_{\alpha_{n}}^{\prime}
$$

und vermöge der Formel

$$
\begin{aligned}
\frac{\operatorname{Sin} \pi \lambda_{\beta m} \eta}{\operatorname{Cof} \frac{\pi}{2} \lambda_{\beta m}}= & \frac{4 \lambda_{\beta m}}{\pi} \sum_{n} \frac{(-1)^{\frac{n-1}{2}}}{n^{2}+\lambda_{B m}^{2}} \sin n \pi \eta, \\
& -\frac{1}{2} \leqq \eta \leqq \frac{1}{2}, \quad(n=1,3,6 \ldots)
\end{aligned}
$$

ergibt sich aus (a)

mit

$$
\begin{array}{r}
a_{n}^{\prime}-\frac{8 \mu^{\prime}}{\pi G_{\alpha n}} \sum_{r} b_{r}^{\prime} \frac{(1-\nu)^{2} r^{2} n^{2}+\nu \alpha^{2} \mu^{\prime 2}}{\left(n^{2}+\lambda_{i r}^{2}\right)\left(n^{2}+\lambda_{\beta r}^{2}\right)}=0 \\
(r, n=1,3,5 \ldots)
\end{array}
$$

$$
G_{\alpha n} \cong \frac{\left(\lambda_{\alpha n}^{2}-\nu \alpha^{2} n^{2}\right)^{2}}{\lambda_{\alpha n}} \mathfrak{I} \mathfrak{g} \frac{\pi}{2} \lambda_{\alpha_{n}}-\frac{\left(\lambda_{\alpha_{n}}^{\prime 2}-\nu \alpha^{2} n^{2}\right)^{2}}{\lambda_{\alpha n}^{\prime}} \mathfrak{I} \mathfrak{y} \frac{\pi}{2} \lambda_{\alpha_{n}}^{\prime} \cdot
$$

Analog erhält man aus den Randbedingungen 2)

mit

$$
\begin{array}{r}
b_{m}^{\prime}-\frac{8 \mu}{\pi G_{\beta m}} \sum_{s} a_{s}^{\prime} \frac{(1-\nu)^{2} m^{2} s^{2}+\nu \beta^{2} \mu^{2}}{\left(m^{2}+\lambda_{\alpha s}^{2}\right)\left(m^{2}+\lambda_{\alpha s}^{\prime 2}\right)}=0 \\
(m, s=1,3,5 \ldots)
\end{array}
$$

$$
\left.G_{\beta m} \equiv \frac{\left(\lambda_{i m}^{2}-\nu \beta^{2} m^{2}\right)^{2}}{\lambda_{\beta m}} \mathfrak{I} \mathfrak{g} \frac{\pi}{2} \lambda_{\beta m}-\frac{\left(\lambda_{i m}^{\prime 2}-\nu \beta^{2} m^{2}\right)^{2}}{\lambda_{\beta m}^{\prime}} \mathfrak{I g} \frac{\pi}{2} \lambda_{\beta m}^{\prime} \cdot\right)
$$

Die Elimination von $b_{r}^{\prime}$ aus (68) liefert

$$
a_{n}^{\prime}+\sum_{s} K_{n s} a_{s}^{\prime}=0, \quad(n, s=1,3,5 \ldots)
$$

mit

$$
K_{n s}=-\frac{64 \mu \mu^{\prime}}{\pi^{2} G_{\alpha_{n}}} \sum_{r} \frac{\left\{(1-\nu)^{2} r^{2} n^{2}+\nu \alpha^{2} \mu^{\prime 2}\right\}\left\{(1-\nu)^{2} r^{2} s^{2}+\nu \beta^{2} \mu^{2}\right\}}{G_{\beta r}\left(n^{2}+\lambda_{\beta r}^{2}\right)\left(n^{2}+\lambda_{\beta r}^{\prime 2}\right)\left(r^{2}+\lambda_{\alpha s}^{2}\right)\left(r^{2}+\lambda_{\alpha s}^{2}\right)} .
$$


Insbesondre für die quadratische Platte lässt sich schreiben

$$
K_{n s}=-\frac{64 \mu^{2}}{\pi^{2} G_{n}} \sum_{r} \frac{\left\{(1-\nu)^{2} r^{2} n^{2}+\nu \mu^{2}\right\}\left\{(1-\nu)^{2} r^{2} s^{2}+\nu \mu^{2}\right\}}{G_{r}\left\{\left(n^{2}+r^{2}\right)^{2}-\mu^{2}\right\}\left\{\left(r^{2}+s^{2}\right)^{2}-\mu^{2}\right\}}
$$

mit

$$
G_{n} \equiv \frac{\left(n^{2}-\nu n^{2}+\mu\right)^{2}}{\sqrt{n^{2}+\mu}} \mathfrak{I} \mathfrak{g} \frac{\pi}{2} \sqrt{n^{2}+\mu}-\frac{\left(n^{2}-\nu n^{2}-\mu\right)^{2}}{\sqrt{n^{2}-\mu}} \mathfrak{I} \mathfrak{g} \frac{\pi}{2} \sqrt{n^{2}-\mu}
$$

Der Ausdruck für $G_{r}$ ist derselbe wie der für $G_{n}$ mit $r$ an Stelle von $n$.

16. Die Formeln und die numerischen Rechenergebnisse für die qua. dratische Platte.

1) Die erste Schwingungsfamilie. Bei diesem Schwingungsfalle ist die Schwingungsfäche antisymmetrisch bzw. symmetrisch in bezug auf die beiden Mittellinien bzw. Diagonalen des Quadrats und wird gemäss $b_{n}^{\prime}\left(=b_{m}^{\prime}\right)=a_{n}^{\prime}$ durch

$$
\left.\begin{array}{l}
w^{\prime}=a_{1}^{\prime} \bar{w}^{\prime}, \\
\bar{w}^{\prime}=\sum_{n}(-1)^{\frac{n-1}{2}} \alpha_{n}^{\prime}\left\{v_{n}(\xi) \sin n \pi \eta+v_{n}(\eta) \sin n \pi \xi\right\}, \\
\quad a_{n}^{\prime}=\frac{a_{n}^{\prime}}{a_{1}^{\prime}}, \quad\left(\alpha_{1}^{\prime}=1\right), \quad(n=1,3,5 \ldots)
\end{array}\right\}
$$

ausgedrïckt. Da ferner

$$
\alpha=\beta=1, \quad \mu^{\prime}=\mu, \quad b_{r}^{\prime}=a_{s}^{\prime}
$$

ist, so erhält man aus (68)

$$
a_{n}^{\prime}-\frac{8 \mu}{\pi G_{n}} \sum_{n} a_{s}^{\prime} \frac{(1-\nu)^{2} n^{2} s^{2}+\nu \mu^{2}}{\left(n^{2}+s^{2}\right)^{2}-\mu^{2}}=0
$$

Somit vereinfacht sich der Ausdruck für $K_{n s}$, wie folgt:

$$
K_{n s}=-\frac{8 \mu}{\pi G_{n}} \frac{(1-\nu)^{2} n^{2} g^{2}+\nu \mu^{2}}{\left(n^{2}+s^{2}\right)^{2}-\mu^{2}} . \quad(n, s=1,3,5 \ldots)
$$

In analoger Weise wie in den früheren Fällen erhält man die in Tabelle 9 zusammengestellten Rechenergebnisse und die durch Abb. 22 dargestellten Knotenfiguren. Abb. 23 zeigt die entsprechenden Schichtlinien der $\bar{w}^{\prime}$-Fläche. 
Tabelle 9. Die Werte von $\mu$ und die der verschiedenen Beiwerte für die erste Familie der zweiten Schwingungsart der quadratischen Platte mit $\nu=0,3$.

\begin{tabular}{|c|c|c|c|c|c|c|c|}
\hline 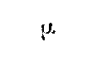 & $n$ & $\alpha_{n}$ & $\lambda_{n}$ & $\lambda^{2}{ }_{n}-v n^{2}$ & $x_{n}^{\prime}$ & $\lambda^{\prime 2} n-n^{2}$ & $\begin{array}{c}\text { Abb. } 22 . \\
\text { Knotenlinien. }\end{array}$ \\
\hline \multirow{4}{*}{ 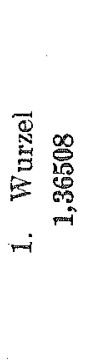 } & 1 & 1,000000 & 1,537881 & 2,06508 & $0,604219 i$ & $-0,66508$ & \\
\hline & 3 & 0,007663 & 3,219485 & 7,66508 & 2,763136 & 4,93492 & \\
\hline & 5 & 0,000997 & 5,134693 & 18,86508 & 4,861577 & 16,13492 & \\
\hline & 7 & 0,000406 & 7,096836 & 35,66508 & 6,901805 & 32,93492 & (I) \\
\hline \multirow{4}{*}{ 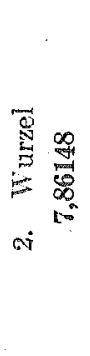 } & 1 & 1,000000 & 2,976849 & 8,56163 & $2,619471 i$ & $-7,16163$ & \\
\hline & 3 & 0,233391 & 4,106324 & 14.16163 & 1,066944 & $-1,56163$ & \\
\hline & 5 & 0,008881 & 5,732505 & 25,36163 & 4,139850 & 9,68837 & \\
\hline & 7 & 0,001778 & 7,540664 & 42,16163 & 6,413920 & 26,43837 & (II) \\
\hline \multirow{4}{*}{ 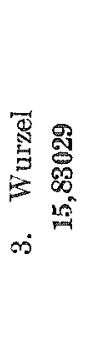 } & 1 & 1,000000 & 4,102474 & 16,53029 & $3,851012 i$ & $-15,13029$ & \\
\hline & 3 & $-4,560648$ & 4,982991 & 22,33029 & $2,613482 i$ & $-9,53029$ & \\
\hline & 5 & $-0,054907$ & 6,389858 & 33,33029 & 3,028153 & 1,66971 & \\
\hline & 7 & $-0,014567$ & 8,051760 & 50,13029 & 5,759315 & 18,46971 & (III) \\
\hline \multirow{4}{*}{ 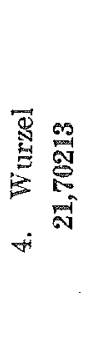 } & 1 & 1,000000 & 4,764675 & 22,40213 & $4,549959 i$ & $-21,00213$ & \\
\hline & 3 & $-0,076128$ & 5,540950 & 28,00213 & $3,564005 i$ & $-15,40213$ & \\
\hline & 5 & $0,17938.1$ & 6,833894 & 39,20213 & 1,816004 & - 4,20213 & \\
\hline & 7 & 0,011812 & 8,408461 & 56,00213 & 5,224735 & 12,59787 & (IV) \\
\hline
\end{tabular}




\begin{tabular}{|c|c|c|c|c|c|c|c|}
\hline$\mu$ & $n$ & $\alpha_{n}$ & $\lambda_{n}$ & $\lambda n^{2}-v n^{2}$ & $\lambda_{n}^{\prime}$ & $\lambda^{\prime 2} n-v n^{2}$ & $\begin{array}{c}\text { Abb. } 12 . \\
\text { Knotenlinien. }\end{array}$ \\
\hline \multirow{4}{*}{ 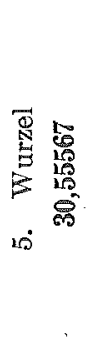 } & 1 & 1,000000 & 5,617444 & 31,25567 & $5,436513 i$ & $-29,85567$ & \\
\hline & 3 & $-6,105806$ & 6,289330 & 36,85567 & $4,642808 i$ & $-24,25567$ & \\
\hline & 5 & $-2,801751$ & 7,453567 & 48,05567 & $2,357046 i$ & $-13,05567$ & \\
\hline & 7 & $-0,122306$ & 8,919400 & 64,85567 & 4,294686 & 3,74433 & (V) \\
\hline
\end{tabular}

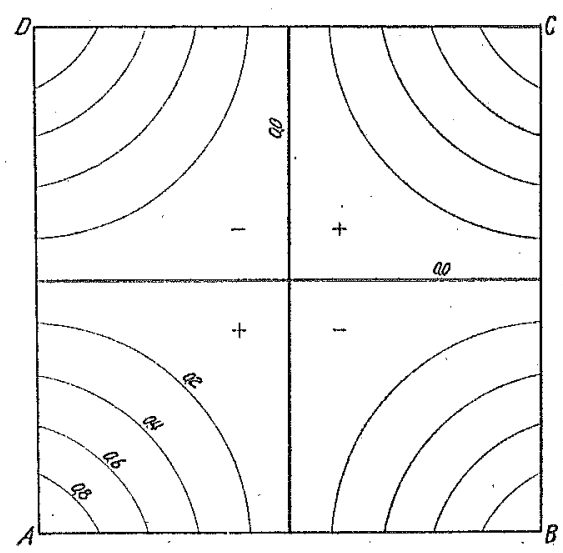

Schnitt $A-B$

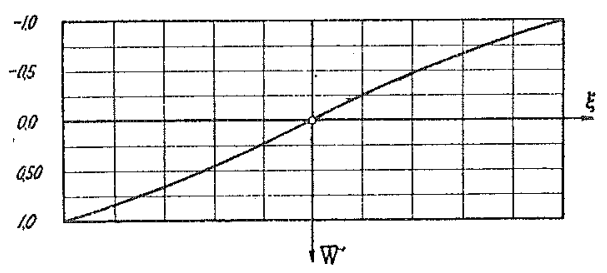

(I), $\mu_{1}=1,36508$.

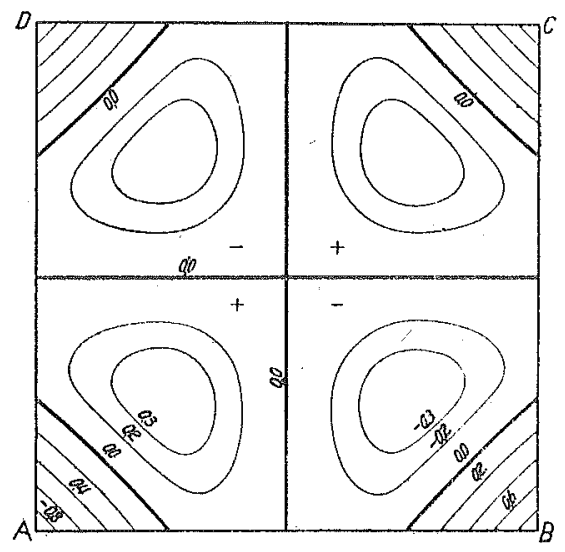

Sotnitt $A-B$

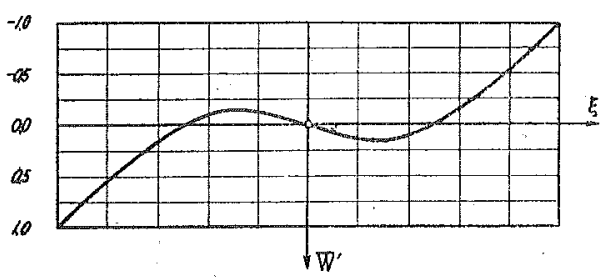

(II), $\mu_{2}=7,86148$. 
Die Eigenschwingungen und Klangfiguren der viorseitig freien rechteckigen Platte.
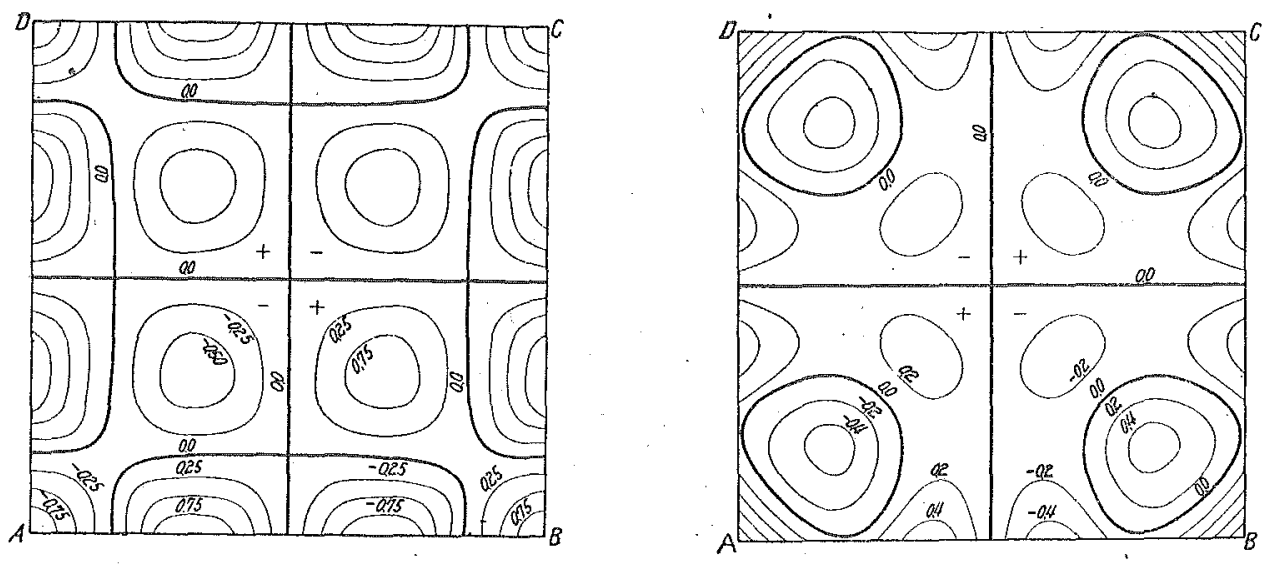

Schitt $A-B$

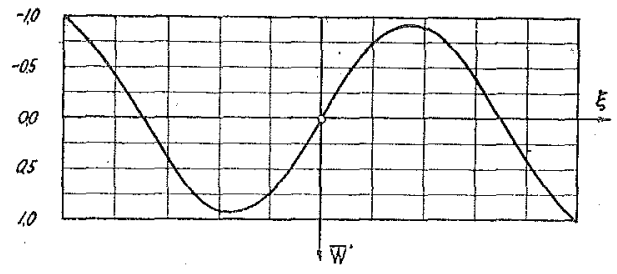

(III), $\mu_{3}=15,83029$.

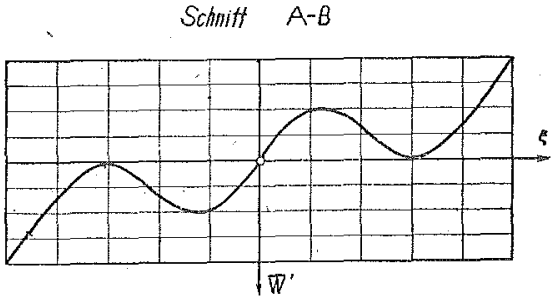

(IV), $\mu_{4}=21,70213$

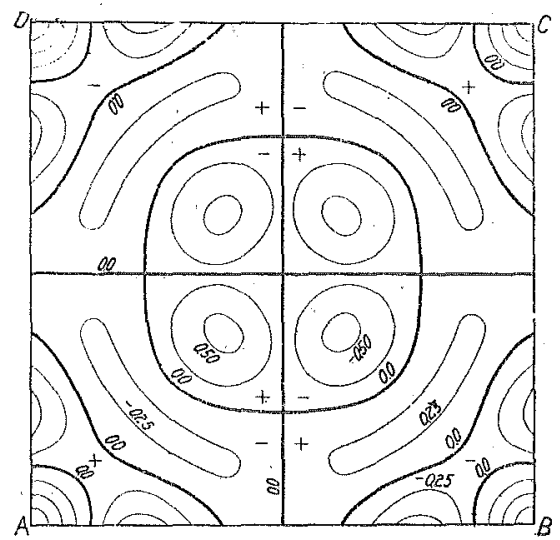

Schnitt $A-B$

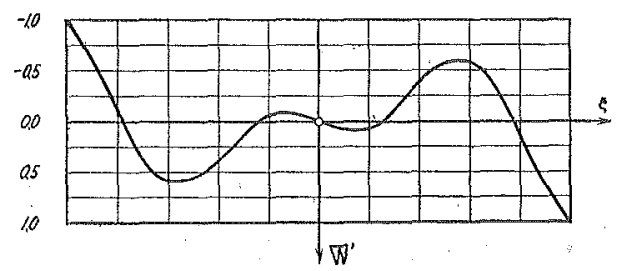

(V) $, \mu_{5}=30,55567$.

Abb. 23. Schichtlinien der $\bar{w}^{\prime}-$ Fläche für die erste Familie der zweiten Schwingungsart. 
Die in Tabelle 9 angeschrièbene erste Wurzel $\mu_{1}=1,36508$ ist der allerniedrigste Eigenwert für alle Schwingungsarten der vierseitig freien quadratischen Platte ohne Eckstütze, also gehört sie dem Grundton an, in welchem die Platte mit den beiden Mittellinien $\xi=0$ und $\eta=0$ als ihre Knotenlinien schwingt, wie Abb. 22-(I) oder 23-(I) zeigt.

2) Die zweite Schwingungsfamilie. Wir wollen uns jetzt mit der zy̧eiten Familie der zweiten Schwingungsart beschäftigen, in der die Schwingungfläche sich antisymmetrisch nicht nur in bezug auf die beiden Mittellinien, sondern auch auf die beiden Diagonalen des Quadrats gestaltet. Die Schwingungsfïche hat hierbei mindestens vier Knotenlinien $\xi=0, \eta=0, \xi=\eta, \xi=-\eta$, und der Ausdruck für $\bar{w}^{\prime}$ wird nur durch Umkehrung des Vorzeichens von $v_{n}(\eta)$ in (72) ermittelt. Also lautet

$$
\begin{gathered}
\bar{w}^{\prime}=\sum_{n}(-1)^{\frac{n-1}{2}} a_{n}^{\prime}\left\{v_{n}(\xi) \sin n \pi \eta-v_{n}(\eta) \sin n \pi \xi\right\} . \\
(n=1,3,5 \ldots)
\end{gathered}
$$

Analog ist der Ausdruck für $K_{n s}$ derselbe wie der in (73) mit entgegengesetztem Vorzeichen.

Der allgemeine Ausdruck von $K_{n s}(71)$ ist verschieden von dem von $K_{n s}$ (73) für die erste Familie sowie des $-K_{n s}$ (73) für die zweite Familie. Aber man kann ohne grosse Schwierigkeit beweisen, dass die durch $K_{n s}(71)$ gebildete Frequenzgleichung gleichzeitig die Wurzeln für die beiden Familio deswegen liefert, weil diese Frequenzgleichung mit der aus dem Produkt der einzelnen Frequenzgleichung für jede Familie hervorgehenden Gleichung identisch ist.

Die numerischen Rechenergebnisse für die ersten vier Wurzeln sind in Tabelle 10 zusammengestellt, während die entsprechenden Knotenfiguren durch Abb. 24 gezeigt werden. Abb. 25 stellt die Schichtlinien der zu jeder Wurzel gehörenden $\bar{w}^{\prime}-$ Fläche dar.

Tabelle 10. Die Werte von $\mu$ und die der verschiedenen Beiwerte für die zweite Familie der zweiten Schwingungsart der

\begin{tabular}{|c|c|c|c|c|c|c|c|}
\hline$\mu$ & $n$ & $\alpha_{n}$ & $\lambda_{n}$ & $\lambda^{2} n-v n^{2}$ & $\lambda n_{n}$ & $\gamma^{\prime 2} n-\mathrm{v} n^{2}$ & $\begin{array}{c}\text { Abb. } 24 . \\
\text { Knotenlinien. }\end{array}$ \\
\hline \multirow{4}{*}{ 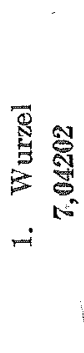 } & 1 & 1,000000 & 2,835846 & 7,74202 & $2,458051 i$ & $-6,34202$ & \\
\hline & 3 & $-0,128266$ & 4,005250 & 13,34202 & 1,399278 & $-0,64202$ & \\
\hline & 5 & $-0,005566$ & 5,660567 & 24,54202 & 4,237686 & 10,45798 & \\
\hline & 7 & $-0,001010$ & 7,486121 & 41,34202 & 6,477499 & 27,25798 & (I) \\
\hline
\end{tabular}
quadratischen Platte mit $\nu=0,3$. 
Die Eigenschwingungen und Klangfiguren der vierseitig freien rechteckigen Platte.

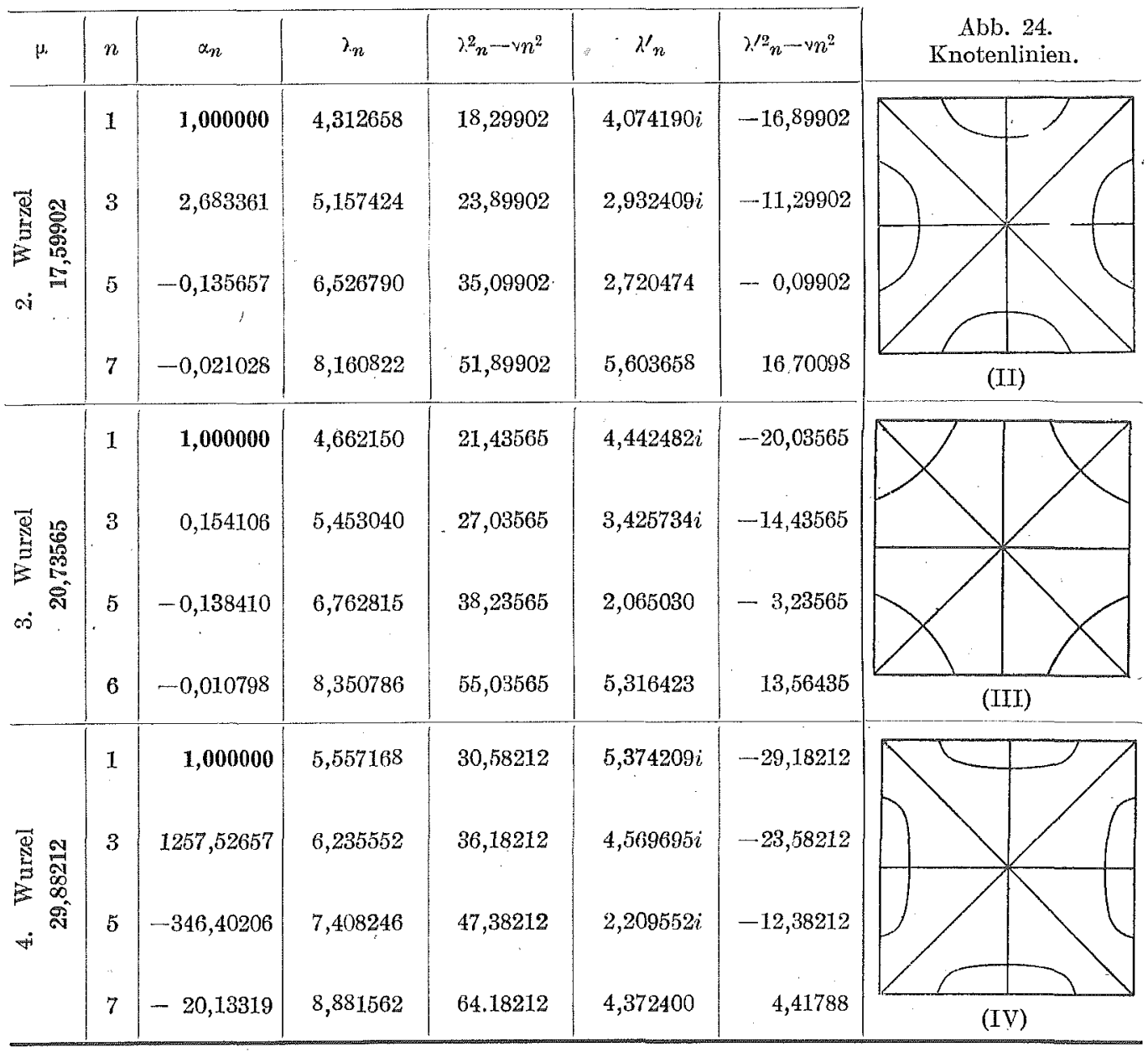



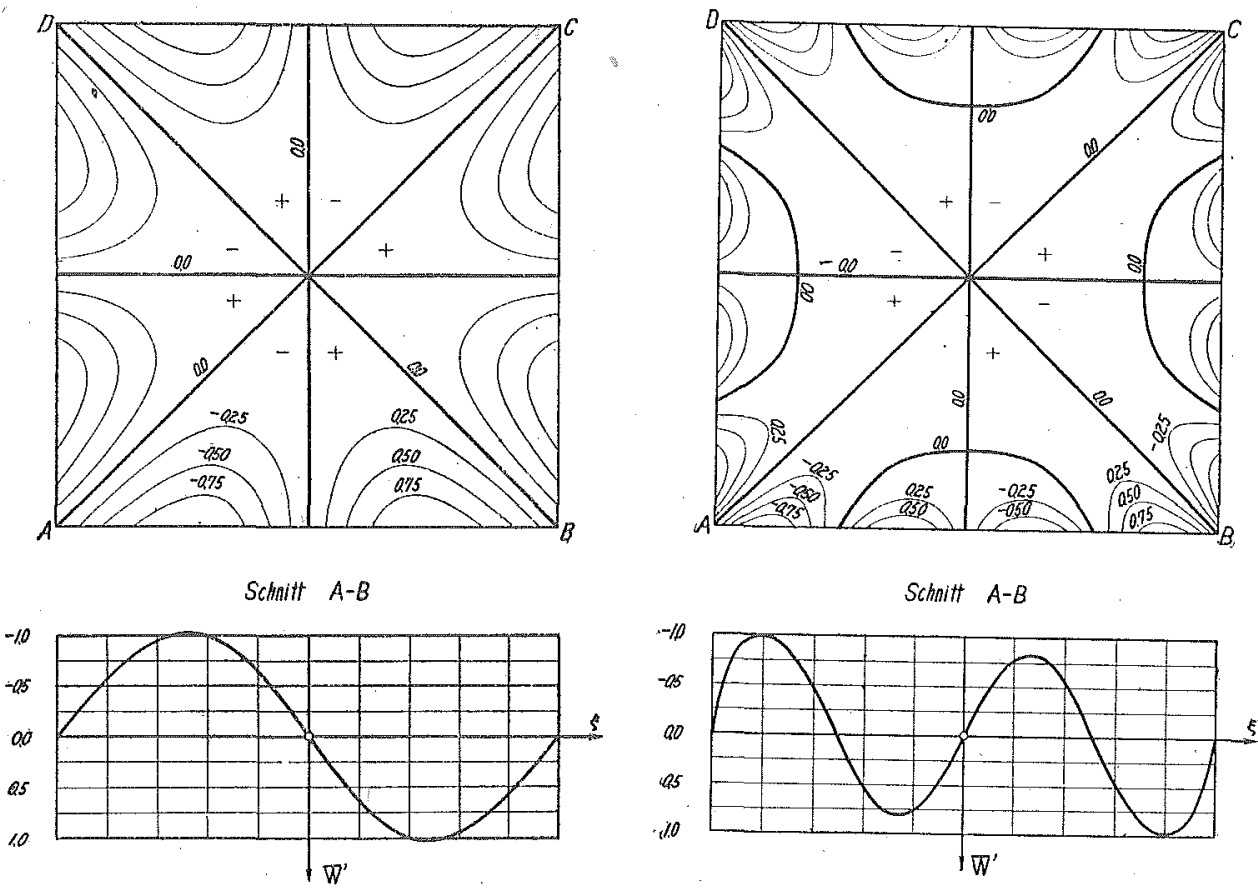

(I) $\mu_{1}=7,04202$.

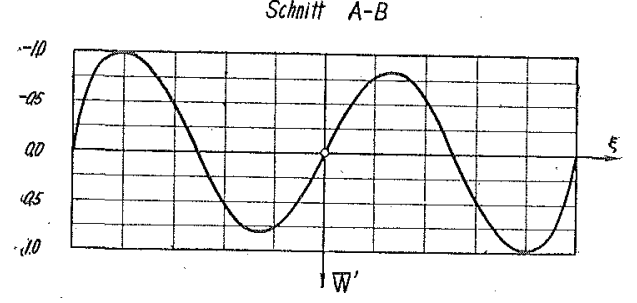

(TI), $\mu_{2}=17,59902$.
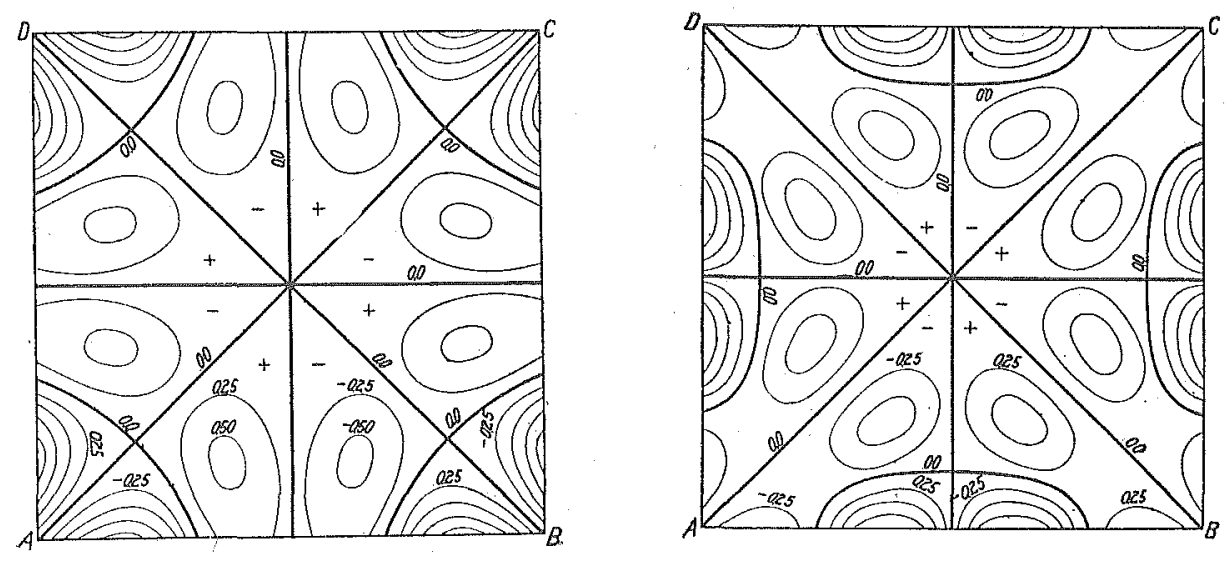

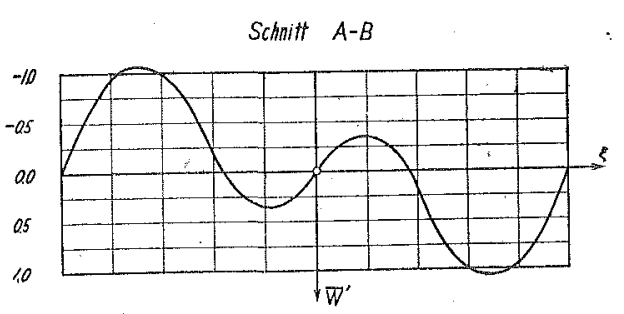

(III). $\mu_{3}=20,73565$.

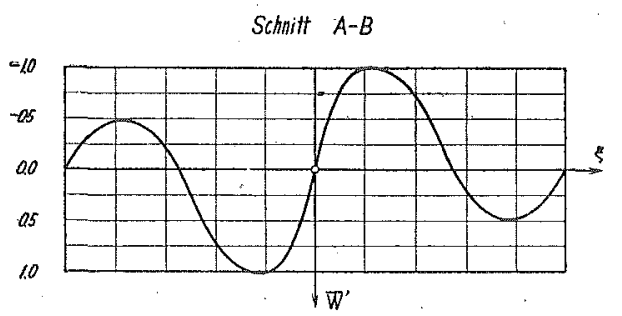

(IV), $\mu_{4}=29,88212$.

Abb. 25. Schichtlinien der $\bar{u}-$ Fläche für die zweite Familie der zweiten Schwingungsart. 


\section{Die dritte Schwingungsart.}

17. Die allgemeinen Formeln für die rechteckige Platte. Wir wollen hierbei dio dritten Schwingungsart betrachten, in welcher dio Auslenkung einer beliebig durch den Mittelpunkt des Rechtecks gezogenen geraden Linie antisymmetrisch in bezug auf dieson Punkt ist. Der einfachste Fall mit dieser Eigenschaft ist der, in dem die Schwingungsfläche symmetrisch in bezug auf die eine von den beiden Mittellinien, antisymmetrisch dagegen in bezug auf die andore ist. Wenn es sich erstens um die Schwingungsfläche $w_{a}^{\prime}$ handelt, die symmetrisch bzw. antisymmetrisch in bezug auf die Mittellinie $\xi=0$ bzw. $\eta=0$ ist, so hat sie mindestens eine Knotenlinie $\eta=0$ und wird durch

mit

$$
\begin{gathered}
w_{a}^{\prime}=\sum_{n}(-1)^{\frac{n-1}{2}} a_{n} u_{\alpha_{n}}(\xi) \sin n \pi \eta+b_{0}^{\prime} v_{0}(\eta)+\sum_{m}(-1)^{\frac{m}{2}} b_{m}^{\prime} v_{\beta m}(\eta) \cos m \pi \xi \\
(m=2,4,6 \ldots, n=1,3,5 \ldots)
\end{gathered}
$$

$$
v_{0}(\eta)=\lim _{m \rightarrow 0} v_{\beta m}(\eta)=\sqrt{\mu^{\prime}}\left(\frac{\operatorname{Sin} \pi \sqrt{\mu^{\prime} \eta}}{\operatorname{Soj} \frac{\pi}{2} \sqrt{\mu^{\prime}}}+\frac{\sin \pi \sqrt{\mu^{\prime} \eta}}{\cos \frac{\pi}{2} \sqrt{\mu^{\prime}}}\right)
$$

ausgedrückt.

In ähnlicher Weise wie in den früheren Fällen ergibt sich aus den Randbedingungen 1)

$$
\begin{array}{r}
a_{n}-\frac{8 \mu^{\prime}}{\pi H_{\alpha n}}\left\{b_{0}^{\prime} \frac{\nu \alpha^{2} \mu^{\prime 2}}{n^{4}-\mu^{\prime 2}}+\sum_{r} b_{r}^{\prime} \frac{(1-\nu)^{2} r^{2} n^{2}+\nu \alpha^{2} \mu^{\prime 2}}{\left(n^{2}+\lambda_{\beta r}^{2}\right)\left(n^{2}+\lambda_{\beta r}^{\prime 2}\right)}\right\}=0 . \\
(r=2,4,6 \ldots, \quad n=1,3,5 \ldots)
\end{array}
$$

Hierin ist der Ausdruck für $H_{\alpha_{n}}$ derselbe wie der in (56). Analog erhält man aus den Randbedingungen 2)

$$
\begin{aligned}
& b_{m}^{\prime}-\frac{8 \mu}{\pi G_{\beta m}} \sum_{s} a_{s} \frac{(1-\nu)^{2} m^{2} s^{2}+\nu \beta^{2} \mu^{2}}{\left(m^{2}+\lambda_{\alpha_{s}}^{2}\right)\left(m^{2}+\lambda_{\alpha_{s}}^{\prime 2}\right)}=0, \\
& \quad(m=2,4,6 \ldots, \quad s=1,3,5 \ldots) \\
& b_{0}^{\prime}=\frac{4 \nu \mu^{2}}{\pi t^{\prime}} \sum_{s} a_{s} \frac{\mu^{\prime 2}}{s^{4}-\mu^{\prime 2}}
\end{aligned}
$$

mit

$$
t^{\prime}=\lim _{m \rightarrow 0} G_{\beta m} \equiv \mu^{\prime} \sqrt{\mu^{\prime}}\left(\mathfrak{I} \mathfrak{g} \frac{\pi}{2} \sqrt{\mu^{\prime}}-\operatorname{tg} \sqrt{ } \overline{\mu^{\prime}}\right)
$$

Setzt man den Ausdruck für $b_{0}^{\prime}$ in (77) und den aus der ersten Gleichung in (77) hervorgehenden Ausdruck für $b_{r}^{\prime}\left(=b_{m}^{\prime}\right)$ in (76) ein, so erhält man

mit

$$
a_{n}+\sum_{s} K_{n s} a_{s}=0, \quad(n, s=1,3,5 \ldots)
$$




$$
\left.\begin{array}{rl}
K_{n s}= & -\frac{64 \mu \mu^{\prime}}{\pi^{2} H_{\alpha_{n}}}\left\{\frac{\nu^{2} \mu^{\prime 4}}{2 t^{\prime}\left(n^{4}-\mu^{\prime 2}\right)\left(s^{4}-\mu^{\prime 2}\right)}\right. \\
& \left.+\sum_{r} \frac{\left\{(1-\nu)^{2} r^{2} n^{2}+\nu \alpha^{2} \mu^{\prime 2}\right\}\left\{(1-\nu)^{2} r^{2} s^{2}+\nu \beta^{2} \mu^{2}\right\}}{G_{\beta r}\left(n^{2}+\lambda_{\beta r}^{2}\right)\left(n^{2}+\lambda_{i r}^{\prime 2}\right)\left(r^{2}+\lambda_{\alpha s}^{2}\right)\left(r^{2}+\lambda_{\alpha s}^{\prime 2}\right)}\right\}
\end{array}\right\}
$$

Daraus kann man leicht die Frequenzgleichung ableiten.

Wenn es sich zweitens um den Fall handelt, in dem die Schwingungsfläche antisymmetrisch bzw. symmetrisch in bezug auf die Mittellinie $\xi=0$ bzw. $\eta=0$ des Rechtecks ist, so erhält man ohne weiteres

$$
\begin{gathered}
w_{b}^{\prime}=a_{0}^{\prime} v_{0}(\xi)+\sum_{n}(-1)^{\frac{n}{2}} a_{n}^{\prime} v_{\alpha_{n}}(\xi) \cos n \pi \eta+\sum_{m}(-1)^{\frac{m-1}{2}} b_{m} u_{\beta m}(\eta) \sin m \pi \xi \\
(m=1,8,5 \ldots, \quad n=2,4,6 \ldots)
\end{gathered}
$$

mit

$$
v_{0}(\xi)=\lim _{n \rightarrow 0} v_{\alpha_{n}}(\xi)=\gamma_{\mu}\left(\frac{\operatorname{Sin} \pi \sqrt{\mu} \xi}{\operatorname{Cof} \frac{\pi}{2} \sqrt{\mu}}+\frac{\sin \pi \sqrt{\mu} \xi}{\operatorname{Cos} \frac{\pi}{2} \sqrt{\mu}}\right) .
$$

Dafür gilt die Gleichung für $b_{m}$, wie folgt: *

mit

$$
b_{m}+\sum_{r} K_{m r} b_{r}=0, \quad(m, r=1,3,5 \ldots)
$$

$$
\begin{aligned}
& K_{m r}=-\frac{64 \mu \mu^{\prime}}{\pi^{2} I_{\beta m}}\left\{\frac{\nu^{2} \mu^{4}}{2 t\left(m^{4}-\mu^{2}\right)\left(r^{4}-\mu^{2}\right)}\right. \\
&\left.+\sum_{s} \frac{\left\{(1-\nu)^{2} m^{2} s^{2}+\nu \beta^{2} \mu^{2}\right\}\left\{(1-\nu)^{2} r^{2} s^{2}+\nu \alpha^{2} \mu^{\prime 2}\right\}}{G_{\alpha_{s}}\left(m^{2}+\lambda_{\alpha_{s}}^{2}\right)\left(m^{2}+\lambda_{\alpha s}^{\prime 2}\right)\left(s^{2}+\lambda_{\beta r}^{2}\right)\left(s^{2}+\lambda_{\beta r}^{\prime 2}\right)}\right\} \\
& t=\lim _{n \rightarrow 0} G_{\alpha_{n}} \equiv \mu \sqrt{\mu}\left(\operatorname{Iq}_{\mathrm{q}} \frac{\pi}{2} \sqrt{\mu}-\operatorname{tg} \frac{\pi}{2} \sqrt{\mu}\right)
\end{aligned}
$$

\section{Die Formeln und die numerischen Rechenergebnisse für die quadrati. sche Platte.}

1) Dio erste Schwingungsart. 'Wir wollen jetzt den Fall betrachten, in dem die Platte symmetrisch bzw. antisymmetrisch in bezug auf die Mittellinie $\xi=0$ bzw. $\eta=0$ des Quadrats schwingt. Die Fläche hierfür lautet sofort nach (75)

$$
\begin{gathered}
w_{a}^{\prime}=a_{1} \bar{w}_{a}^{\prime}, \\
\bar{w}_{a}^{\prime}=\sum_{n}(-1)^{\frac{n-1}{2}} a_{n} u_{n}(\xi) \sin n \pi \eta+\beta_{0}^{\prime} v_{0}(\eta)+\sum_{m}(-1)^{\frac{m}{2}} \beta_{m}^{\prime} v_{m}(\eta) \cos m \pi \xi \\
(m=2,4,6 \ldots, \quad n=1,3,5 \ldots), \\
a_{n}=\frac{a_{n}}{a_{1}}, \quad\left(\alpha_{1}=1\right), \quad \beta_{0}^{\prime}=\frac{b_{0}^{\prime}}{a_{1}}, \quad \beta_{m}^{\prime}=\frac{b_{m}^{\prime}}{a_{1}} .
\end{gathered}
$$

Analog für die antisymmetrisch bzw. symmetrisch in bezug auf die Mittellinie $\xi=0$ bzw. $\eta=0$ schwingende Fläche ergibt sich aus (79) 


$$
\begin{aligned}
& w_{b}^{\prime}=b_{1} \bar{w}_{b}^{\prime}, \\
& \bar{w}_{b}^{\prime}=\alpha_{0}^{\prime} v_{0}(\xi)+\sum_{n}(-1)^{\frac{n}{2}} \alpha_{n}^{\prime} v_{n}(\xi) \cos n \pi \eta+\sum_{m}(-1)^{\frac{m \cdot 1}{2}} \beta_{m} u_{m}(\eta) \sin m \pi \xi, \\
& (m=1,3,5 \ldots, n=2,4,6 \ldots) \\
& \alpha_{0}^{\prime}=\frac{a_{0}^{\prime}}{b_{1}}, \quad \alpha_{n}^{\prime}=\frac{a_{n}^{\prime}}{b_{1}}, \quad \beta_{m}=\frac{b_{m}}{b_{1}}, \quad\left(\beta_{1}=1\right) .
\end{aligned}
$$

Wie in Ziff. 10 für die quadratische Platte mit Eckstützen erwähnt, sind die Grössen von $\mu$ für $\bar{u}_{b}^{\prime}$ gleich den entsprechenden Grössen von $\mu$ für $\bar{u}_{a}^{\prime}$. Die Werte von $\mu$ für die beiden Schwingungsflächen $\bar{w}_{a}^{\prime}$ und $\bar{w}_{b}^{\prime}$ können daher durch Auflösung der durch $K_{n s}$ (78) mit $\alpha=\beta=1$, nämlich durch

$$
K_{n s}=-\frac{64 \mu^{2}}{\pi^{2} H_{n}}\left\{\frac{\nu^{2} \mu^{4}}{2 t\left(n^{4}-\mu^{2}\right)\left(s^{4}-\mu^{2}\right)}+\sum_{r} \frac{\left\{(1-\nu)^{2} \gamma^{2} n^{2}+\nu \mu^{2}\right\}\left\{(1-\nu)^{2} r^{2} s^{2}+\nu \mu^{2}\right\}}{G_{r}\left\{\left(n^{2}+r^{2}\right)^{2}-\mu^{2}\right\}\left\{\left(r^{2}+s^{2}\right)^{2}-\mu^{2}\right\}}\right\}
$$

gebildeten Frequenzgleichung ermittelt werden.

Die wirkliche Durchrechnung liefert die in Tabelle 11 zusammengestellten Ergebnisse. Die in Abb. 26 mit dem Zeichen (I-1), (II-1), (III-1) bzw. (IV-1) gezeigten Figuren sind die der ersten, zweiten, dritten bzw. vierten Wurzel angehörenden Knotenlinien, während die entsprechenden Schichtlinien der $\bar{w}_{a}^{\prime}-$ Fläche in Abb. 27 dargestellt werden.

2) Die zweite Schwingungsfamilie. Es handelt sich jetzt um die zweite Familie der dritton Schwingungsart, in der die Platte antisymmetrisch in bezug auf eine Diagonale des Quadrats schwingt. Hierbei hat die Schwingungsfläche mindestens eine Diagonale als Knotenlinie und ergibt sich aus (81) und (82)

$$
\bar{w}^{\prime}=\bar{w}_{a}^{\prime} \pm \bar{w}_{b}^{\prime}
$$

Offenbar hat jede Teilschwingung $\bar{w}_{\alpha}^{\prime}$ und $\bar{w}_{b}^{\prime}$, und folglich auch die resultierende Schwingung eine und dieselbe Schwingungsdauer. Die Fläche $\bar{w}_{a}^{\prime}+\bar{u}_{b}^{\prime}$ und $\bar{w}_{a}^{\prime}-\bar{w}_{b}^{\prime}$ hat je eine verschiedene Diagonale als Kriotenlinie. Die in Abb. 26 mit den Zeichen (I-4), (II-4), (III-4), (IV-4) gezeigten Figuren stellen die den ersten bis vierten Wurzeln $\mu$ entsprechenden Knotenlinien $\bar{w}^{\prime}=\bar{u}_{a}^{\prime}-\bar{w}_{b}^{\prime}=0$ für die quadratische Platte dar, während die entsprechenden Schichtlinien der $\bar{w} \bar{w}^{\prime}$-Fläche in Abb. 28 gezeigt werden.

3) Die dritte Schwingungsfamilie. Da die Schwingungsdauern von $\bar{w}_{a}^{\prime}$ und $\bar{w}_{b}^{\prime}$ gleich untereinander sind, so kann man die beiden Flächen linear so verbinden, dass

$$
\bar{w}^{\prime}=\bar{w}_{a}^{\prime}+\kappa \bar{w}_{b}^{\prime}
$$

Hierin bedeutet $\kappa$ eine beliebige reelle Zahl. Man nennt wie früher $\overline{v i}^{\prime}(85)$ mit $0<|\kappa|<1$ die dritte Schwingungsfamilie, und die erste bzw. zweite Familie entspricht dem Sonderwert $\kappa=0,0$ bzw. $\kappa= \pm 1,0$. 
Tabelle. 11. Die $W$ erte von $\mu$ und die der verschiedenen Beiwerte für die dritte Schwingungsart der quadratisehen Platte mit $\nu=0,3$.

\begin{tabular}{|c|c|c|c|c|c|c|c|c|c|c|c|c|}
\hline$\mu$ & $n$ & $\alpha_{n}$ & $\lambda_{n}$ & $i_{n}-n^{2}$ & $Y_{n}^{\prime}$ & $x_{n}^{\prime}-n^{2}$ & $m$ & $\beta_{m}^{\prime}$ & $\lambda \cdot m$ & $\lambda:_{m}^{2}-v m^{2}$ & $X_{m}^{\prime}$ & $\lambda_{m}^{\prime}-v m^{2}$ \\
\hline 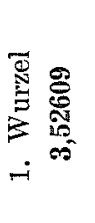 & $\begin{array}{l}1 \\
3 \\
5 \\
7\end{array}$ & $\begin{array}{c}- \\
1,000000 \\
0,011819 \\
0,004296 \\
0,001827\end{array}$ & $\begin{array}{c}- \\
2,127460 \\
3,539221 \\
5,340982 \\
7,247489\end{array}$ & $\begin{array}{c}- \\
4,22609 \\
9,82609 \\
21,02609 \\
37,82609\end{array}$ & \begin{tabular}{l}
\multicolumn{1}{c}{-} \\
$1,589368 i$ \\
2,339639 \\
4,633994 \\
6,743434
\end{tabular} & $\begin{array}{c}- \\
-2,82609 \\
2,77391 \\
13,97391 \\
30,77391\end{array}$ & $\begin{array}{l}0 \\
2 \\
4 \\
6 \\
8\end{array}$ & $\begin{array}{r}-0,185678 \\
0,292184 \\
0,012179 \\
0,003208 \\
0,001325\end{array}$ & $\begin{array}{c} \\
2,743372 \\
4,418835 \\
6,286978 \\
8,217425\end{array}$ & $\begin{array}{r}- \\
6,32609 \\
14,72609 \\
28,72609 \\
48,32609\end{array}$ & $\begin{array}{c}- \\
0,688411 \\
3,531843 \\
5,698587 \\
7,776498\end{array}$ & $\begin{array}{r}- \\
-0,72609 \\
7,67391 \\
21,67391 \\
41,27391\end{array}$ \\
\hline 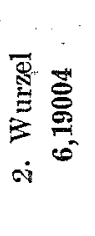 & $\begin{array}{l}1 \\
3 \\
5 \\
7\end{array}$ & $\begin{array}{c}- \\
1,000000 \\
-0,860284 \\
-0,043478 \\
-0,009735\end{array}$ & $\begin{array}{c}- \\
2,681449 \\
3,897457 \\
5,584816 \\
7,429008\end{array}$ & $\begin{array}{c}- \\
6,89017 \\
12,49017 \\
23,69017 \\
40,49017\end{array}$ & $\begin{array}{c}- \\
2,278194 i \\
1,676255 \\
4,337031 \\
6,542922\end{array}$ & \begin{tabular}{r}
\multicolumn{1}{c}{-} \\
$-5,49017$ \\
0,10983 \\
11,30983 \\
28,10983
\end{tabular} & $\begin{array}{l}0 \\
2 \\
4 \\
6 \\
8\end{array}$ & $\begin{array}{l}-7,629322 \\
-0,565877 \\
-0,013918 \\
-0,010758 \\
-0,005946\end{array}$ & $\begin{array}{c}- \\
3,192205 \\
4,710645 \\
6,495396 \\
8,377956\end{array}$ & $\begin{array}{c}- \\
8,99017 \\
17,39017 \\
31,39017 \\
50,99017\end{array}$ & \begin{tabular}{l}
\multicolumn{1}{c}{-} \\
$1,479922 i$ \\
3,132067 \\
5,459838 \\
7,603278
\end{tabular} & $\begin{array}{r}- \\
-3,39017 \\
5,00983 \\
19,00983 \\
38,60983\end{array}$ \\
\hline 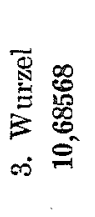 & $\begin{array}{l}1 \\
3 \\
5 \\
7\end{array}$ & $\begin{array}{c}- \\
1,000000 \\
-1,433111 \\
-0,077880 \\
-0,023908\end{array}$ & $\begin{array}{c}- \\
3,418433 \\
4,436854 \\
5,973749 \\
7,725652\end{array}$ & $\begin{array}{c}- \\
11,38568 \\
16,98568 \\
28,18568 \\
44,98568\end{array}$ & $\begin{array}{l}- \\
3,112183 i \\
1.298337 i \\
3,783427 \\
6,189855\end{array}$ & $\begin{array}{r}- \\
-9,98568 \\
-4,38568 \\
6,81432 \\
23,61432\end{array}$ & $\begin{array}{l}0 \\
2 \\
4 \\
6 \\
8\end{array}$ & $\begin{array}{r}0,141058 \\
-3,038828 \\
-0,019728 \\
-0,021659 \\
-0,011355\end{array}$ & $\begin{array}{c}- \\
3,832190 \\
5,165819 \\
6,832691 \\
8,642087\end{array}$ & $\begin{array}{c}- \\
13,48568 \\
21,88568 \\
35,88568 \\
55,48568\end{array}$ & \begin{tabular}{c}
\multicolumn{1}{c}{-} \\
$2,585668 i$ \\
2,305281 \\
5,031334 \\
7,301668
\end{tabular} & \begin{tabular}{r}
\multicolumn{1}{c}{-} \\
$-7,88568$ \\
0,51432 \\
14,51432 \\
34,21432
\end{tabular} \\
\hline 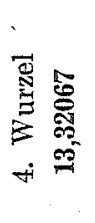 & $\begin{array}{l}1 \\
3 \\
5 \\
7\end{array}$ & $\begin{array}{c}- \\
1,000000 \\
0,093333 \\
0,002501 \\
0,002445\end{array}$ & $\begin{array}{c}- \\
3,784266 \\
4,724476 \\
6,190368 \\
7,894345\end{array}$ & $\begin{array}{c}- \\
14,02067 \\
19,62067 \\
30,82067 \\
47,62067\end{array}$ & \begin{tabular}{l}
\multicolumn{1}{c}{-} \\
$3,510081 i$ \\
$2,078631 i$ \\
3,417504 \\
5,973218
\end{tabular} & $\begin{array}{r}- \\
12,62067 \\
-7,02067 \\
4,17983 \\
20,97933\end{array}$ & $\begin{array}{l}0 \\
2 \\
4 \\
6 \\
8\end{array}$ & $\begin{array}{r}-0,076301 \\
-0,078150 \\
0,179722 \\
0,013230 \\
0,003816\end{array}$ & $\begin{array}{c}- \\
4,161811 \\
5,414857 \\
7,022868 \\
8,793216\end{array}$ & $\begin{array}{c}- \\
16,12067 \\
24,52067 \\
38,52067 \\
58,12067\end{array}$ & $\begin{array}{c}- \\
3,052977 i \\
1,636866 \\
4,762270 \\
7,118942\end{array}$ & $\begin{array}{r}- \\
-10,52067 \\
-2,12067 \\
11,87933 \\
31,47933\end{array}$ \\
\hline
\end{tabular}


Die Eigenschwingungen und Klangfiguren der vierseitig freien rechteckigen Platte.

1. Wurzel $\left(\mu_{1}\right)$

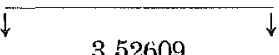

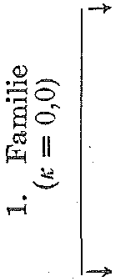

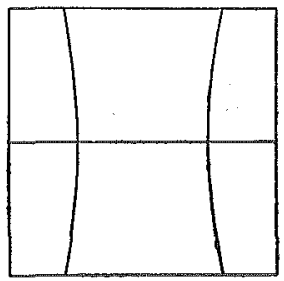

$(\mathrm{I}-1)$
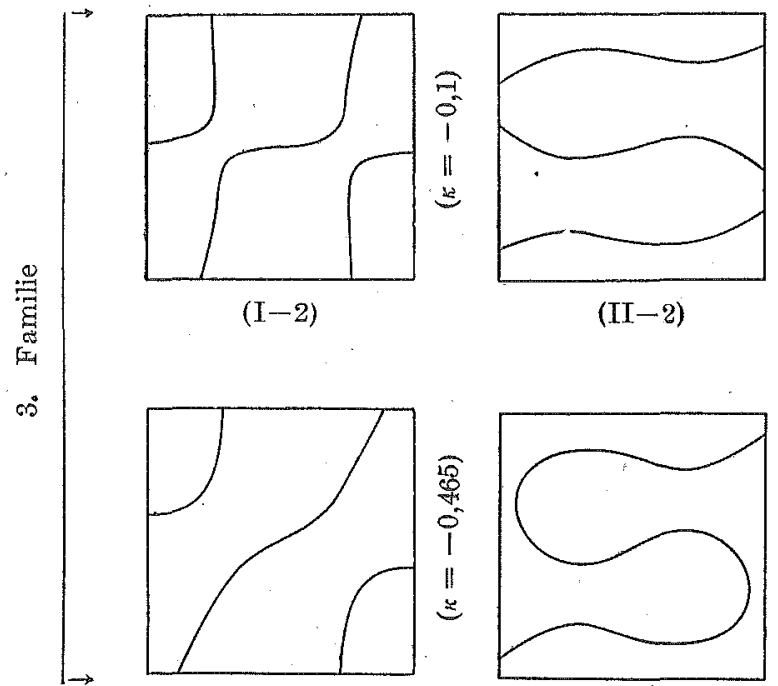

$(\mathrm{I}-3)$

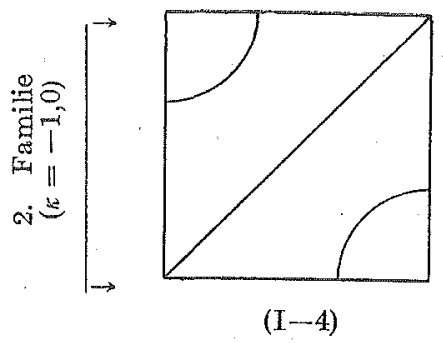

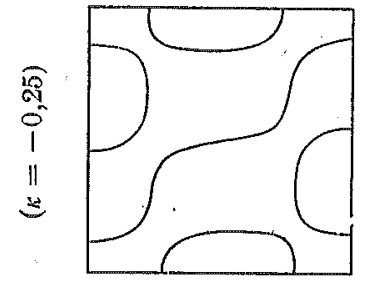

(IIII-2)

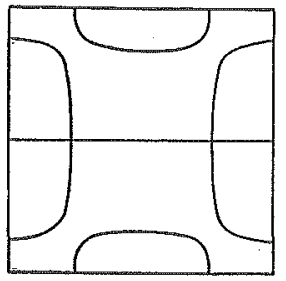

(III-1)

(II -1$)$

$(\mathrm{II}-2)$

3. Wurzel $\left(\mu_{3}\right)$

$\downarrow \quad \downarrow 10,68568 \quad \downarrow$
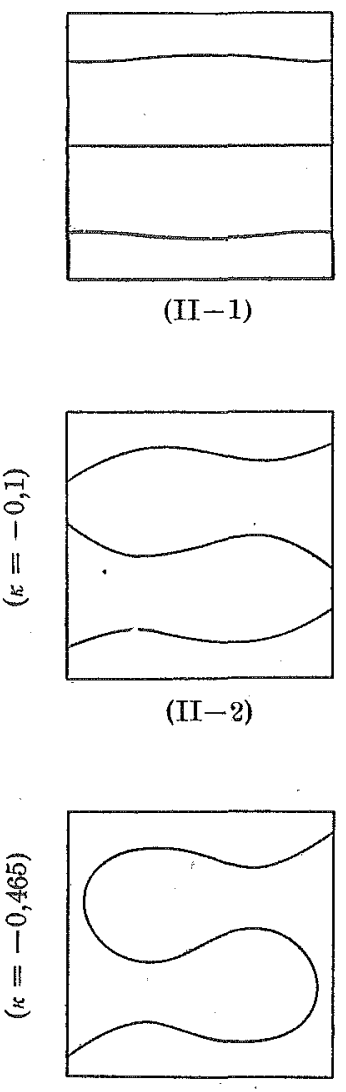

(II-3)

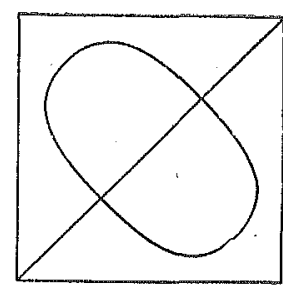

(II-4)

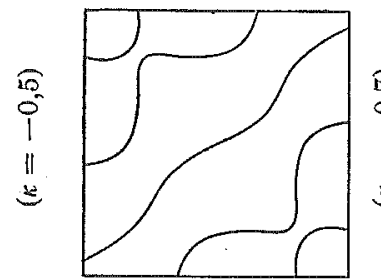

(III-3)

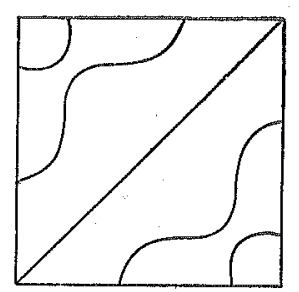

(III-4)
4. Wurzel $\left(\mu_{\cdot 4}\right)$
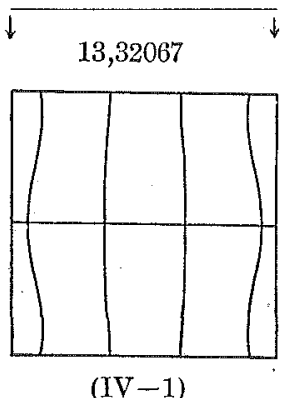

$(I V-1)$

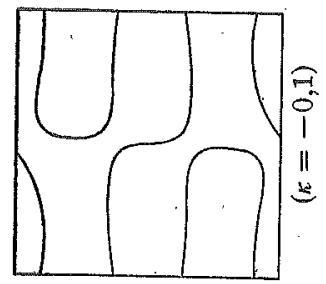

(IV-2)

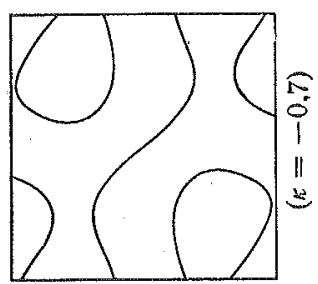

$(I V-3)$

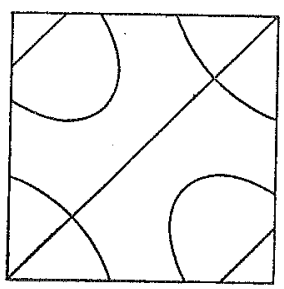

$(I V-4)$

Abb. 26. Schematische Darstellung der Knotenfiguren für die dritte Schwingungsart der quadratischen Platte mit $\nu=0,3$. 

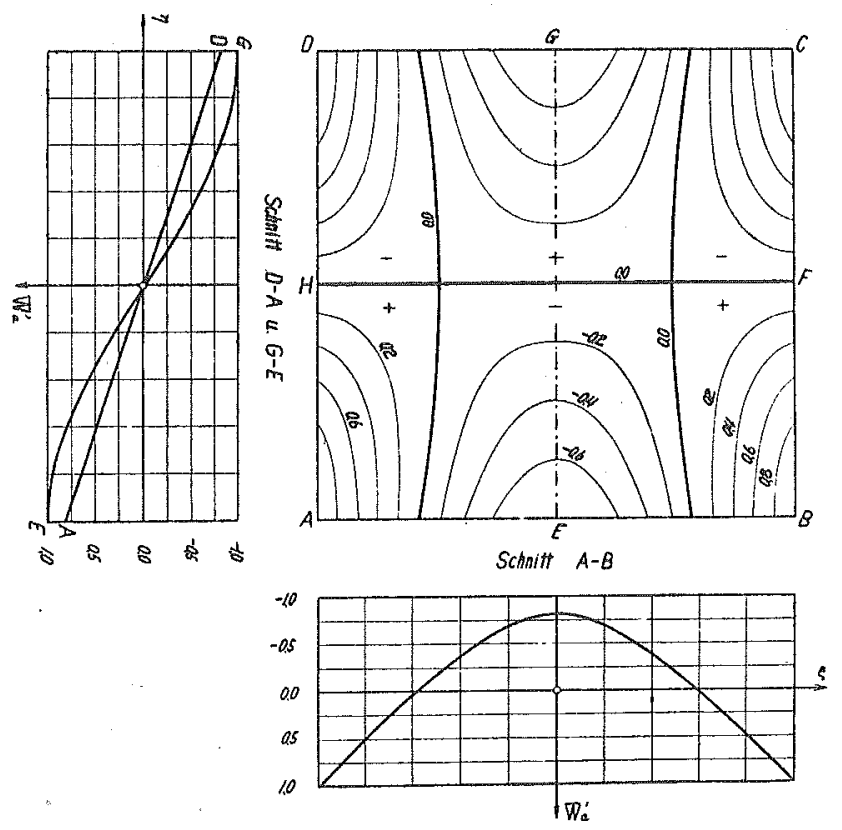

$(I-1)$

$\mu_{1}=3,52609(\kappa=0,0)$.
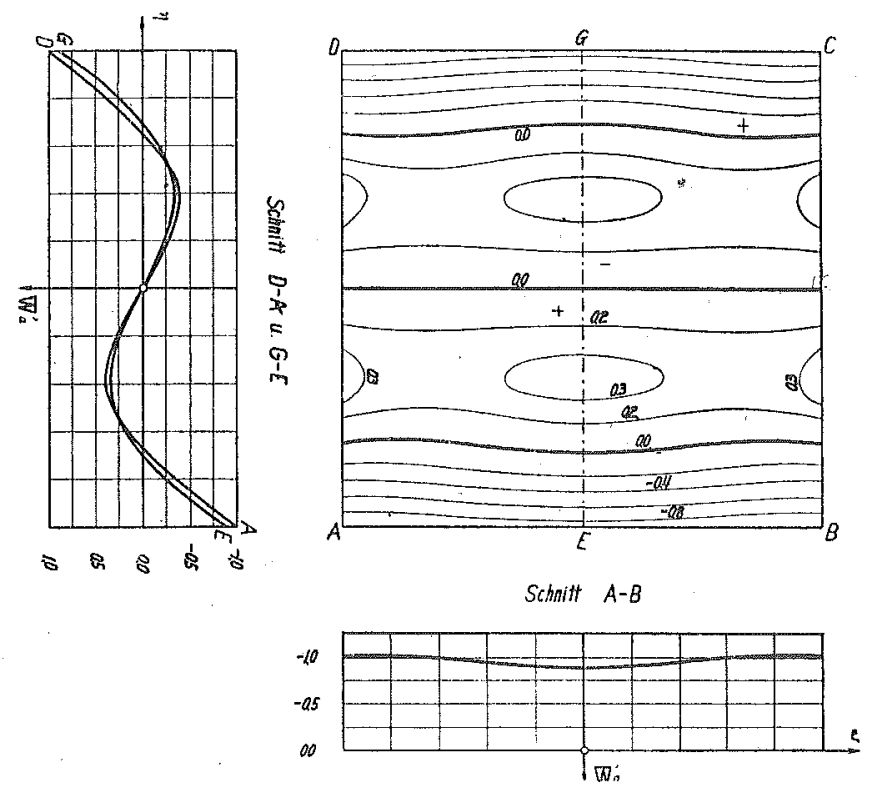

(II-I)

$\mu_{2}=6,19004(\iota=0,0)$. 

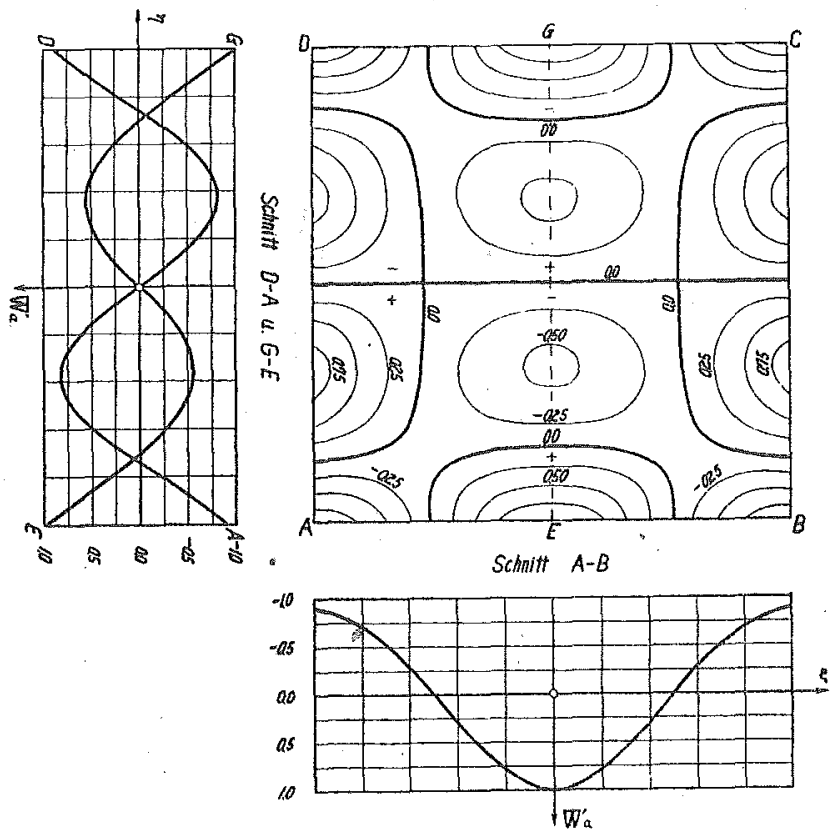

$(\mathrm{III}-1)$

$\mu_{\mathrm{i}}=10,68568(\kappa=0,0)$.
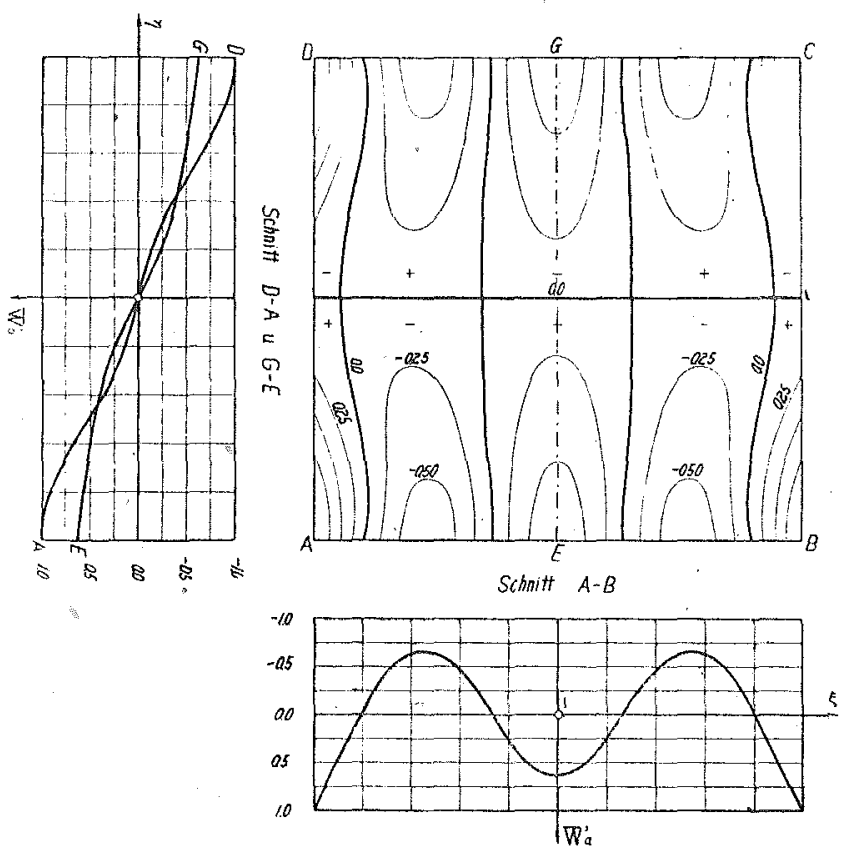

$$
(I V-1)
$$

$\mu_{i}=13,32067(\kappa=0,0)$.

Abb. 27. Schichtlinien der $\bar{w} \bar{w}_{a}^{\prime}-F l a ̈ c h e$ für die exste Familie der dritten Schwingungsart. 


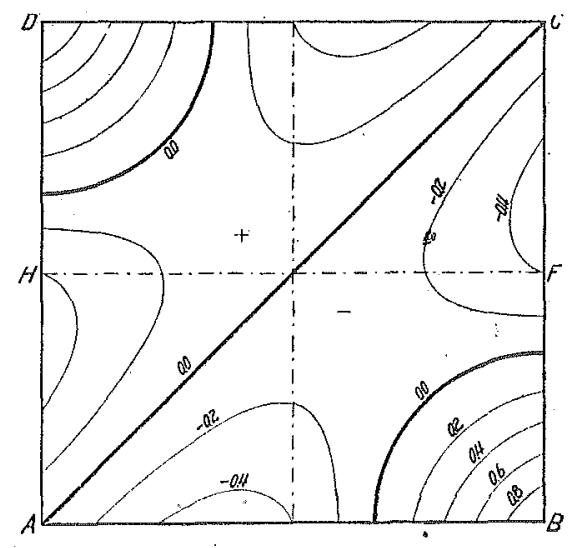

Schnitt $A-B$ u. $H-F$

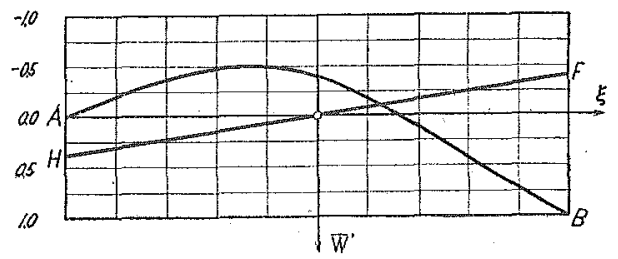

$(\mathrm{I}-4)$

$\mu_{1}=3,52609(\kappa=-1,0)$

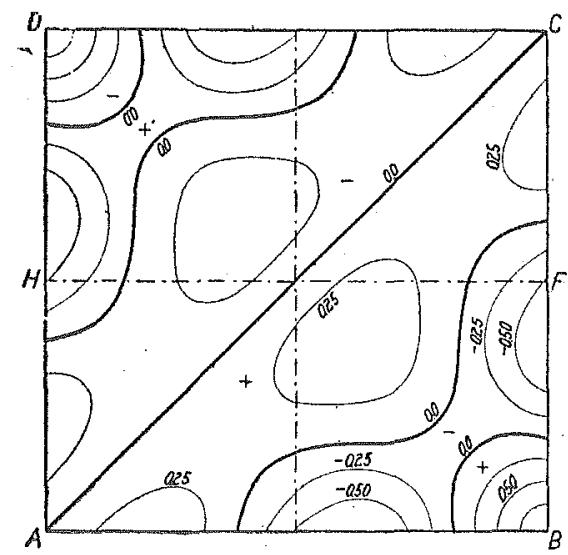

Schinith $A-B$ u. H-F

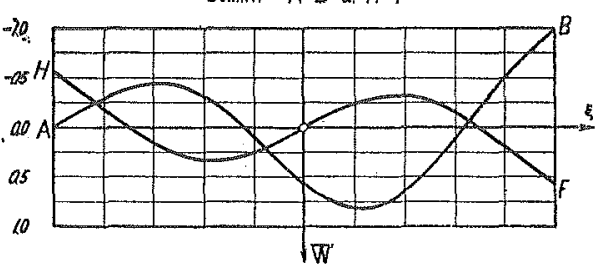

(III-4)

$\mu_{3}=10,68568(\kappa=-1,0)$.

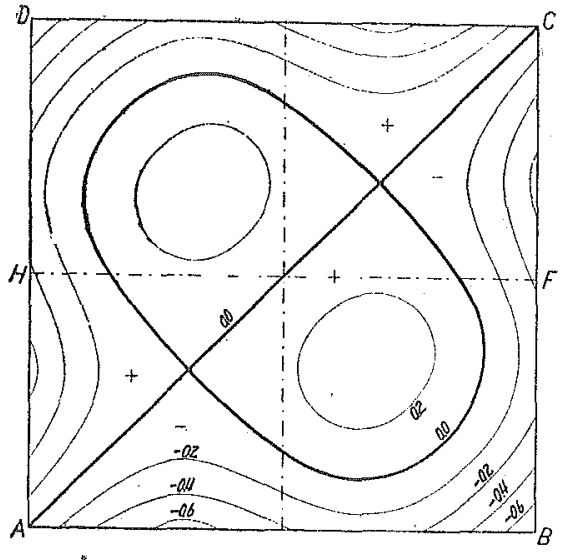

Schioitt $A-B \| H-F$

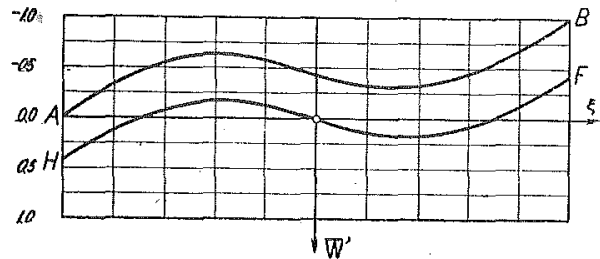

(II-4) $\mu_{2}=6,19004(=-1,0)$.

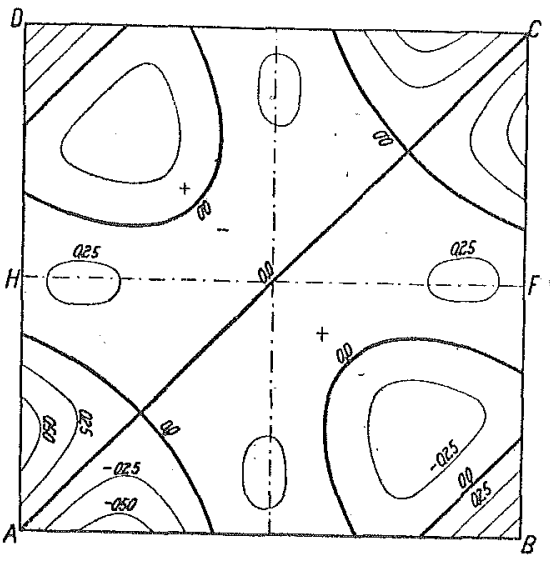

Schalit $A-B$ u. H-F

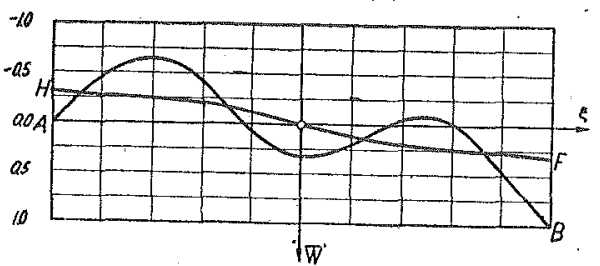

(IV-4)

$\mu_{4}=13,32067(\kappa=-1,0)$.

IAbb. 28. Schichtlinien der Fläche $\bar{w}^{\prime}=\bar{w}_{a} a^{\prime}-\bar{w}^{\prime}{ }_{b}$ für die zweite Familie der dritten Schwingungsart. 

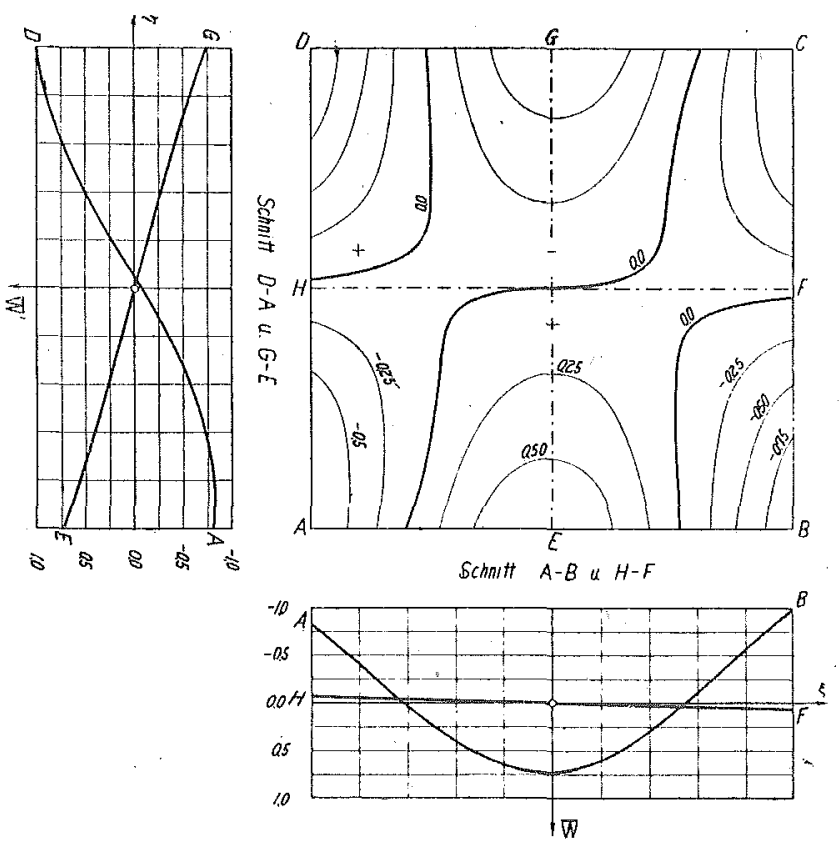

$$
(\mathrm{I}-2)
$$

$\mu_{\mathfrak{1}}=3,52609(\kappa=-0,1)$.
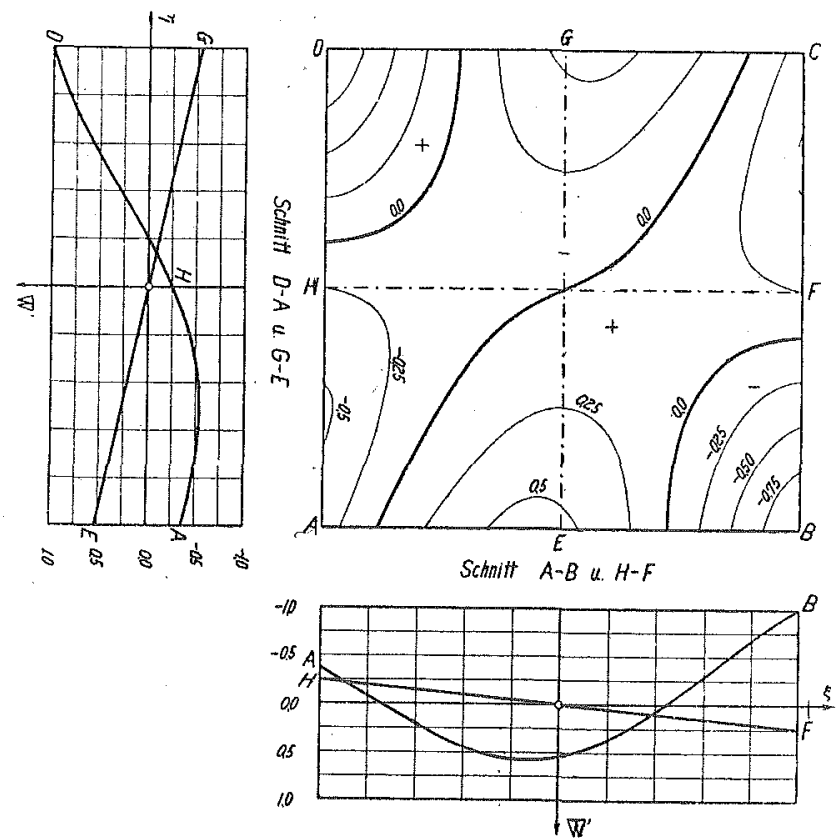

$(\mathrm{I}-3)$

$\mu_{1}=3.52609(\kappa=-0,465)$. 

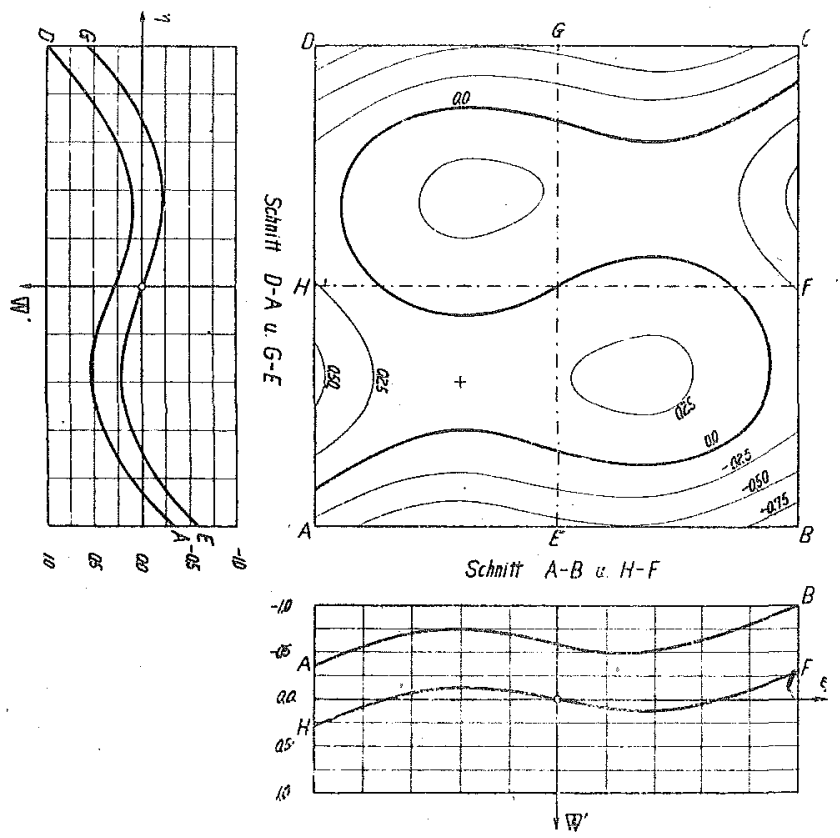

$(I I-2)$

$\mu_{\mathrm{g}}=6,19004(\kappa=-0,25)$.
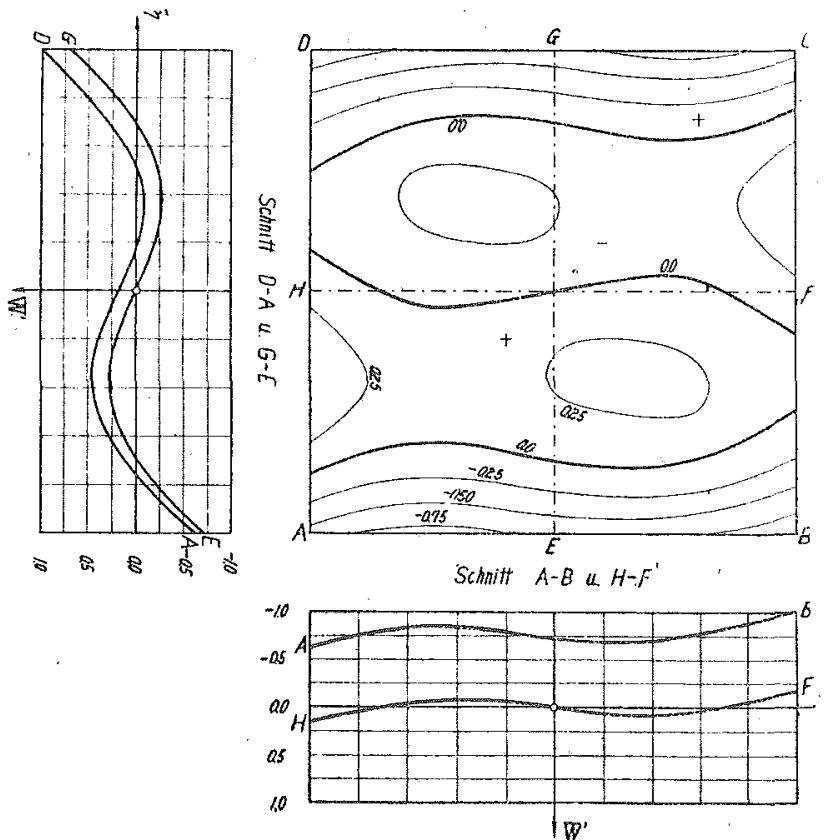

(II-3)

$\mu_{2}=6,19004(\kappa=-0,5)$. 

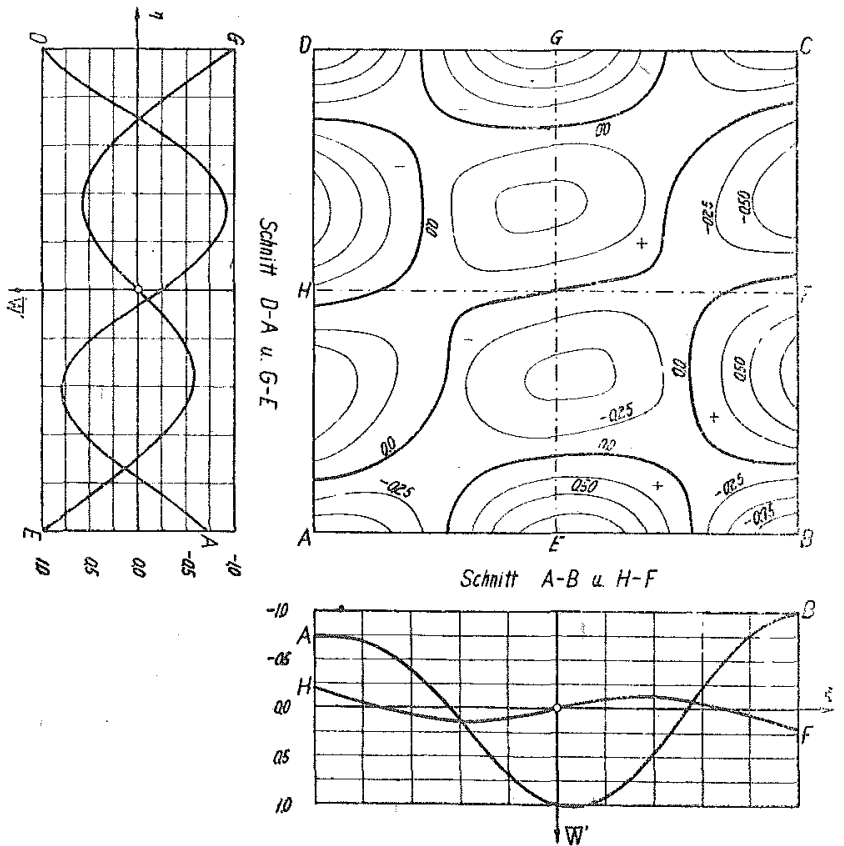

(III-2)

$\mu_{3}=10,68568(\kappa=-0,2)$.
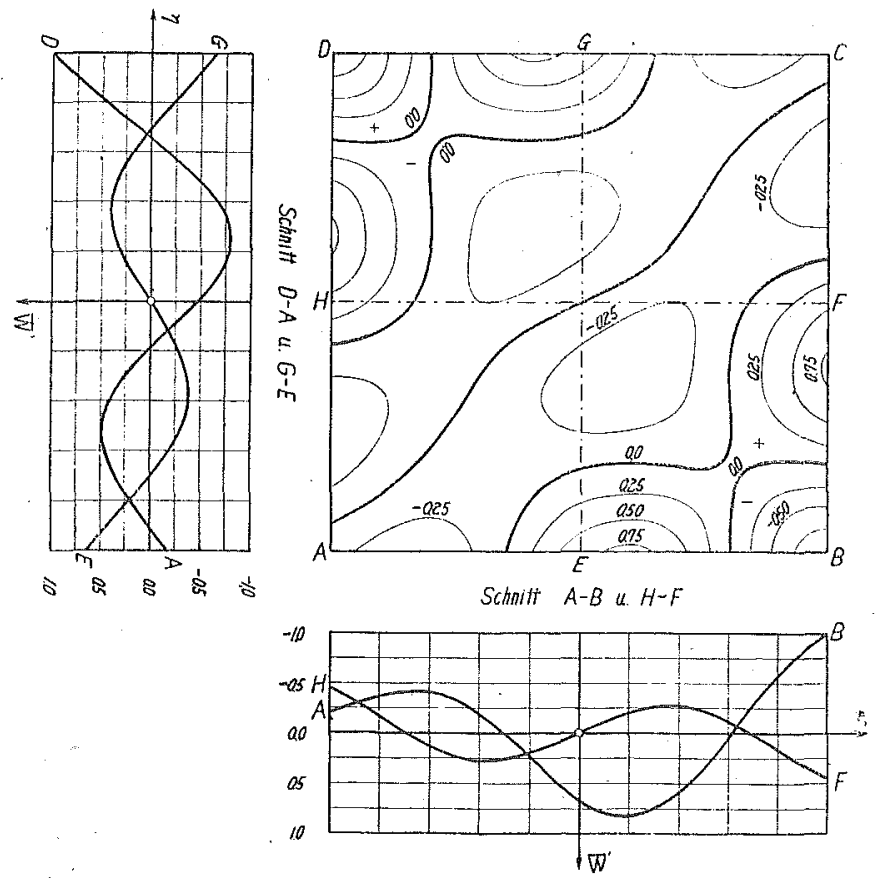

(III-3)

$\mu_{3}=10,68568(\kappa=-0,7)$. 

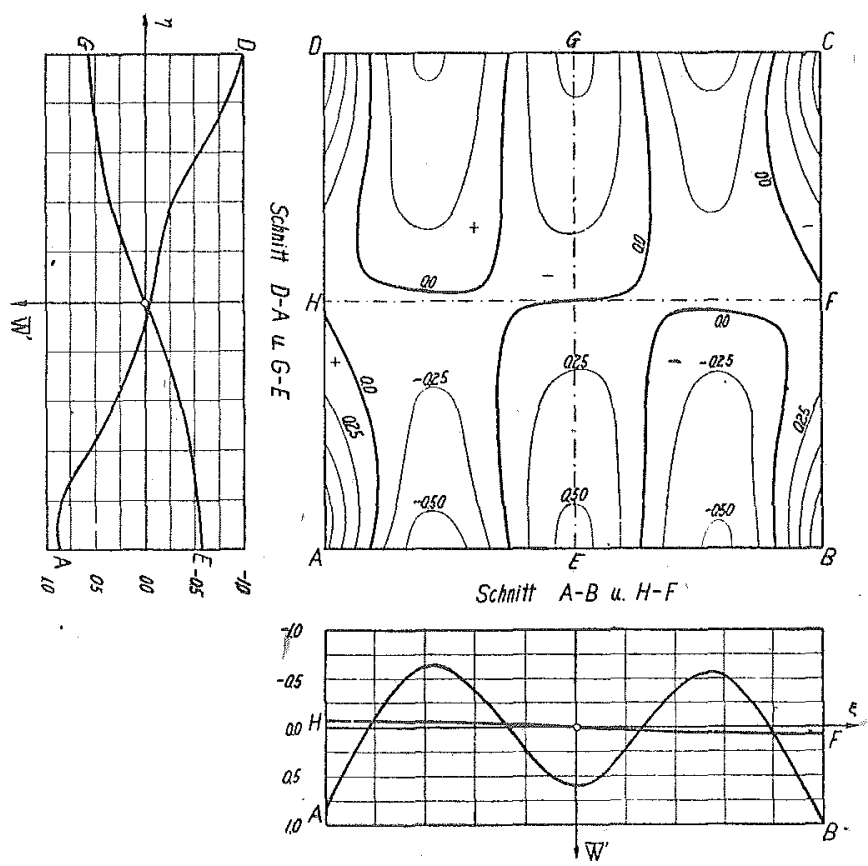

$(\mathrm{IV}-2)$

${ }^{\prime} \mu_{i}=13,32067(\kappa=-0,1)$.
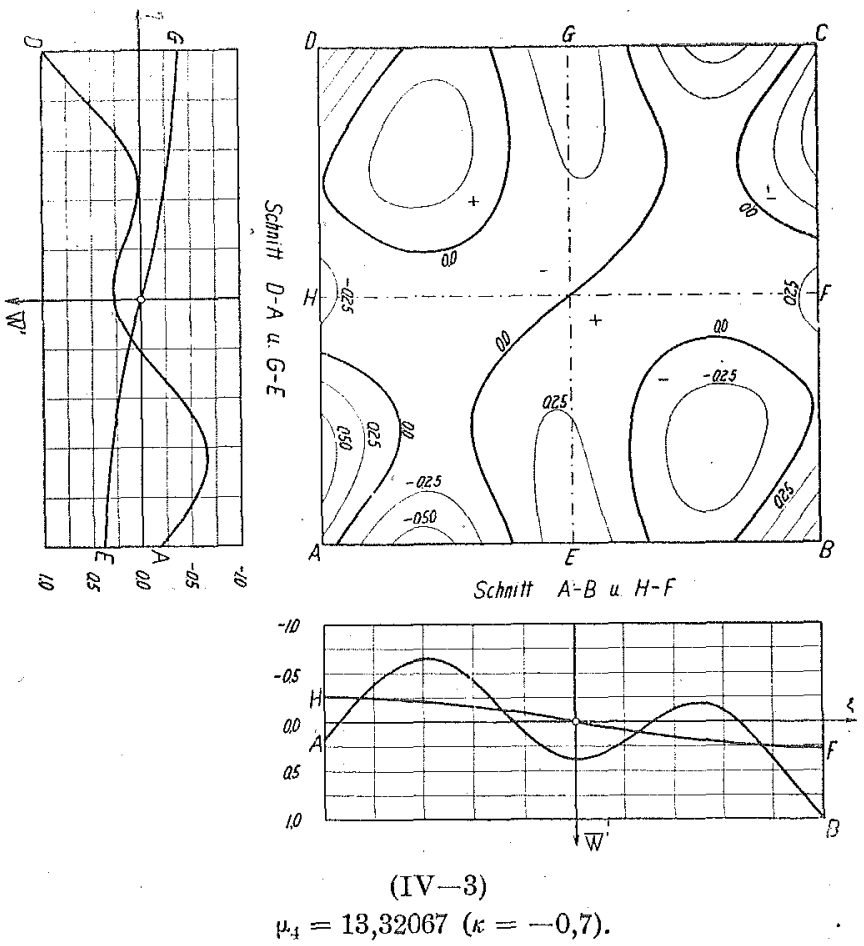

Abb. 29. Schichtlinien der Fläche $\bar{w}^{\prime}=\widetilde{u} a^{\prime}+\kappa \bar{w}_{b}^{\prime}$ für die dritte Familie der dritten Schwingungsart. 
Die jeder Wurzel $\mu$ sowie dèn verschiedenen Grössen von $\kappa$ entsprechenden Knotenfiguren für diese Familie werden in Abb. 26 mit den Zeichen (I-2), $(\mathrm{II}-2), \cdots,(\mathrm{I}-3),(\mathrm{II}-3), \cdots,(\mathrm{IV}-3)$, und die entsprechenden Schichtlinien der $\bar{w}$-Fläche in Abb. 29 dargestellt.

Trotz der natürlichen Folge ist es merkwürdig, dass die zu jeder Wurzel $\mu$ gehörende Figur für die erste Familie sich endlich mit wachsendem $|\kappa|$ nach kontinuierlichem Verlauf der zu der dritten Familie gehörendem Figur in die Figur der zweiten Familie verwandelt. Ferner ist es interessant und zugleich. auch bemerkenswert, dass der Koeffizient $\kappa$ zweckmässig so gewählt werden kann, dass die Auslenkung eines beliebig gegebenen Punktes $\left(x_{1}, y_{1}\right)$ einem beliebig gegebenen Festwert $\gamma$ gleich wird, nämlich

$$
\bar{w}_{a}^{\prime}\left(x_{1}, y_{1}\right)+\kappa \bar{w}_{b}^{\prime}\left(x_{1}, y_{1}\right)=\gamma \quad \text { oder } \quad \kappa=\frac{\gamma-\bar{u}_{a}^{\prime}\left(x_{1}, y_{1}\right)}{\bar{u}_{b}^{\prime}\left(x_{1}, y_{1}\right)} .
$$

Dies ist aber darauf beschränkt, dass die Grösse $\bar{u}_{b}^{\prime}\left(x_{1}, y_{1}\right)$ nicht gleich Null ist, oder in anderen Worten, der Punkt $\left(x_{1}, y_{1}\right)$ nicht auf den Knotenlinien $\bar{u}_{b}^{\prime}(x, y)=0$ liegt. Insbesondere hat der Nullwert von $\gamma$ die wichtigste Bedeutung, weil man hierbei die Platte so schwingen lassen kann, dass die eine ihrer Knotenlinien einen beliebig im Bereich des Quadrats ausser auf den Knotenlinien $\bar{w}_{b}^{\prime}(x, y)=0$ gewählten Punkt passiert, welche Möglichkeit man auch experimentell bestätigen kann.

Es ist leicht verständlich, dass die oben erwähnte Tatsache auch für die dritte Schwingungsart der früher in Kapitel II behandelten quadratischen Platte mit Eckstützen gilt.

\section{E. Experimentelle Untersuchung.}

Die im Vorigen von mir beschriebenen Knotenfiguren kann man grösstenteils auch in den Sandfiguren von Chladni sehen. Indessen habe ich auch gesucht, die betreffenden Sandfiguren durch gewöhnliche einfache Anordnungen zu erzeugen, um meine aus meinen theoretischen Formeln hervorgehenden Knotenfiguren mit ihnen zu vergleichen. Dazu werden quadratische Glasplatte von 2 bis $3 \mathrm{~mm}$ Dicke, 20 bis $28 \mathrm{~cm}$ Seitenlänge gebraucht, deren Zentren (oder unter Umständen die anderen Lagen) mit dem Schraubstock befestigt werden. Die Erregung der Schwingungen erfolgt wie immer durch Anstreichen mit einem Geigenbogen. Die weiteren Versuchsverfahren sind ganz wie gewöhnlich, und ein Teil der so gebildeten Sandfiguren wird in Abb. 30 bis 32 dargestellt. Die Figur mit dem Zeichen 1), 2) bzw. 3) gehört zu der ersten, zweiten bzw. dritten Familie der entsprechenden Schwingungsart.

Die Vergleichung dieser Figuren mit den theoretisch berechneten zeigt, dass einige Figuren miteinander recht gut, die anderen dagegen im grossen und gangen übereinstimmen. Es ist verständlich, dass die etwaige Abweichung hauptsächlich in 1. der Unsicherheit sowie der Undefniertheit der Befestigung der Glasplatten, 2. den verschiedenen störenden Einflüssen des Anstreichens 


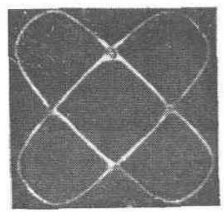

1) für $\mu_{3}$.

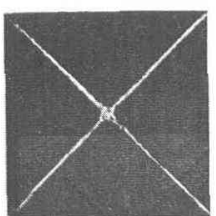

2) für $\mu_{1}$

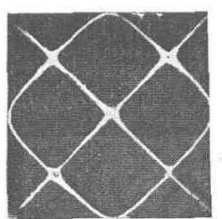

2) für $\mu_{3}$.

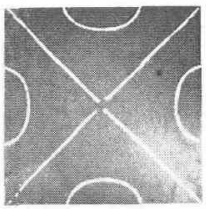

2) fïr $\mu_{4}$.

Abb. 30. Sandfiguren für die erste Schwingungsart.

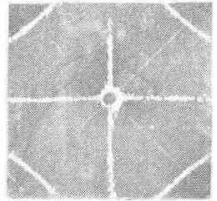

1) für $\mu_{2}$.

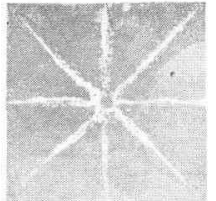

2) für $u_{1} \cdot$

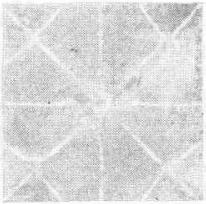

2) für $\mu_{3}$.

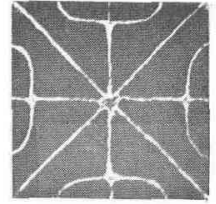

2) füir $\mu_{+}$.

Abb. 31. Sandfiguren für die zweite Schwingungsart.

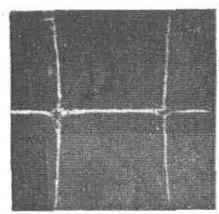

1) für $\mu_{1}$.

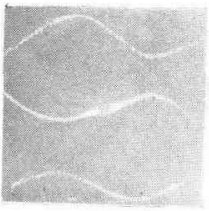

3) für ب. .

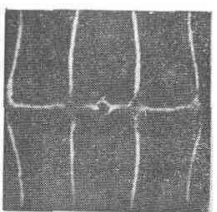

1) für $\mu_{4}$.

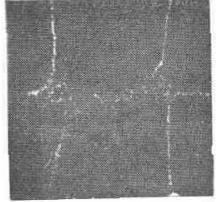

3) für $\mu_{1}$

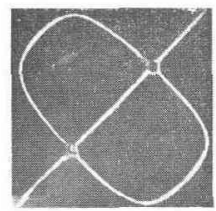

2) für $\mu_{2 .}$.

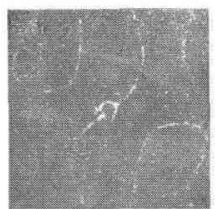

3) für $\mu_{4}$.

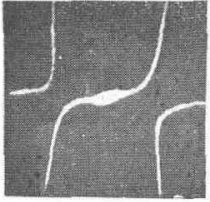

3) für $\mu_{1}$.

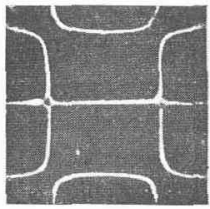

1) für $u_{3}$.

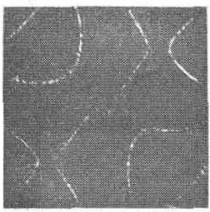

3) für $u_{4}$.

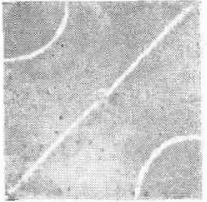

2) für $\mu_{1}$.

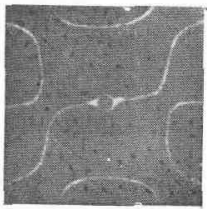

3) für $\psi_{3}$.

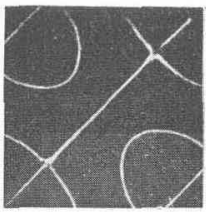

2) für $\varphi_{4}$.

Abb. 32. Sandfiguren für die dritte Schwingungsart. 
mit dem Geigenbogen, 3. der Ungenauigkeit der Abmessungen sowie der Inhomogenität der Platte, 4. der Abweichung des in den Rechnungen angenommenen Wertes von $\nu(=0,3)$ von dem wirklichen Wert von $\nu$ für die Glasplatte (etwa 0,25$)$ u.s.w. besteht.

Wie man sieht, gehören die in Abb. 32 mit den Zeichen 3) dargestellten. Figuren der dritten Familie der dritten Schwingungsart an. Da sie aber derart . erzeugt werden, dass ein aufs' Geratewohl gewählter Punkt der Plattenränder unbeweglich bleibt, so ist der. Wert von * ganz unklar. Aber' man kann den Näherungswert von $\kappa$ durch Vergleichung mit den in Abb. 26 dargestellten entsprechenden Knotenfiguren mutmasslich bestimmen. So zum Beispiel liegt es sehr nahe zu sagen, dass $\kappa$ für die in der ersten Reihe stehende Figur 3) für $\mu_{1}$ bzw. die in der untersten Reihe stehende Figur 3) für $\mu_{4}$ nur wenig von 0,0 bzw. - 1,0 abweicht, während der Wert $\kappa$ für die in der zweiten Reihe stehende 3) für $\mu_{3}$ beinahe gleich $-0,7$ sein muss. 